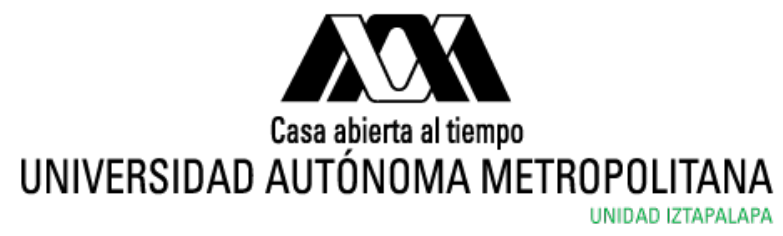

DIVISIÓN DE CIENCIAS BÁSICAS E INGENIERÍA

Departamento de Física

\title{
Partículas activas en la detección de señales débiles
}

\author{
Tesis Que PRESEnTA: \\ Ernesto Joel Espinosa Santamaria \\ PARA OBTENER EL GRADO DE: \\ MAESTRO EN CIENCIAS (FÍSICA) \\ Asesor: Dr. José Inés Jiménez Aquino
}

Sinodales:

Dr. Rosalío Fernando Rodríguez Zepeda IF-UNAM

Dr. Francisco Javier Sevilla Pérez IF-UNAM

Ciudad de México 06 diciembre de 2019, EP-001 11:00 hrs. 

Por qué recorrer este camino no hubiera sido igual de no contar con ustedes. Especial dedicación al Dr. José Inés Jiménez Aquino por su tiempo y comprensión, Alfonso y Felipa por brindarme su apoyo, Leonel quien ha sido un referente en mi vida y Samantha por acompañarme en todo momento. 


\section{Agradecimientos}

Al Dr. José Inés Jiménez Aquino por siempre tener la disponibilidad para poder llevar acabo esta tesis, gracias profesor por compartir su conocimiento..

A mis padres Alfonso y Felipa quienes siempre me brindaron su apoyo para superar todos los retos que se presentaron, gracias ustedes son mi inspiración para seguir.

A Leonel quien toda la vida ha estado para apoyarme y aconsejarme gracias hermano por siempre estar ahí.

A Iker pues durante este tiempo me enseñaste que en ocasiones uno pasa desapercibido lo sorpréndete que es la vida cotidiana.

A Samantha por acompañarme y comprenderme durante todo este trayecto, infinitas gracias por siempre brindarme tu apoyo compañía y amor.

A mis amigos Edgar, Elvis, Miguel y Benito con los que conviví durante este tiempo gracias por su apoyo, compañia y consejos, siempre pude confiar en ustedes en los momentos complicados.

A mis colegas de la UAM-I con quienes tuve la oportunidad de interactuar durante este tiempo, en especial a los compañeros del cubículo T-326 Marvin, Julio, Alberto gracias por compartir sus conocimientos y experiencias. 
Al Dr. Rosalío Fernando Rodríguez Zepeda y Dr. Francisco Javier Sevilla Pérez gracias por su tiempo y observaciones, su contribución enriqueció de gran manera el presente trabajo.

Finalmente quiero agradecer al Consejo Nacional de Ciencia y Tecnología (CONACYT) por la beca otorgado, pues me permitido llevar mis estudios.

Y por supuesto gracias a la universidad Autónoma Metropolitana porque al brindarme sus instalaciones me dio la oportunidad de adquirir el conocimiento dentro de sus aulas.

¡Que siempre tenga presente esta etapa de mi vida! 


\section{Índice general}

\begin{tabular}{ll}
\hline 1. Introducción & 2
\end{tabular}

\begin{tabular}{ll}
\hline 2. Movimiento browniano & 10
\end{tabular}

2.1. Introducción . . . . . . . . . . . . . . . . . . . . . . . 10

2.2. Ecuación de Langevin . . . . . . . . . . . . . . . . 12

2.2.1. Desplazamiento cuadrático promedio (DCP) . . . . . 13

2.3. Ecuación de Fokker-Planck . . . . . . . . . . . . 16

3. Detección de señales débiles en un Láser 18

3.1. Introducción . . . . . . . . . . . . . . . . . . . . . . . 18

3.2. Análisis cualitativo de la Teoría cuasi-determinista (QDT), un análisis lineal . . . . . . . . . . . . . . 20

3.3. El sistema láser . . . . . . . . . . . . . . . . . . . . . 22

3.3.1. Estadística de los tiempos de primer paso . . . . . . 24

3.3.2. Detección de señales ópticas débiles . . . . . . . . . 31

4. Partículas activas en la detección de señales débiles 35

4.1. Introducción. . . . . . . . . . . . . . . . . 35

4.2. La QDT y el tiempo promedio de primer paso . . . . . . . . 41

4.3. Detección de señales débiles . . . . . . . . . . . . . . . 46

$\begin{array}{ll}5 . \text { Conclusiones } & 55\end{array}$

\begin{tabular}{|ll}
\hline A. Función generadora y la estadística de los tiempos de paso. 57 &
\end{tabular}

A.1. Estadística de los tiempos de paso . . . . . . . . . . 58 
\begin{tabular}{ll}
\hline B. Matriz de rotación & 61
\end{tabular}

C. Cálculo de $\left\langle e_{i}(t)\right\rangle$ y $\left.\left\langle e_{i}(t) e_{j}\left(t^{\prime}\right)\right\rangle\right] \quad 64$

C.1. Valores promedio $\left\langle e_{i}(t)\right\rangle \ldots \ldots \ldots$. . . . . . . 64

C.2. Funciones de correlación $\left\langle e_{i}(t) e_{j}\left(t^{\prime}\right)\right\rangle \ldots \ldots \ldots$. . . . . 65

\begin{tabular}{ll}
\hline D. Cálculo de la matriz $\sigma_{i j}$ & 70
\end{tabular}

\begin{tabular}{ll}
\hline E. Escalas de tiempo & 74
\end{tabular}

\begin{tabular}{ll}
\hline Bibliografía & 78
\end{tabular} 



\section{Resumen}

De la manera más sencilla posible podemos decir que un proceso estocástico es aquel que no se puede predecir. Se mueve al azar. En la teoría de la probabilidad, es un concepto matemático que se utiliza en magnitudes aleatorias que varían con el tiempo. Los procesos estocásticos permiten tratar procesos dinámicos en los que hay cierta aleatoriedad.

El movimiento browniano es uno de los fenómenos físicos que ha sido identificado como un proceso estocástico fuera de equilibrio. Podemos decir que el MB es un movimiento en forma de Zigzag que realizan partículas pequeñas. Varios científicos contribuyeron para encontrar la explicación al MB y tiempo después Paul Langevin propone un método matemático para la solución del MB.

En 1989 distintos científicos propusieron algunos métodos para detectar señales ópticas débiles basado en la dinámica de encendido del sistema láser. Que se puede entender como un proceso de decaimiento de un estado inestable por efecto de fluctuaciones estocásticas. Para caracterizar el decaimiento de estados inestables el modelo teórico propuesto mediante la ecuación de Lagevin se conoce como teoría cuasi-determinista, que utiliza los tiempos de paso durante los cuales se lleva a cabo la relajación dinámica de la intensidad de la radiación. Estas ideas para el sistema Láser se pueden extender al proceso de relajación del estado inestable de partículas brownianas con actividad usando el mismo criterio de detección demostramos que el criterio es sensible a la señal débil a través de un parámetro BETA el cual contiene el efecto entre la señal externa y el ruido interno. Haciendo una comparación entre partículas Brownianas pasivas y activas observamos que la amplitud del campo eléctrico externo se ve incrementado en el caso de partículas brownianas activas. 


\section{Capítulo 1}

\section{Introducción}

La detección de señales débiles es un tema de gran interés en el estudio de las telecomunicaciones y en la detección super-regenerativa en los receptores de radar (Radio Deteccting and Ranging, por sus siglas en inglés) [1]. Y ¿cuál es el mecanismo básico de un radar? El principio básico es muy similar al de la reflexión de ondas sonoras. Si se emite un sonido en la dirección de un objeto que refleje el sonido (como un cañón rocoso o una cueva) es posible escuchar el eco y obtener información del objeto en cuestión. El radar emplea pulsos de energía electromagnética en una forma similar. En este caso, la energía de radiofrecuencia se transmite hacia el objeto y éste a su vez refleja dicha señal. Una pequeña fracción de la energía reflejada retorna al equipo radar que analiza dicha señal para obtener la información necesaria. En otras palabras, procesa y regenera la información recibida. Hoy en día existen algunos dispositivos de radar que funcionan de forma similar, entre los cuales podemos mencionar los receptores de radar alerta, los cuales tienen la tarea de proteger y alertar a los pilotos de una aeronave; las señales satelitales de los proveedores de internet, en este caso la información de la compañía se envía al espacio por una antena emisora hasta llegar al satélite geoestacionario que recibe la señal y la amplifica para soportar el viaje de vuelta para ser recibida y distribuida por otra antena en otra parte del mundo.

Tomando en cuenta la idea de la detección super-regenerativa en los receptores de radar, Vemuri y Roy [2] propusieron a finales de los ochenta del siglo pasado un mecanismo físico capaz de detectar señales ópticas 
débiles en un sistema Láser. Dicho mecanismo consiste en el proceso de encendido del sistema láser el cual puede actuar como un receptor superregenerativo. En esencia el proceso de encendido de un Láser consiste en la dinámica transitoria que experimenta la intensidad $I(t)$ de la luz Láser, cuando ésta evoluciona desde un valor inicial $I(0)$ hasta alcanzar el valor correspondiente de su estado estacionario $I_{s t}$, como consecuencia de las fluctuaciones estocásticas que dan cuenta de la emisión espontánea. La dinámica transitoria mencionada se lleva a cabo desde un estado inestable inicial (estado de mayor energía) al tiempo $t=0$, hasta alcanzar el estado estacionario (estado de menor energía) al tiempo $t \rightarrow \infty$. Una vez desencadenado el proceso de relajación de dicho estado inestable, inmediatamente después, al tiempo $t>0$, se hace incidir en la cavidad del Láser una señal externa débil de amplitud menor o si acaso igual que la intensidad del ruido interno, la cual habrá de detectarse en el proceso de encendido.

Desde el punto de vista teórico fueron establecidos dos criterios relacionado con la detección de señales ópticas débiles en un Láser: el criterio propuesto por Vemuri y Roy está relacionado con un parámetro adimensional conocido como Receptor de Salida (RO por sus siglas en inglés). Éste se define como el cociente $A_{e} / A_{0}$, donde $A_{e}$ representa el área bajo la curva del promedio $\langle I(t)\rangle$, cuando la dinámica está sujeta a la acción del campo eléctrico externo, y $A_{0}$ es el área bajo la curva del promedio $\langle I(t)\rangle$ en ausencia del campo eléctrico externo. Vemuri y Roy mostraron mediante cálculo numérico que el RO es muy sensible a la presencia de señales ópticas débiles.

En el mismo año de la publicación del trabajo de Vemuri y Roy, Balle et al. [3] propusieron otro criterio teórico para detectar señales ópticas débiles, basado en la estadística de los tiempos de primer paso. El criterio en este caso establece que la diferencia entre las escalas de tiempo que caracterizan el proceso de encendido en presencia y ausencia de la señal débil, debe ser mayor o igual a la máxima varianza. Este criterio permite determinar el valor crítico de un cierto parámetro que definimos como $\beta_{c}$, cuyo valor determina la intensidad de la señal débil que se debe detectar. Para valores de $\beta$ menores que $\beta_{c}$ no hay detección, mientras que para valores mayores que $\beta_{c}$ la detección se debe realizar de manera eficiente. Las escalas de tiempo antes mencionadas se conocen como tiempos promedio de primer paso (MFPT por sus siglas en inglés). Fueron 
Littler et al. [4] quienes al año siguiente llevaron a cabo el experimento y mostraron que la señal óptica débil se puede detectar mediante las mediciones de los tiempos de paso (tiempos de iniciación) del sistema láser Argón bajo la acción de una señal atenuada de un láser de He-Ne.

La explicación teórica del trabajo de Vemuri y Roy fue reportada casi dos años después por Jiménez-Aquino y Sancho [5], quienes formularon la expresión teórica del $\mathrm{RO}$ en función de la escala de tiempo conocida como tiempo de relajación no lineal (NLRT por sus siglas en inglés). Los resultados teóricos reportados en [5] fueron comparados con los resultados numéricos de Vemuri y Roy [2], y se mostró que únicamente fueron consistentes con los reportados para el sistema láser de tintura (dye láser).

Es importante destacar que las escalas de tiempo MFPT y NLRT no sólo han sido utilizados para caracterizar la dinámica transitoria o proceso de encendido del sistema láser, sino también para caracterizar la relajación dinámica de estados inestables, metaestables y marginales [6, 7], entre varias otras aplicaciones donde podemos destacar el estudio de reacciones químicas, transporte intracelular, movimientos de animales, finanzas, series de tiempo, resonancia estocástica, etc. [6, 8, 9, 10].

En el caso del decaimiento de estados inestables el modelo más conocido es la dinámica de Langevin con ruido aditivo siendo el estado inestable inicial el máximo relativo de un potencial que proporciona la fuerza determinista. El modelo teórico propuesto para caracterizar el decaimiento de estados inestables mediante la ecuación de Langevin fue establecido por primera vez por De Pasquale and Tombesi en 1979 [11]. Este modelo se conoce como teoría cuasi-determinista (QDT por sus siglas en inglés). La QDT es una buena descripción teórica debido a que proporciona el mecanismo preciso para caracterizar el proceso de decaimiento de un estado inestable. El mecanismo físico de la QDT es el siguiente: la presencia de pequeñas fluctuaciones estocásticas cambian la condición inicial alrededor del estado inestable y una vez que el sistema abandona dicho estado inicial, el proceso dinámico es dominado por la fuerza de potencial. La QDT también permite caracterizar la relajación dinámica de potenciales inestables no lineales arbitrarios los cuales son muy difíciles de tratar matemáticamente a través de la ecuación de Fokker-Planck.

Casi 20 años después de los trabajos de Vemuri y Roy [2, Balle et al. [3], fue propuesto otro mecanismo físico alternativo al sistema Láser, 
que en principio puede ser capaz de detectar amplitudes débiles de campos eléctricos. Éste consiste en la relajación dinámica del estado inestable de una partícula browniana (PB) con carga eléctrica inmersa en un baño térmico, y en presencia de campos eléctrico y magnético cruzados [12, 13]. Por curioso que parezca y a pesar de que el sistema Láser es en principio distinto al de la $\mathrm{PB}$, la dinámica que sigue la $\mathrm{PB}$ en un campo magnético constante es sorprendentemente muy similar a la dinámica del sistema Láser estudiado por los autores antes mencionados, y por esta razón la propuesta formulada por los autores tiene un sustento basado en esta analogía con el sistema Láser. La diferencia entre ambos sistemas radica en que la dinámica del sistema Láser como veremos en el contenido de esta tesis, se describe mediante una ecuación tipo Langevin para el campo eléctrico complejo, mientras que la dinámica de la $\mathrm{PB}$ con carga eléctrica se describe en términos de la ecuación de Langevin para el vector de posición. Sin embargo, a pesar de esta diferencia cuantitativa en las ecuaciones dinámicas, las trayectorias de ambos sistemas son muy parecidas y de carácter rotacional. Esto es debido a que en la ecuación tipo Langevin asociada a la dinámica de camp eléctrico del sistema Láser, contiene un matriz antisimétrica que da cuenta de la rotación del campo eléctrico (véase el capítulo 3). La ecuación de Langevin asociada a una PB en un campo magnético constante también contiene una matriz antisimétrica y por tanto la trayectoria de la partícula es igualmente rotacional.

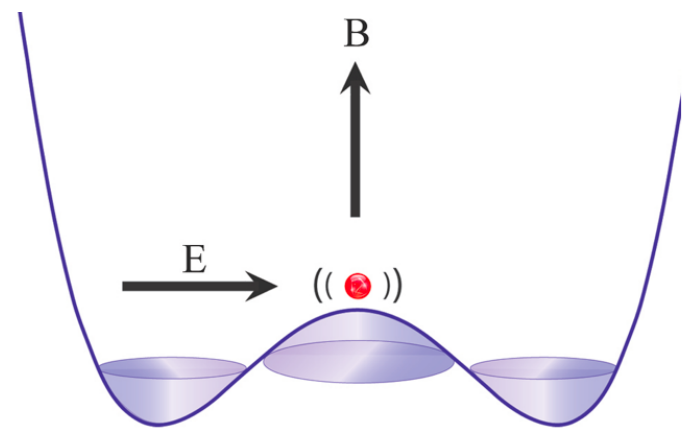

Potencial biestable $V(x)=-(a / 2) x^{2}+(b / 4) x^{4}$

Figura 1.1: Ilustración esquemática en 1D del decaimiento del estado inestable de una PB en campos eléctrico y magnético cruzados. 
En el caso de la partícula browniana, el mecanismo para el proceso de detección consiste en lo siguiente: la partícula localizada inicialmente en el estado inestable a $t=0$, experimenta el proceso de decaimiento por efecto de las fluctuaciones estocásticas, véase la Fig. 1.1. Inmediatamente después de haberse desencadenado el decaimiento del estado inestable, se hace incidir la señal externa débil acelerando el proceso de decaimiento. En principio, similar al caso del sistema Láser, este proceso debe ser capaz de detectar la señal débil incidente. La razón de esta sentencia es porque la estadística de los tiempos de paso, son también sensibles a la señal externa. La amplitud de la señal externa debe ser menor o del mismo orden que la intensidad del ruido interno.

Nuestro propósito en esta tesis consiste en estudiar el problema de la detección de señales débiles, ahora en el proceso de decaimiento del estado inestable de partículas brownianas con actividad (nadadores brownianos), inmersas en un fluido. En este caso es muy importante destacar que la materia activa es en esencia un sistema fuera de equilibrio cuyo estudio continúa siendo tema de gran interés en el terreno de los sistemas complejos, que incluyen a la física, química, biología, medicina, etc. Podemos destacar por ejemplo el estudio de sistemas biológicos como son las bacterias [14], microbios [15], motores moleculares [16, 17, 18, 19], coloides artificiales o partículas Janus [20, 21, 22, 23], entre otros [24, 25]. En el caso de los motores moleculares, conjunto de moléculas que convierten energía química en movimiento mecánico llevando a cabo tareas específicas a nivel celular, siendo éste el caso de la kinesina esencial en los procesos de mitosis, meiosis y transporte a lo largo de los axones en las neuronas. Por otra parte, a nivel macroscópico un organismo superior, por ejemplo cierto animal, se considera un sistema activo ya que toma energía del entorno al momento de alimentarse, la procesa internamente a través de complejos procesos metabólicos y finalmente manifiesta dicha energía en movimiento mecánico, lo que le permite llevar a cabo tareas esenciales para su supervivencia como lo es la misma búsqueda de alimento (forrajeo) por citar un ejemplo. La materia activa puede actuar de manera individual o formar parte de un colectivo como es el caso de una colonia de bacterias, una parvada de aves (Fig. (1.2)), cardumen de peces o una aglomeración de humanos interactuando en determinado sitio, etc. También los microrobots o micronadadores son otro tipo de partículas activas que se pueden 
construir artificialmente en el laboratorio; entre las que podemos destacar las llamadas partículas tipo Janus.

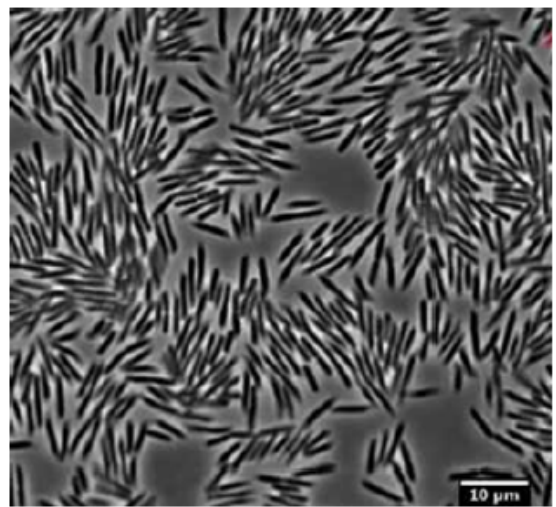

(a) Colonia de bacterias E. coli

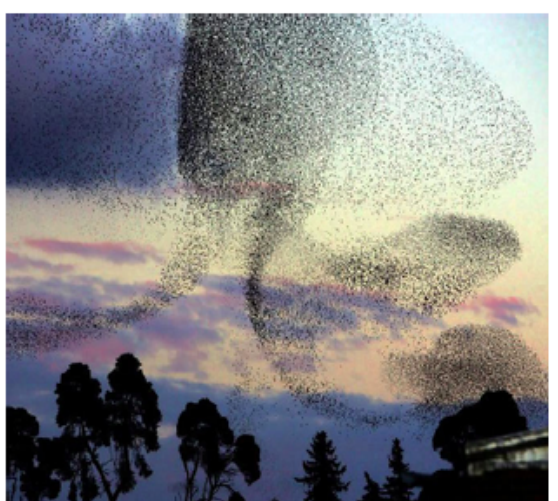

(b) Parvada de pájaros

Figura 1.2: Materia activa a nivel microscópico y macroscópico[26]

Una partícula Janus se puede transformar en una partícula browniana activa, de acuerdo con el tipo de recubrimiento específico o propiedad física que se le proporcione a su superficie. Por ejemplo, la propiedad termoforética, electroforética o alguna otra puede hacer que la partícula pasiva adquiera las características de una partícula activa. El diseño de partículas que imiten el movimiento activo así como el desarrollo de dispositivos nanotecnológicos activos, son los retos en la investigación de partículas activas. Dichos dispositivos llamados micronadadores son de suma importancia para fines terapeúticos en la medicina. Por ejemplo, pueden ser útiles para transportar ciertos fármacos por el torrente sanguíneo y aplicarlo en los sitios específicos donde se desarrolla la afectación.

Debido a las características de nuestro trabajo de tesis, es más apropiado considerar a las partículas activas artificiales y esféricas con una cierta cantidad de carga eléctrica en su superficie, como aquellas que llevarán a cabo el proceso de detección de señales eléctricas débiles, en el proceso de decaimiento del estado inestable de dichas partículas activas. Sin embargo, cabría la posibilidad de considerar otros microorganismos activos con cierta cantidad de carga eléctrica. El proceso de detección de las señales 


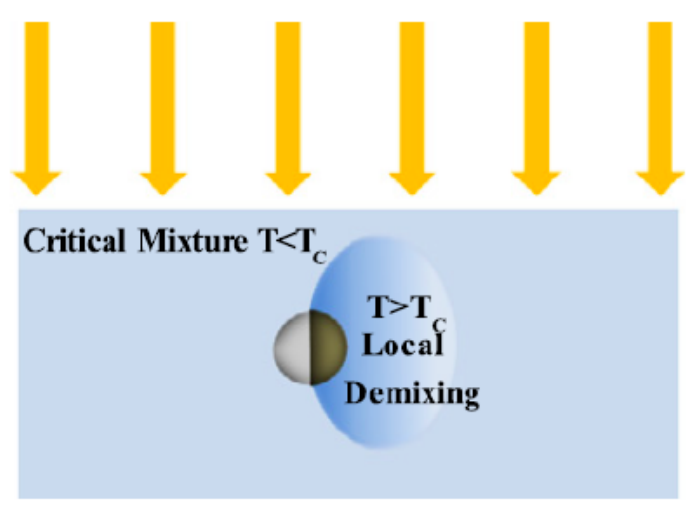

Figura 1.3: Esquema del mecanismo de autopropulsión en un micronadador: una partícula de Janus es iluminada calentando una de sus capas por encima de la temperatura crítica $T_{c}$ induciendo con ello la separación de los recubrimientos siendo este el proceso que propulsa a la partícula[27]

débiles mediante el decaimiento del estado inestable de partículas activas, se lleva acabo de la siguiente manera: al tiempo $t=0$ la partícula activa experimenta su proceso de relajación por efecto del ruido interno traslacional y rotacional. Una vez desencadenado el proceso de decaimiento, se hace incidir la señal externa débil acelerando el proceso de decaimiento del estado inestable. En nuestro problema las partículas activas están localizadas inicialmente en el estado inestable de un potencial biestable de dos dimensiones (2D). La relajación dinámica estará gobernada por la dinámica de Langevin con ruido aditivo. Supondremos que la autoactividad de las partículas brownianas introduce un grado de libertad adicional de carácter estocástico relacionado con la rotación de las partículas producido por las torcas térmicas (ruido interno rotacional). Dicho grado de libertad es el correspondiente al ángulo azimutal $\varphi(t)$ sobre el plano $x y$ perpendicular al eje $z$. En nuestra propuesta usaremos el criterio teórico de detección relacionado con la estadística de los tiempos de primer paso, para el cual será necesario caracterizar la relajación dinámica del estado inestable de la partícula activa mediante los tiempos de primer paso y su varianza. Esto se logra a través de la QDT que de nueva cuenta muestra su versatilidad 
para tal propósito.

En nuestro trabajo consideramos a los micronadadores con carga eléctrica sumergidas en un fluido simple e inactivo y nadando con un número de Reynolds pequeño que es el escenario típico para partículas activas del tamaño de micras como son, los microorganismos vivos, coloides autopropulsados, etc. Un ejemplo típico es el de la bacteria Escherichia coli, mejor conocida como E. coli, que tiene un tamaño típico entre 1-10 $\mu \mathrm{m}$, que al estar inmersa en agua a temperatura ambiente $(T=300 \mathrm{~K})$ tiene una velocidad de nado aproximado de $10 \mu \mathrm{m} / \mathrm{s}$. En este caso el número de Reynolds es del orden de $10^{-5}-10^{-4}$ [28]. En estas condiciones, las fuerzas viscosas dominan sobre las fuerzas inerciales [29] y por tanto el movimiento activo de dicha bacteria puede describirse en el contexto de la dinámica sobre-amortiguada de Langevin traslacional y rotacional.

Una vez calculada la estadística de los tiempos de primer paso, las preguntas que surgen de manera natural en el proceso de detección de señales débiles son las siguientes: ¿Cuáles son las ventajas que ofrece el estudio de la detección de señales débiles en el proceso de decaimiento del estado inestable de partículas activas, respecto al caso sin actividad? ¿El proceso de detección es más eficiente con o sin actividad? ¿Cuál es el valor de la intensidad de la señal débil que se puede detectar en presencia de la actividad? Desde luego nuestro compromiso en esta tesis es dar respuesta a las preguntas formuladas.

El presente trabajo de tesis está organizado de la siguiente manera: en el capítulo 2 hacemos un breve repaso acerca del movimiento browniano pasivo. Con el propósito de entender el proceso de detección de señales débiles en el contexto del movimiento browniano de partículas activas, en el capítulo 3, estudiamos el proceso de encendido de un sistema láser y aplicamos un criterio de detección de señales ópticas débiles, en términos de la estadística de los tiempos de primer paso. En el cuarto y último capítulo de esta tesis, proponemos el decaimiento del estado inestable de partículas activas con carga eléctrica, como otro mecanismo alternativo para detectar señales eléctricas débiles. Usamos el mismo criterio de detección formulado para el sistema láser, en términos de la estadística de los tiempos de primer paso. 


\section{Capítulo 2}

\section{Movimiento browniano}

\subsection{Introducción}

El movimiento browniano es uno de los fenómenos físicos que ha sido identificado como un proceso estocástico fuera de equilibrio. En 1827 el botánico escocés Robert Brown [30, realizó experimentalmente una investigación muy minuciosa sobre el comportamiento de una suspensión de pequeños granos de polen en una solución de agua en condiciones normales $\left(p=1 \mathrm{~atm}, T=20^{\circ} \mathrm{C}\right)$. Observó en un microscopio óptico que los granos de polen se encuentran en un estado muy animado e irregular de movimiento, el cual fue nombrado como movimiento Browniano (MB) en honor a Robert Brown. Podemos decir entonces que el MB es un movimiento en forma de zigzag que realizan partículas pequeñas, del orden de micras $\left(1\right.$ micra $\left.=10^{-6} \mathrm{~m}\right)$, que se encuentran inmersas en un fluido, ya sea líquido, gas, plasma, etc., como se muestra en la Fig. 2.1). R. Brown no fue capaz de dar una explicación de las causas de dicho movimiento, tampoco los científicos más destacados de esa época. Con el resurgimiento y desarrollo de la teoría cinética molecular de Maxwell y Boltzmann a mediados del siglo XIX, comenzaba a surgir la sospecha de que el MB era consecuencia de las colisiones entre las moléculas del fluido con dicha partícula, en el supuesto caso de que la materia estuviera constituida por átomos y moléculas, tal como lo asumieron Maxwell y Boltzmann. Sin embargo, la hipótesis de la estructura atómica y molecular de la materia no fue del todo aceptada, debido a las controversias y contradicciones que surgían al tratar 
de explicar el MB.

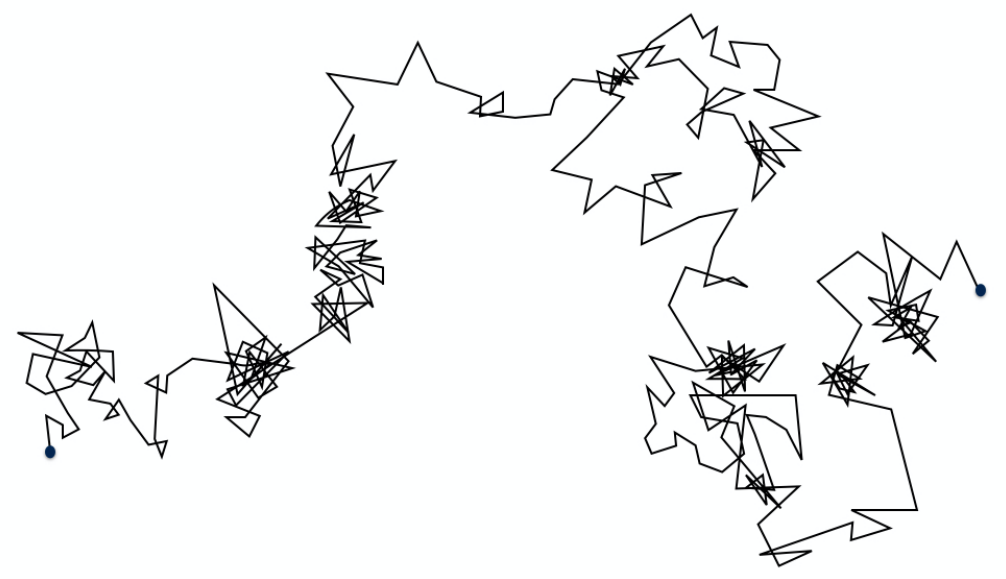

Figura 2.1: Movimiento Browniano

Todas las controversias y confusiones que se generaron para explicar las causas del MB prevalecieron hasta 1905, año en que el físico alemán Albert Einstein [31 las liquidó de forma definitiva y audaz. Un año después del trabajo de Einstein, el científico polaco Marian Soluchowski [32] publica un artículo donde de manera independiente, propone un método de solución alternativo (método del caminante al azar) al problema del MB. En 1908 el físico francés Paul Langevin [33, propone otro método muy distinto pero más simple que los dos anteriores, basado en la segunda ley de Newton. El resultado teórico propuesto por Einstein para el desplazamiento cuadrático promedio, fueron corroborados experimentalmente por el físico francés Jean Perrin y colaboradores 34 en 1909, confirmando de forma contundente la hipótesis acerca de la estructura atómica de la materia.

Una vez establecidos las estrategias de solución al problema del MB, comenzaron a desarrollarse la metodología matemática subyacente para mostrar que el esquema de Einstein y el de Langevin, son dos propuestas equivalentes de solución al problema del MB. El método fue propuesto por primera vez por Ornstein y Uhlenbeck en su artículo de 1930, donde mostraron que es posible obtener una ecuación para la distribución de probabilidad, llamada ecuación de Fokker-Planck, a partir de la ecuación de 
Langevin. En la actualidad, existe una enorme cantidad de trabajos que tienen que ver con las distintas aplicaciones del MB, y donde se muestran los distintos métodos de solución que van desde ecuaciones maestras, ecuaciones de Fokker-Planck y/o ecuaciones diferenciales estocásticas de Langevin o sus posibles generalizaciones.

Para los propósitos de esta tesis, en este capítulo vamos a establecer de manera resumida los resultados más importantes del MB mediante métodos matemáticos reportados en la literatura actual [35].

\subsection{Ecuación de Langevin}

La ecuación de Langevin asociada a una partícula Browniana inmersa en un fluido de temperatura $T$ está dada por

$$
\dot{v}=-\gamma v+\frac{1}{m} \xi(t)
$$

donde $\gamma=\alpha / m$, es el coeficiente de fricción por unidad de masa, $\alpha=6 \pi \eta a$ el coeficiente de fricción, $\eta$ la viscosidad y a el radio de la partícula. La ec. (2.1) representa una ecuación diferencial estocástica con ruido aditivo $\xi(t)$ que satisface las propiedades de un ruido blanco Gaussiano, con valor medio $\langle\xi(t)\rangle=0$ y función de correlación

$$
\left\langle\xi(t) \xi\left(t^{\prime}\right)\right\rangle=2 \lambda \delta\left(t-t^{\prime}\right)
$$

siendo $\lambda$ la intensidad del ruido. La solución formal de la ec. (2.1) está dada por

$$
v(t)=v_{0} e^{-\gamma t}+\frac{1}{m} \int_{0}^{t} e^{-\gamma\left(t-t^{\prime}\right)} \xi\left(t^{\prime}\right) d t^{\prime},
$$

donde $v_{0}=v(0)$ es la condición inicial. Usando la ec. 2.2 podemos mostrar que la función de correlación a dos tiempos está dada por

$$
\left\langle v\left(t_{1}\right) v\left(t_{2}\right)\right\rangle=v_{0}^{2} e^{-\gamma\left(t_{1}+t_{2}\right)}+\frac{2 \lambda}{m^{2}} \int_{0}^{t_{1}} \int_{0}^{t_{2}} e^{-\gamma\left(t_{1}+t_{2}-t_{1}^{\prime}-t_{2}^{\prime}\right)} \delta\left(t_{1}^{\prime}-t_{2}^{\prime}\right) d t_{1}^{\prime} d t_{2}^{\prime} .
$$

Esta doble integral se puede evaluar tomando en cuenta dos casos en el orden del tiempo, si $t_{1}>t_{2}$ o $t_{2}>t_{1}$. En primer lugar vemos que la 
función $\delta\left(t_{1}^{\prime}-t_{2}^{\prime}\right)$ limita los valores de $t_{1}^{\prime}=t_{2}^{\prime}$. Es posible demostrar que la doble integral conduce al siguiente resultado [36.

$$
\left\langle v\left(t_{1}\right) v\left(t_{2}\right)\right\rangle=v_{0}^{2} e^{-\gamma\left(t_{1}+t_{2}\right)}+\frac{\lambda}{m^{2} \gamma}\left[e^{-\gamma\left|t_{1}-t_{2}\right|}-e^{-\gamma\left(t_{1}+t_{2}\right)}\right] .
$$

Hagamos ahora un análisis a tiempos largos para los cuales $\gamma t_{1} \gg 1, \gamma t_{2} \gg$ 1. En este caso la función de correlación es independiente de la velocidad inicial y sólo depende de la diferencia de tiempos $t_{1}-t_{2}$, es decir,

$$
\left\langle v\left(t_{1}\right) v\left(t_{2}\right)\right\rangle=\frac{\lambda}{m^{2} \gamma} e^{-\gamma\left|t_{1}-t_{2}\right|} .
$$

Esto nos muestra que la velocidad es también un proceso estocástico estacionario. Si $t_{1}=t_{2}=t$, entonces es claro que en el estado estacionario

$$
\left\langle v^{2}(t)\right\rangle=\frac{\lambda}{m^{2} \gamma},
$$

luego entonces también $\left\langle v^{2}(0)\right\rangle=\frac{\lambda}{m^{2} \gamma}$. Esto nos dice que, si la velocidad tiene una distribución inicial, a saber, una distribución de Maxwell, entonces ésta se debe mantener en el estado estacionario. En dicho estado las partículas Brownianas también están en equilibrio térmico con el fluido a temperatura $T$, así que podemos aplicar el teorema de equipartición de manera tal que $(1 / 2) m\left\langle v^{2}(t)\right\rangle=(1 / 2) k_{B} T$. Usando 2.7 podemos concluir que

$$
\lambda=\alpha k_{B} T,
$$

que corresponde precisamente a la relación de fluctuación-disipación. El significado físico de esta relación es el siguiente: En el estado estacionario y en equilibrio térmico con el baño, las PBs experimentan un balance entre las fuerzas fluctuantes y las fuerzas de fricción. Estas últimas tratan de frenar el movimiento de las PBs, mientras que las primeras tienden a contrarrestar dicho amortiguamiento manteniendo en movimiento permanente a las partículas.

\subsubsection{Desplazamiento cuadrático promedio (DCP)}

Hoy en día con los avances tecnológicos en nanotecnología ya es posible controlar, manipular y monitorear moléculas mediante pinzas ópticas (rayos 
Láser); lo que permite medir la velocidad de una PB. Sin embargo, es mucho más fácil medir las funciones de correlación para la posición en vez de la función de correlación para la velocidad dada por (2.5). Si al tiempo $t=0$ la partícula está en $x_{0}$ con velocidad $v_{0}$, su DCP al tiempo $t$ será

$$
\left\langle\left(x(t)-x_{0}\right)^{2}\right\rangle=\int_{0}^{t} \int_{0}^{t}\left\langle v\left(t_{1}\right) v\left(t_{2}\right)\right\rangle d t_{1} d t_{2},
$$

donde el integrando está dado por (2.5). Sustituyendo cada término en (2.9), vemos que

$$
\begin{gathered}
\int_{0}^{t} \int_{0}^{t} e^{-\gamma\left(t_{1}+t_{2}\right)} d t_{1} d t_{2}=\frac{1}{\gamma^{2}}\left(1-e^{-\gamma t}\right)^{2} \\
\int_{0}^{t} \int_{0}^{t} e^{-\gamma\left|t_{1}-t_{2}\right|} d t_{1} d t_{2}=2 \int_{0}^{t} d t_{1} \int_{0}^{t_{1}} e^{-\gamma\left(t_{1}-t_{2}\right)} d t_{2}=\frac{2}{\gamma} t-\frac{2}{\gamma^{2}}\left(1-e^{-\gamma t}\right),
\end{gathered}
$$

en consecuencia

$$
\left\langle\left(x(t)-x_{0}\right)^{2}\right\rangle=\frac{1}{\gamma^{2}}\left(v_{0}^{2}-\frac{\lambda}{m^{2} \gamma}\right)\left(1-e^{-\gamma t}\right)^{2}+2 \frac{\lambda}{m^{2} \gamma^{2}} t-2 \frac{\lambda}{m^{2} \gamma^{3}}\left(1-e^{-\gamma t}\right) .
$$

Como podemos observar, hemos obtenido la expresión exacta del DCP de la PB para todo tiempo $t \geq 0$. En la solución (2.3), hemos supuesto que la velocidad inicial $v_{0}$ es fija, sin embargo, podría estar inicialmente distribuida por una Maxwelliana con valor medio cero y varianza $\left\langle v_{0}^{2}\right\rangle=$ $\lambda / m^{2} \gamma$, esto implica que el primer término de 2.12) es cero. En este caso el DCP será

$$
\left\langle\left(x(t)-x_{0}\right)^{2}\right\rangle=2 \frac{\lambda}{m^{2} \gamma^{2}} t-2 \frac{\lambda}{m^{2} \gamma^{3}}\left(1-e^{-t / \tau_{r}}\right),
$$

donde $\tau_{r}=1 / \gamma$. En la Fig. 2.2 se muestra el comportamiento del DCP 2.13 como función del tiempo. La gráfica muestra dos regiones representados por ON y NM, las cuales corresponden a los siguientes casos límite del tiempo de observación comparados con el tiempo de relajación $\tau_{r}$.

(i). Tiempos cortos, tales que $\gamma t \ll 1$ o $t \ll \tau_{r}$. Mediante la expansión en serie de Taylor de la exponencial podemos verificar fácilmente que $\left\langle\left(x(t)-x_{0}\right)^{2}\right\rangle=\left(\lambda / m^{2} \gamma\right) t^{2}$. 


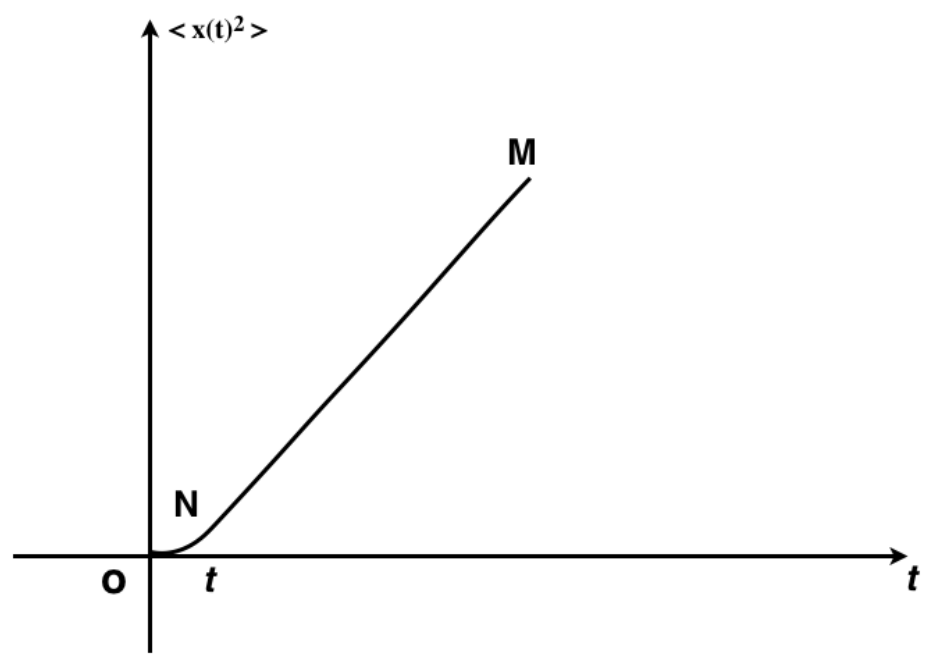

Figura 2.2: Desplazamiento Cuadrático Promedio (2.13) como función del tiempo, tal que $\left\langle\left(x(t)-x_{0}\right)^{2}\right\rangle \equiv\left\langle x^{2}\right\rangle$.

(ii). Tiempos largos, tales $\gamma t \gg 1$ o $t \gg \tau_{r}$. En este caso se puede verificar que $\left(\lambda / m^{2} \gamma^{2}\right) t \gg\left(\lambda / m^{2} \gamma^{3}\right)$, y por tanto

$$
\left\langle\left(x(t)-x_{0}\right)^{2}\right\rangle=2\left(\frac{\lambda}{m^{2} \gamma^{2}}\right) t=2\left(\frac{k_{B} T}{\alpha}\right)=2 D t,
$$

donde hemos usado la relación de fluctuación-disipación (2.8) y, $D=$ $k_{B} T / \alpha$, es precisamente el coeficiente de difusión de Einstein.

Estos resultados se pueden interpretar de la siguiente manera: para tiempos cortos el DCP es proporcional a $t^{2} \mathrm{y}$, de acuerdo con la mecánica Newtoniana se sabe que el desplazamiento de una partícula libre es también proporcional a $t^{2}$ (curva ON de la Fig. (2.2)). Por lo tanto, para tiempos cortos podemos concluir que la $\mathrm{PB}$ se comporta como una partícula libre que todavía no siente la presencia del fluido en la que está inmersa. A este régimen de tiempos cortos se le conoce en la literatura como régimen balístico. Para tiempos largos $t \gg \tau$ el DCP es proporcional a $t$ (línea recta NM de la Fig. (2.2) y, de acuerdo con la hidrodinámica clásica un comportamiento rectilíneo de ese tipo corresponde a la difusión de una 
partícula en un fluido. Podemos decir entonces que para tiempos largos la PB experimenta un proceso de difusión debido a las constantes colisiones con las moléculas del fluido, es decir, la partícula siente la presencia del fluido en la que está inmersa. A este régimen de tiempos se le conoce como régimen difusivo o régimen sobre-amortiguado, y como veremos en seguida, la terminología de sobre-amortiguado significa que la fuerza de fricción es dominante con respecto a la fuerza inercial, y por tanto la ecuación de Langevin (2.1) en este caso se escribe como

$$
\dot{x}=\xi(t) / \alpha .
$$

Así, el DCP también se obtiene de la siguiente doble integración

$$
\begin{aligned}
\left\langle\left(x(t)-x_{0}\right)^{2}\right\rangle & =\frac{2 \lambda}{\alpha^{2}} \int_{0}^{t} \int_{0}^{t}\left\langle\xi\left(t_{1}\right) \xi\left(t_{2}\right)\right\rangle d t_{1} d t_{2} \\
& =2 \frac{\lambda}{\alpha^{2}} t=2 D t
\end{aligned}
$$

que corresponde precisamente al resultado obtenido anteriormente en el límite de tiempos largos. Así que el régimen difusivo es equivalente el régimen sobre-amortiguado.

\subsection{Ecuación de Fokker-Planck}

En general y en caso de un proceso estocástico Markoviano, dada una ecuación diferencial estocástica es posible obtener su ecuación de FokkerPlanck (EFP) asociada, para la densidad de probabilidad. En el caso de la ecuación de Langevin dada por la ec. (2.1), su correspondiente EFP para la densidad de probabilidad condicional $P\left(v, t \mid v_{0}\right)$ está dada por

$$
\frac{\partial P\left(v, t \mid v_{0}\right)}{\partial t}=\gamma \frac{\partial v P\left(v, t \mid v_{0}\right)}{\partial v}+\frac{\lambda}{m^{2}} \frac{\partial^{2} P\left(v, t \mid v_{0}\right)}{\partial v^{2}},
$$

sujeta a la condición inicial $P\left(v, 0 \mid v_{0}\right)=\delta\left(v-v_{0}\right)$. Su solución para todo tiempo $t>0$ es entonces

$$
P\left(v, t \mid v_{0}\right)=\sqrt{\frac{m}{2 \pi k_{B} T\left(1-e^{-2 \gamma t}\right)}} \exp \left[\frac{m\left(v-v_{0} e^{-\gamma t}\right)^{2}}{2 \pi k_{B} T\left(1-e^{-2 \gamma t}\right)}\right] .
$$


En el límite de $t \rightarrow \infty$ esta distribución de probabilidad converge a la distribución de equilibrio de Maxwell, $P_{s t}(v)=\sqrt{m / 2 \pi k_{B} T} \exp \left(-m v^{2} / 2 k_{B} T\right)$, tal como era de esperarse. Podemos corroborar que en el estado de equilibrio estacionario $\left\langle v^{2}\right\rangle=k_{B} T / m$, que es exactamente la misma que la ec. 2.7) obtenida a través de la solución de la ecuación de Langevin (2.1).

También en el régimen sobre-amortiguado, la ecuación de Langevin 2.15 tiene asociada su correspondiente EFP para la densidad de probabilidad condicional $P\left(x, t \mid x_{0}\right)$. En este caso dicha EFP es la ecuación de difusión obtenida por Einstein en 1905, es decir

$$
\frac{\partial P\left(x, t \mid x_{0}\right)}{\partial t}=D \frac{\partial^{2} P\left(x, t \mid x_{0}\right)}{\partial x^{2}},
$$

sujeta a la condición inicial $P\left(x, 0 \mid x_{0}\right)=\delta\left(x-x_{0}\right)$ y cuya solución está dada por

$$
P\left(x, t \mid x_{0}\right)=\frac{1}{\sqrt{4 \pi D t}} \exp \left(-\frac{\left(x-x_{0}\right)^{2}}{4 D t}\right) .
$$

Es claro que en este caso el proceso estocástico 2.15 no es estacionario. También a partir de 2.20 se concluye que el DCP $\left\langle\left(x(t)-x_{0}\right)^{2}\right\rangle=2 D t$.

Podemos concluir este capítulo haciendo las siguientes anotaciones: la ecuación de Langevin (2.1) junto con la propiedades estadísticas de ruido blanco gaussiano, describen el proceso conocido como proceso de Ornstein-Uhlenbeck, y por tales motivos, se dice que es un proceso estocástico Markoviano, Gaussiano y estacionario. Fue estudiada de forma exhaustiva precisamente por Ornstein y Uhlenbeck en 1930 [37. La ec. 2.15] junto con las propiedades estadísticas de ruido blanco gaussiano, describen el proceso conocido como proceso de Wiener, el cual es también Markoviano y gaussiano, pero no estacionario. Esta ecuación se obtiene cuando la fuerza de fricción es mayor que la fuerza inercial. A partir de la ec. (2.15) se obtiene de manera inmediata el DCP dado por 2.16). Esta aproximación de fricción dominante se conoce también como régimen sobre-amortiguado mencionado anteriormente. En conclusión, el estudio del MB mediante la ecuación de Langevin o de Fokker-Planck son totalmente equivalentes. La elección de una o la otra descripción depende del grado de dificultad del problema a estudiar, o de la estrategia matemática requerida. 


\section{Capítulo 3}

\section{Detección de señales débiles en un Láser}

\subsection{Introducción}

En este capítulo vamos a estudiar un criterio teórico basado en la estadística de los tiempos de primer paso, propuesto por Balle et al. [3], para detectar señales débiles en el proceso de encendido de un sistema Láser. Para tal propósito, primeramente, debemos aclarar qué se entiende como tiempos de primer paso. El tiempo de primer paso, se define como el tiempo requerido por un proceso estocástico o trayectoria estocástica, para alcanzar por primera vez un cierto valor previamente establecido, comenzando desde un valor inicial; por ejemplo el valor de cierta frontera que delimita al sistema, o el valor de una barrera de potencial, etc. Este tiempo de paso es aleatorio y para poder describir al sistema estocástico en términos de esta cantidad es necesario calcular la estadística de dichos tiempos de paso. Aquí usaremos este concepto para caracterizar el proceso de encendido de un sistema Láser, también conocido como la dinámica transitoria de un sistema Láser o equivalentemente como el decaimiento del estado inestable de dicho sistema, debido a la emisión espontánea. Hemos comentado que el proceso de encendido consiste esencialmente en un proceso durante el cual la intensidad $I(t)$ de la luz Láser evoluciona desde un valor inicial $I(0) \equiv I_{0}$, al tiempo $t=0$, hasta alcanzar el valor de su estado estacionario 
correspondiente $I(\infty) \equiv I_{s t}$, cuando $t \rightarrow \infty$, como consecuencia del proceso de emisión espontánea.

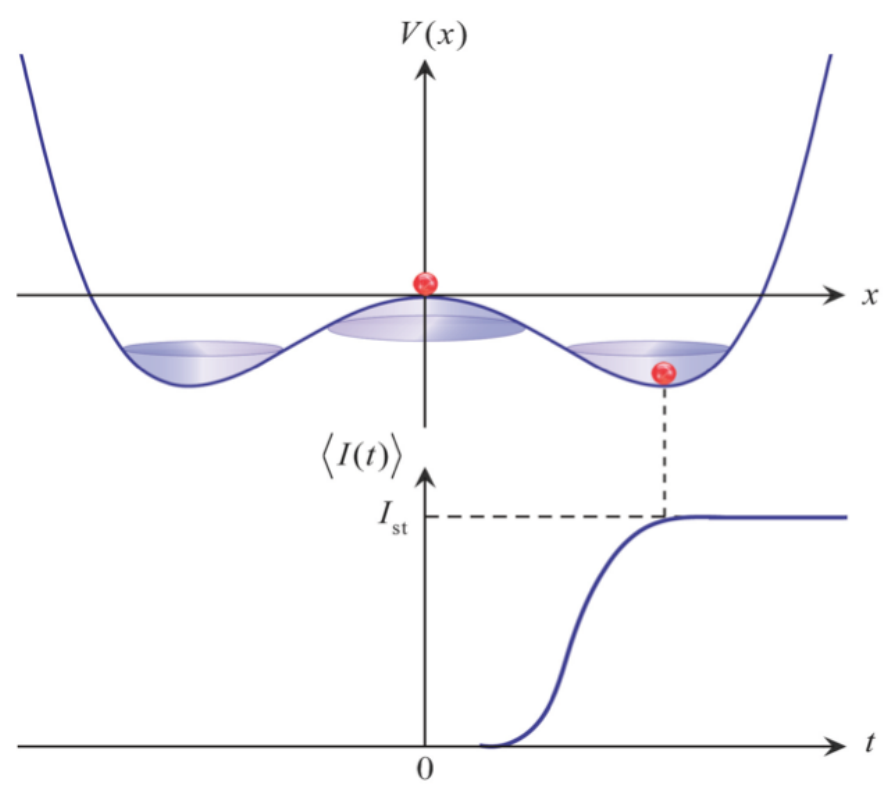

Figura 3.1: Se ilustra el proceso de encendido del sistema Láser. En la figura superior se ilustra el proceso de decaimiento de un estado inestable.

El proceso de encendido se puede caracterizar a través de la estadística de los tiempos de primer paso y dicha estadística fue propuesto como un criterio teórico para detectar señales ópticas débiles en el sistema Láser [3]. El cálculo de la estadística de los tiempos de paso fue formulada en términos de la QDT, la cual a su vez ha sido formulada en términos de la dinámica de Langevin en la aproximación lineal. La QDT ha demostrado ser una excelente teoría para caracterizar el decaimiento de un estado inestable ya que proporciona la descripción precisa del mecanismo responsable del decaimiento de dicho estado. El mecanismo ocurre de la siguiente manera: (i) fluctuaciones estocásticas pequeñas son las responsables del decaimiento del estado inestable inicial, y (ii) una vez que el sistema abandona dicho estado, la dinámica es prácticamente determinista dominada por la fuerza 
del potencial.

Debemos mencionar que el criterio antes mencionado, también se puede aplicar si la caracterización dinámica se realiza a través de los tiempos de paso, tomando en cuenta las contribuciones no lineales de la dinámica de Langevin del sistema Láser. Sin embargo, cualquiera que sea la dinámica, lineal o no lineal, el criterio conduce al mismo valor crítico del parámetro requerido para cuantificar la detección de la señal débil. Por esta razón y de acuerdo con el criterio, es suficiente con caracterizar el proceso de decaimiento del estado inestable del sistema Láser en el régimen lineal. Existe otro criterio para detectar señales débiles en un sistema Láser, el cual fue propuesto por Vemuri y Roy [2] en términos de un parámetro adimensional conocido como el receptor de salida (Received-Output (RO), por sus siglas en inglés). En el año de 1971, el RO se pudo expresar en términos del tiempo de relajación no lineal (NLRT) [5], el cual caracteriza el decaimiento del estado inestable tomando en cuenta la relajación del sistema cuando éste alcanza el valor de su estado estacionario $I_{s t}$. En este capítulo sólo estudiaremos el criterio de la estadística de los tiempos de paso aplicado en el régimen lineal y es el mismo que aplicaremos en el capítulo 4, para el caso de partículas activas.

Para entender mejor la QDT en la caracterización del decaimiento de un estado inestable, en el siguiente apartado hacemos un análisis cualitativo de dicha teoría mediante la dinámica de Langevin de una partícula browniana localizada en el máximo de un potencial inestable al tiempo $t=0$

\subsection{Análisis cualitativo de la Teoría cuasi-determinista (QDT), un análisis lineal}

Consideremos el caso de una partícula browniana inmersa en un fluido y localizada inicialmente en el estado inestable de un potencial de la forma $V(x)=-(a / 2) x^{2}$, el cual corresponde a la aproximación lineal del potencial biestable de la Fig. 3.2. Supondremos también que la dinámica que sigue la partícula está restringida en el intervalo $-R \leq x \leq R$, donde $R$ representa la barrera absorbente del potencial.

Inicialmente, la partícula localizada en el estado inestable, está sometida a fluctuaciones estocásticas por lo que la dinámica satisface la simple 
3.2. Análisis cualitativo de la Teoría cuasi-determinista (QDT), un análisis lineal

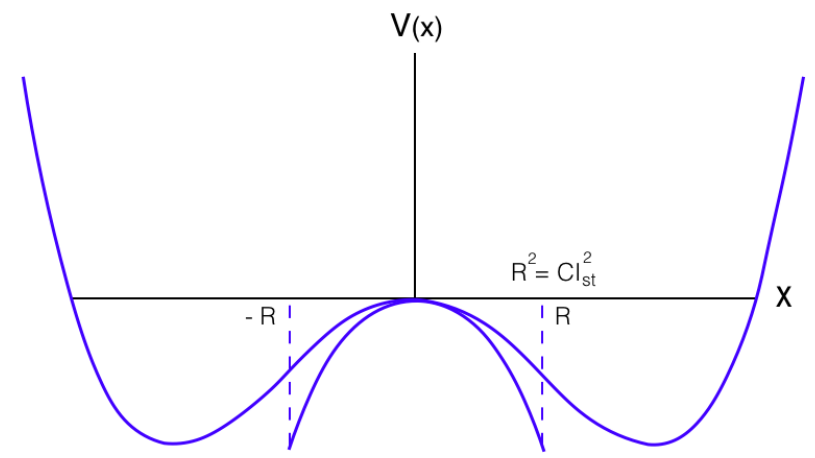

Figura 3.2: Aproximación lineal de un potencial biestable.

ecuación diferencial estocástica lineal, dada por

$$
\dot{x}=a x+\xi(t),
$$

donde $\xi(t)$ es un ruido blanco Gaussiano con valor medio $\langle\xi(t)\rangle=0 \mathrm{y}$ función de correlación $\left\langle\xi(t) \xi\left(t^{\prime}\right)\right\rangle=2 \lambda \delta\left(t-t^{\prime}\right)$, siendo $\lambda$ la intensidad del ruido. La solución formal de la ecuación anterior se puede escribir como

$$
x(t)=x_{0} e^{a t}+e^{a t} \int_{0}^{t} e^{-a s} \xi(s) d s .
$$

o bien

$$
x(t)=h(t) e^{a t}, \quad h(t)=x_{0}+\int_{0}^{t} e^{-a s} \xi(s) d s .
$$

Por simplicidad suponemos una condición inicial fija tal que $x_{0}=0$. En este caso es fácil mostrar que

$$
\left\langle h^{2}(t)\right\rangle=\frac{\lambda}{a}\left(1-e^{-2 a t}\right),
$$

y por lo tanto el DCP será

$$
\left\langle x^{2}(t)\right\rangle=\frac{\lambda}{a}\left(e^{2 a t}-1\right) .
$$

De esta simple expresión podemos estudiar también los dos casos límites para el tiempo, el límite de tiempos cortos y el límite de tiempos largos 
- Tiempos cortos tales que $t \ll 1 / 2 a$. En este caso podemos mostrar fácilmente que el DCP está dado por $\left\langle x^{2}(t)\right\rangle=2 \lambda t$.

- Tiempos largos tales que $t \gg 1 / 2 a$. En esta aproximación el DCP es ahora $\left\langle x^{2}(t)\right\rangle=(\lambda / a) e^{2 a t}$.

Estos resultados nos muestran claramente lo siguiente: el comienzo del decaimiento del estado inestable de la partícula ocurre en el régimen de tiempos cortos, tal como podemos observar, el DCP es lineal en el tiempo lo que significa que la partícula experimenta un proceso difusivo alrededor del estado inestable y como consecuencia está sometida a las constantes colisiones con las moléculas del fluido o baño térmico en la que está inmersa.

En el límite de tiempos largos, una vez que la partícula abandona el estado inestable por efecto de pequeñas fluctuaciones ( $\lambda$ pequeña), el DCP es dominado por el factor determinista $e^{2 a t}, \mathrm{y}$ como consecuencia la dinámica de la partícula es dominada por la fuerza del potencial, es decir, es prácticamente determinista. En este caso el tiempo requerido por la partícula en alcanzar la barrera del potencial $\pm R$ será $t=(1 / 2 a) \ln \left(a R^{2} / \lambda\right)$. Esta escala de tiempo característico del decaimiento de todo estado inestable, depende de los parámetros del sistema y de las fluctuaciones y esencialmente va como $t \sim \ln \left(R^{2} / \lambda\right)$, siendo $R^{2}$ el valor de la barrera del potencial y $\lambda$ la intensidad del ruido interno. A este régimen de aproximación de tiempos largos en el decaimiento de estados inestables se le conoce como la aproximación cuasi-determinista. En conclusión esta escala de tiempo es el característico asociado al decaimiento de todo estado inestable inducido por fluctuaciones estocásticas. Después de realizar un análisis cualitativo de la QDT, estamos listos para estudiar el criterio propuesto por Balle et al. para detectar señales ópticas débiles en el proceso de encendido de un sistema Láser.

\subsection{El sistema láser}

Las características básicas del sistema Láser más elemental consiste de un sistema de $N$ átomos que poseen dos niveles de energía. El sistema se encuentra en una cavidad sobre la cual se hace incidir luz desde una fuente externa que produce el llamado bombeo óptico. Para un determinado valor 
umbral de la luz incidente, la luz emitida es de gran intensidad y coherencia. Este fenómeno es debido al inversión de población, es decir, los átomos en el estado de energía más alto (átomos excitados) decaen al estado de menor energía emitiendo de manera espontánea la luz correspondiente. Para poder mantener esta emisión coherente de radiación es necesario inyectar continuamente energía al sistema.

La obtención de un modelo determinista que describa la fenomenología del Láser se obtiene a partir de las ecuaciones microscópicas que describen la interacción de radiación-materia. Como resultado final se obtiene una ecuación de evolución determinista para el campo eléctrico transmitido que incorpora todos los ingredientes macroscópicos que se observan. El campo eléctrico que es la variable relevante es una cantidad compleja (número complejo). La introducción de las fluctuaciones en la ecuación determinista del sistema Láser se hace siguiendo los pasos habituales en la literatura [38]. Por ejemplo, las fluctuaciones internas (de origen microscópico) presentes en la cavidad son necesarias para entender el proceso de emisión espontánea. Estas fluctuaciones se representan por un término que se suma a la ecuación determinista del campo eléctrico, dicho término también se conoce como ruido aditivo. Existen sin embargo, otros tipos de ruidos llamados externos, éstos como su nombre lo indica, son ajenos al sistema y son controlables en el laboratorio y por tanto sus funciones de correlación pueden presentar efectos de memoria o tiempos finitos de correlación.

El modelo de Láser más simple propuesto en los trabajos antes mencionados, corresponde a una ecuación determinista para el campo eléctrico complejo $E=E_{1}+\mathrm{i} E_{2}$, de un solo modo dado por [2, 3]

$$
\frac{d E}{d t}=-k E+\frac{F}{1+(A / F) I} E
$$

donde $k$ es la razón de decaimiento de la intensidad de radiación en la cavidad del láser, $F$ el factor de ganancia del medio activo, $A$ el parámetro de saturación del medio activo, $I=|\mathbf{E}|^{2}=E_{1}^{2}+E_{2}^{2}$ la intensidad del Láser. La incorporación de las fluctuaciones internas antes mencionadas se realiza a través de una variable estocástica $\xi(t)$ que da cuenta de la emisión espontánea, de modo que la ecuación anterior se vuelve una ecuación 
diferencial estocástica dada por

$$
\frac{d E}{d t}=-k E+\frac{F}{1+(A / F) I} E+\xi(t)
$$

donde $\xi(t)$ es el ruido aditivo que satisface las propiedades de un ruido blanco Gaussiano con valor medio nulo y función de correlación

$$
\left\langle\xi(t) \xi^{*}\left(t^{\prime}\right)\right\rangle=2 \epsilon \delta\left(t-t^{\prime}\right),
$$

siendo $\epsilon$ la intensidad del ruido complejo y $\xi^{*}(t)$ su complejo conjugado. El modelo de láser estudiado por Balle et al. para la detección de señales ópticas débiles es la siguiente [2]

$$
\frac{d E}{d t}=-k E+\frac{F}{1+(A / F) I} E+\mathrm{i} \gamma_{2} E+k_{e} E_{e}+\xi(t)
$$

donde $E_{e}$ es un campo eléctrico externo débil, que se considera como una cantidad real, $k_{e}$ el parámetro de acoplamiento entre el Láser y la señal externa y $\gamma_{2}$ es un parámetro de sintonización entre el campo externo $E_{e}$ y el Láser. Si el factor de ganancia domina al parámetro de saturación, es decir, $A / F \ll 1$, entonces la ec. (3.9) se puede escribir como

$$
\frac{d E}{d t}=\gamma_{1} E-A|\mathbf{E}|^{2} E+\mathrm{i} \gamma_{2} E+k_{e} E_{e}+\xi(t),
$$

donde $\gamma_{1}=F-k>0$, que garantiza un máximo o punto inestable de un "potencial". Para la ec. (3.10) el estado estacionario correspondiente es $I_{s t}=\gamma_{1} / A$.

\subsubsection{Estadística de los tiempos de primer paso}

En este apartado vamos ahora a calcular la estadística de los tiempos de primer paso, asociado al decaimiento del estado inestable del sistema láser (3.10), con la ayuda de la QDT. Como veremos más adelante, la dinámica dada por la Ec. 3.10 es de carácter rotacional aunque dicho efecto no aparece explícitamente en los cálculos teóricos asociados con la estadística de los tiempos de paso reportados en [3]. La descripción teórica reportada en dicha referencia se realiza en el plano complejo de coordenadas 
$\left(E_{1}, E_{2}\right)$. La razón del por qué dichos efectos de rotación no aparecen de manera explícita en el estudio reportado por Balle et al. se puede explicar y entender mejor en un espacio transformado de coordenadas $\left(E_{1}^{\prime}, E_{2}^{\prime}\right)$ del campo eléctrico complejo original $\left(E_{1}, E_{2}\right)$. Esto se mostrará también más adelante.

Por esta razón nuestro estudio en esta sección se hará en términos de una formulación matricial en vez de una formulación en el plano complejo, aunque ambas son equivalentes y conducen a los mismos resultados para la estadística de los tiempos de paso. Comenzamos entonces con la descripción en la aproximación lineal de la dinámica (3.10) la cual se puede escribir en su forma matricial como

$$
\frac{d \mathbf{E}}{d t}=\gamma_{1} \mathbf{E}+W \mathbf{E}+k_{e} \mathbf{E}_{e}+\boldsymbol{\xi}(t)
$$

donde $W$ es una matriz antisimétrica dada por

$$
W=\left(\begin{array}{cc}
0 & -\gamma_{2} \\
\gamma_{2} & 0
\end{array}\right)
$$

y los vectores $\mathbf{E}=\left(E_{1}, E_{2}\right), \mathbf{E}_{e}=\left(E_{e}, 0\right), \boldsymbol{\xi}(t)=\left(\xi_{1}, \xi_{2}\right)$, tal que

$$
\left\langle\xi_{i}(t) \xi_{j}\left(t^{\prime}\right)\right\rangle=\epsilon \delta_{i j} \delta\left(t-t^{\prime}\right), \quad i, j=1,2 .
$$

donde $\epsilon$ es la intensidad del ruido. En la Fig. (3.3), se muestra la evolución dinámica del sistema Láser de acuerdo con la Ec. (3.11), y el círculo representa el valor de referencia $I_{r}=E_{r 1}^{2}+E_{r 2}^{2}$

Si ahora introducimos el siguiente cambio de variable,

$$
\mathbf{E}^{\prime}=e^{-W t} \mathbf{E},
$$

el cual corresponde a una rotación de las coordenadas $\left(E_{1}, E_{2}\right)$ del plano complejo al espacio de coordenadas $\left(E_{1}^{\prime}, E_{2}^{\prime}\right)$, ya que $R(t) \equiv e^{W t}$ es una matriz de rotación (ver Apéndice $\mathrm{B}$ ) de tal forma que $R^{-1}(t) \equiv e^{-W t}$ es su inversa, donde

$$
R(t)=\left(\begin{array}{cc}
\cos \gamma_{2} t & \operatorname{sen} \gamma_{2} t \\
-\operatorname{sen} \gamma_{2} t & \cos \gamma_{2} t
\end{array}\right)
$$

En el espacio transformado de coordenadas la Ec. tipo Langevin (3.11 se transforma en

$$
\frac{d \mathbf{E}^{\prime}}{d t}=\gamma_{1} \mathbf{E}^{\prime}+k_{e} \mathbf{E}_{e}^{\prime}+\boldsymbol{\xi}^{\prime}(t)
$$




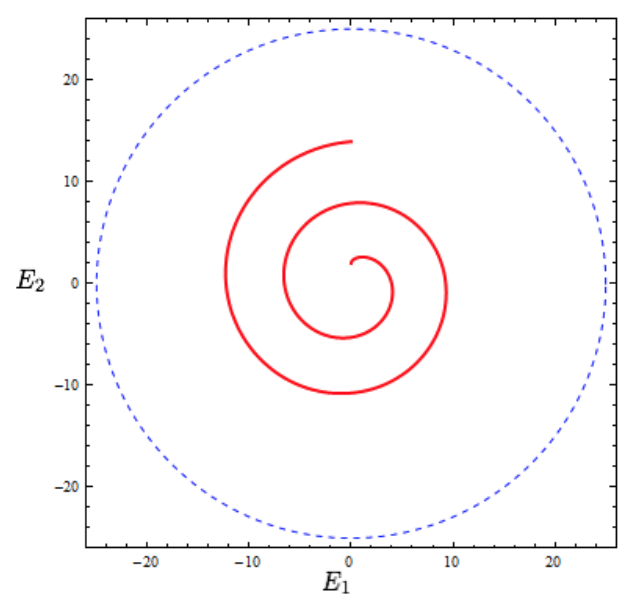

Figura 3.3: Evolución dinámica del sistema Láser (3.11) en el plano complejo $\left(E_{1}, E_{2}\right)$. El círculo de líneas punteadas representa el valor de referencia $I_{r}=E_{1 r}^{2}+E_{2 r}^{2}$

siendo en este caso $\mathbf{E}_{e}^{\prime}=R^{-1}(t) \mathbf{E}_{e}$ y $\boldsymbol{\xi}^{\prime}(t)=R^{-1}(t) \boldsymbol{\xi}(t)$; y como consecuencia tanto el campo eléctrico externo así como el ruido interno sufren los efectos de la rotación. La transformación aplicada elimina la matriz antisimétrica $W$ de la ec. (3.11) y las absorben el campo eléctrico externo y el ruido interno, a través de la matriz de rotación.

Debido a la transformación (3.14), el módulo al cuadrado del campo eléctrico $I=|\mathbf{E}|^{2}$ permanece invariante en el espacio transformado, esto es $I=I^{\prime}$, donde $I^{\prime}=\left|\mathbf{E}^{\prime}\right|^{2}=E_{1}^{\prime 2}+E_{2}^{\prime 2}$. En la Fig. (3.4) se muestran dos dinámicas del sistema Láser (3.16) en el plano complejo $\left(E_{1}^{\prime}, E_{2}^{\prime}\right)$ cuando la intensidad del campo eléctrico externo se compara con la del ruido interno. La figura se obtiene de los cálculos de la simulación numérica de la ec. (3.16), véase la Ref. [39, 40]

Si la magnitud del campo eléctrico externo es menor o igual a la intensidad del ruido interno, entonces la dinámica es prácticamente una línea recta como se muestra en la Fig. (3.4)a, y por lo tanto los efectos de la rotación son prácticamente imperceptibles. En el caso contrario, si la amplitud del campo eléctrico es dominante sobre el ruido interno entonces la trayectoria es rotacional en forma de "bucles", tal como se muestra en la 

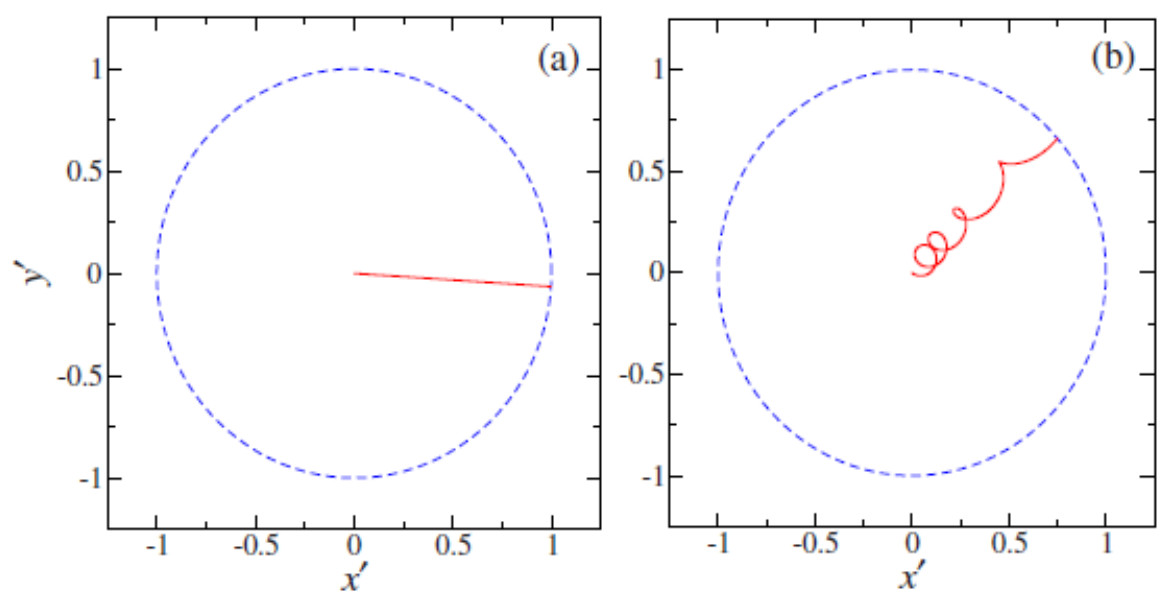

Figura 3.4: Evolución dinámica del sistema láser (3.16 en el espacio trasformado $\left(x^{\prime}=E_{1}^{\prime}, y^{\prime}=E_{2}^{\prime}\right)$. (a) Trayectoria recta para valores de $E_{e}=\epsilon=0.0001$. (b) Trayectoria rotacional obtenida para valores de $E_{e}=1.0$ y $\epsilon=0.001$.

Fig. 3.4 b.

Como podemos observar de las figuras anteriores, solamente cuando la intensidad del campo eléctrico externo es menor o igual que la intensidad del ruido interno $\left(E_{e} \leq \epsilon\right)$, los efectos de rotación en la evolución dinámica del sistema Láser no son apreciables. Por esta razón la estadística de los tiempos de paso en [3] no contiene los efectos de rotación del sistema. De ahora en adelante, vamos a calcular dicha estadística en el espacio transformado del plano complejo y haremos uso de estos resultados para aplicar el criterio de detección propuesto por Balle et al.

Calculemos ahora el tiempo de paso requerido por la intensidad $I(t)$ en alcanzar el valor de referencia, donde $I_{s t}=\gamma_{1} / A$ es el valor del estado estacionario. El $2 \%$ del valor del estado estacionario garantiza estar en la región lineal y lejos del estado estacionario. Este valor es propuesto del experimento en la Ref. [4].

Para tal propósito, partimos de la solución de la Ec. (3.16) dada por

$$
\mathbf{E}^{\prime}(t)=\mathbf{h}^{\prime}(t) e^{\gamma_{1} t}
$$


donde $\mathbf{h}^{\prime}(t)=\left(h_{1}^{\prime}(t), h_{2}^{\prime}(t)\right)$, tal que

$$
\mathbf{h}^{\prime}(t)=\int_{0}^{t} e^{-\gamma_{1} s} R^{-1}(s)\left[k_{e} \mathbf{E}_{e}+\boldsymbol{\xi}(s)\right] d s,
$$

suponiendo condiciones iniciales fijas tal que $\mathbf{E}^{\prime}(0)=0$. En el análisis cualitativo de la QDT, hemos visto que la escala de tiempo característico del decaimiento del estado inestable se lleva a cabo en el límite de tiempos largos, de modo que para valores pequeños del campo externo $\mathbf{E}_{e} \mathrm{y}$ del ruido interno $\boldsymbol{\xi}(t)$, tenemos que

$$
\lim _{t \rightarrow \infty} \frac{d \mathbf{h}^{\prime}}{d t}=\lim _{t \rightarrow \infty} e^{-\gamma_{1} t} R^{-1}(t)\left[k_{e} \mathbf{E}_{e}+\boldsymbol{\xi}(t)\right] \rightarrow 0 .
$$

Este resultado nos indica que en el régimen de tiempos largos $\mathbf{h}^{\prime}(t)$ llega ser una constante, es decir $\mathbf{h}^{\prime}(\infty)=\mathbf{h}^{\prime}$, donde en este caso $\mathbf{h}^{\prime}=\left(h_{1}^{\prime}, h_{2}^{\prime}\right)$ es una variable aleatoria Gaussiana (VAG). Por tanto en dicho régimen de aproximación, el proceso estocástico dado por (3.17) se transforma en un proceso cuasi-determinista dado por $\mathbf{E}^{\prime}(t)=\mathbf{h}^{\prime} e^{\gamma_{1} t}$ de tal manera que $\mathbf{h}^{\prime}$ juega el papel de una condición inicial efectiva. En términos de la intensidad del campo eléctrico $I^{\prime}$ la ecuación cuasi-determinista $\mathbf{E}^{\prime}(t)=\mathbf{h}^{\prime} e^{\gamma_{1} t}$ puede escribirse como

$$
I^{\prime}(t)=h^{\prime 2} e^{2 \gamma_{1} t},
$$

donde $h^{\prime 2} \equiv\left|\mathbf{h}^{\prime}\right|^{2}=h_{1}^{\prime 2}+h_{2}^{\prime 2}$. De la solución 3.20 podemos obtener el tiempo de primer paso requerido por la intensidad en alcanzar el valor de referencia $I_{r}^{\prime}$, es decir, $t=\left(1 / 2 \gamma_{1}\right) \ln \left(I_{r}^{\prime} / h^{\prime 2}\right)$. Este tiempo es también una variable estocástica debido a que es función de $h^{\prime}$. Así que el tiempo medio de primer paso será entonces

$$
\langle t\rangle=\frac{1}{2 \gamma_{1}}\left\langle\ln \left(\frac{I_{r}^{\prime}}{h^{\prime 2}}\right)\right\rangle .
$$

Esta escala de tiempo promedio se puede calcular a través de la densidad de probabilidad marginal $P\left(h^{\prime}\right)$, la cual a su vez requiere de la densidad de probabilidad Gaussiana conjunta

$$
P\left(h_{1}^{\prime}, h_{2}^{\prime}\right)=\frac{1}{2 \pi\left(\operatorname{det} \sigma_{i j}\right)^{2}} \times \exp \left[-\frac{1}{2} \sum_{i, j=1}^{2}\left(\sigma^{-1}\right)_{i j}\left(h_{i}^{\prime}-\left\langle h_{i}^{\prime}\right\rangle\right)\left(h_{j}^{\prime}-\left\langle h_{j}^{\prime}\right\rangle\right)\right],
$$


siendo $\sigma_{i j}=\left\langle h_{i}^{\prime} h_{j}^{\prime}\right\rangle-\left\langle h_{i}^{\prime}\right\rangle\left\langle h_{j}^{\prime}\right\rangle$ la matriz de correlación. De la Ec. 3.18 podemos ver que

$$
\begin{gathered}
\left\langle h_{i}^{\prime}\right\rangle=k_{e} \int_{0}^{\infty} e^{-\gamma_{1} t} R_{i k}^{-1}(t) E_{e k} d t \\
\left\langle h_{i}^{\prime} h_{j}^{\prime}\right\rangle=\left\langle h_{i}^{\prime}\right\rangle\left\langle h_{j}^{\prime}\right\rangle+\int_{0}^{\infty} \int_{0}^{\infty} e^{-\gamma_{1}\left(t+t^{\prime}\right)} R_{i k}^{-1}(t) R_{j l}^{-1}\left(t^{\prime}\right)\left\langle\xi_{k}(t) \xi_{l}\left(t^{\prime}\right)\right\rangle d t d t^{\prime} .
\end{gathered}
$$

De aquí es fácil verificar que

$$
\left\langle h_{1}^{\prime}\right\rangle=\frac{k_{e} E_{e} \gamma_{1}}{\gamma_{1}^{2}+\gamma_{2}^{2}}, \quad\left\langle h_{2}^{\prime}\right\rangle=\frac{k_{e} E_{e} \gamma_{1}}{\gamma_{1}^{2}+\gamma_{2}^{2}},
$$

y

$$
\left\langle h_{i}^{\prime} h_{j}^{\prime}\right\rangle=\left\langle h_{i}^{\prime}\right\rangle\left\langle h_{j}^{\prime}\right\rangle+\frac{\epsilon}{2 \gamma_{1}} \delta_{i j} .
$$

La Ec. 3.26 nos muestra que las variables $h_{i}^{\prime}$ son independientes y por tanto la matriz de correlación $\sigma_{i j}=\left(\epsilon / 2 \gamma_{1}\right) \delta_{i j}$ es una matriz diagonal, cuyos elementos están dados por $\sigma^{2} \equiv \sigma_{i i}=\epsilon / 2 \gamma_{1}$. Bajo estas condiciones la densidad de probabilidad dada $(3.22)$ se reduce a la simple expresión

$$
P\left(h_{1}^{\prime}, h_{2}^{\prime}\right)=\frac{1}{2 \pi \sigma^{2}} e^{-\left(1 / 2 \sigma^{2}\right)\left[\left(h_{1}^{\prime}-\left\langle h_{1}^{\prime}\right\rangle\right)^{2}+\left(h_{2}^{\prime}-\left\langle h_{2}^{\prime}\right\rangle\right)^{2}\right]} .
$$

Para calcular $P\left(h^{\prime}\right)$ hacemos uso de la transformación

$$
P\left(h_{1}^{\prime}, h_{2}^{\prime}\right) d h_{1}^{\prime} d h_{2}^{\prime} \rightarrow P\left(h^{\prime}, \theta\right) J\left(h^{\prime}, \theta\right) d h^{\prime} d \theta,
$$

donde $J\left(h^{\prime}, \theta\right)$ representa la transformación Jacobiana del espacio de coordenadas $\left(h_{1}^{\prime}, h_{2}^{\prime}\right)$ al espacio de coordenadas polares $\left(h^{\prime}, \theta\right)$, siendo $h_{1}^{\prime}=$ $h^{\prime} \cos \theta$ y $h_{2}^{\prime}=h^{\prime} \sin \theta$. Se puede mostrar que el Jacobiano de la transformación es $J\left(h^{\prime}, \theta\right)=h^{\prime}$, y después de efectuar la integración marginal de $P\left(h^{\prime}, \theta\right)$ con respecto al ángulo $\theta$ obtenemos finalmente que

$$
P\left(h^{\prime}\right)=\frac{h^{\prime}}{\sigma^{2}} I_{0}\left(p^{\prime} h^{\prime} / \sigma^{2}\right) e^{-\left(1 / 2 \sigma^{2}\right)\left(h^{\prime 2}+p^{\prime 2}\right)^{2}},
$$

donde $p^{\prime 2}=\left\langle h_{1}^{\prime}\right\rangle^{2}+\left\langle h_{2}^{\prime}\right\rangle^{2}=\left(k_{e} E_{e}\right)^{2} /\left(\gamma_{1}^{2}+\gamma_{2}^{2}\right)$, y la función $I_{0}(x)$ es la función modificada de Bessel de orden cero [41]. 
Para calcular la estadística de los tiempos de paso necesitamos los dos primeros momentos $\langle t\rangle$ y $\left\langle t^{2}\right\rangle$, los cuales se pueden calcular a través de la función generadora de momentos (FGM) que definimos como $G\left(2 \gamma_{1} \nu\right)=$ $\left\langle e^{-2 \gamma_{1} \nu t}\right\rangle$. Sustituyendo la expresión del tiempo de paso obtenido de la ec. (3.20 vemos que $G\left(2 \gamma_{1} \nu\right)=\left\langle\left(I_{r}^{\prime} / h^{\prime 2}\right)^{-\nu}\right\rangle$.

La FGM se calcula explícitamente de la densidad de probabilidad $P\left(h^{\prime}\right)$ dando como resultado (ver Apéndice $\mathrm{A}$ )

$$
\begin{aligned}
G\left(2 \gamma_{1} \nu\right) & =\left(\frac{I_{r}^{\prime}}{2 \sigma^{2}}\right)^{-\nu} e^{-\beta^{2} / 2} \sum_{m=0}^{\infty} \frac{\Gamma(m+\nu+1)}{(m !)^{2}}\left(\frac{\beta^{2}}{2}\right)^{m} \\
& =G_{0}\left(2 \gamma_{1} \nu\right) e^{-\beta^{2} / 2} M\left(\nu+1,1, \beta^{2} / 2\right),
\end{aligned}
$$

donde $G_{0}\left(2 \gamma_{1} \nu\right)=\left(I_{r}^{\prime} / 2 \sigma^{2}\right)^{-\nu} \Gamma(\nu+1)$ es la función generadora de momentos en ausencia del campo eléctrico, $M\left(\nu+1,1, \beta^{2} / 2\right)$ la función hipergeométrica confluente de Kummer y $\beta$ es

$$
\beta^{2}=\frac{p^{2}}{\sigma^{2}}=\frac{2 \gamma_{1}}{\left(\gamma_{1}^{2}+\gamma_{2}^{2}\right)} \frac{\left(k_{e} E_{e}\right)^{2}}{\epsilon},
$$

de donde podemos observar que el parámetro $\beta^{2} \sim\left(k_{e} E_{e}\right)^{2} / \epsilon$. La estadística de los tiempos de paso se obtiene entonces de la FGM mediante las siguientes derivadas

$$
\left\langle 2 \gamma_{1} t\right\rangle=-\left.\frac{d G}{d \nu}\right|_{\nu=0}, \quad\left\langle\left(2 \gamma_{1} t\right)^{2}\right\rangle=\left.\frac{d^{2} G}{d \nu^{2}}\right|_{\nu=0},
$$

obteniéndose después de cierta álgebra, la siguiente expresión

$$
\left\langle 2 \gamma_{1} t\right\rangle=\left\langle 2 \gamma_{1} t\right\rangle_{0}+\sum_{m=1}^{\infty} \frac{(-1)^{m}}{m m !}\left(\frac{\beta^{2}}{2}\right)^{m}
$$

donde

$$
\left\langle 2 \gamma_{1} t\right\rangle_{0}=\ln \left(\frac{\gamma_{1} I_{r}^{\prime}}{\epsilon}\right)-\psi(1),
$$

es el MFPT en ausencia de la fuerza externa y $-\psi(1)=0.577$ es la constante de Euler. Para la varianza del tiempo de primer paso definido por 
$\left\langle\left(2 \gamma_{1} \Delta t\right)^{2}\right\rangle \equiv\left\langle\left(2 \gamma_{1} t\right)^{2}\right\rangle-\left\langle 2 \gamma_{1} t\right\rangle^{2}$, obtenemos lo siguiente

$$
\begin{aligned}
\left\langle\left(2 \gamma_{1} \Delta t\right)^{2}\right\rangle & =\psi^{\prime}(1)+2 \sum_{m=2}^{\infty}\left(\sum_{k=1}^{m-1} \frac{1}{k}\right) \frac{(-1)^{m}}{m m !}\left(\frac{\beta^{2}}{2}\right)^{m} \\
& -\left[\sum_{m=1}^{\infty} \frac{(-1)^{m}}{m m !}\left(\frac{\beta^{2}}{2}\right)^{m}\right]^{2}
\end{aligned}
$$

donde $\psi^{\prime}(1)=\pi^{2} / 6=1.644$. De acuerdo con la QDT, la estadística de los tiempos de paso es válida en el límite de tiempos largos y para valores pequeños de las amplitudes tanto del campo eléctrico como del ruido interno. Podemos observar que las Ecs. (3.33) y 3.35) no contienen términos oscilantes, que describan el carácter rotacional de la dinámica descrita por la Ec. (3.16).

\subsubsection{Detección de señales ópticas débiles}

Balle et al. propusieron un criterio para la detección de señales débiles, el cual está relacionado con la estadística de los tiempo paso y se define como

$$
\left[\langle t\rangle_{\beta_{c}}-\langle t\rangle_{\beta=0}\right]^{2} \geq\left\langle(\Delta t)^{2}\right\rangle_{\beta=0}
$$

este criterio establece que la reducción del tiempo de paso $\langle t\rangle$ debe ser mayor o igual a la máxima varianza. Esta propuesta tiene que ver con la elección de los valores del tiempo de paso y de la varianza en la región de $\beta$ pequeña, donde el ruido es dominante (ver Fig. (3.5)).

Una vez establecido dicho criterio, surge entonces la siguiente pregunta: ¿Qué debemos entender por una señal débil? para responder a esta pregunta debemos recordar el parámetro $\beta^{2} \sim\left(k_{e} E_{e}\right)^{2} / \epsilon$, luego entonces, la amplitud de la señal externa debe ser pequeña comparada con la amplitud o intensidad del ruido interno. Así que para que la amplitud de la señal óptica sea débil, debe de ser menor si acaso igual, a la del ruido interno, es decir $\beta^{2} \leq 1$. Esta condición nos dice que la dinámica del sistema debe ser dominada por el ruido interno, luego entonces, en esta aproximación, tanto la escala de tiempo (3.33) como la varianza 3.35 se pueden escribir como

$$
\left\langle 2 \gamma_{1} t\right\rangle=\left\langle 2 \gamma_{1} t\right\rangle_{0}-\frac{\beta^{2}}{2}
$$




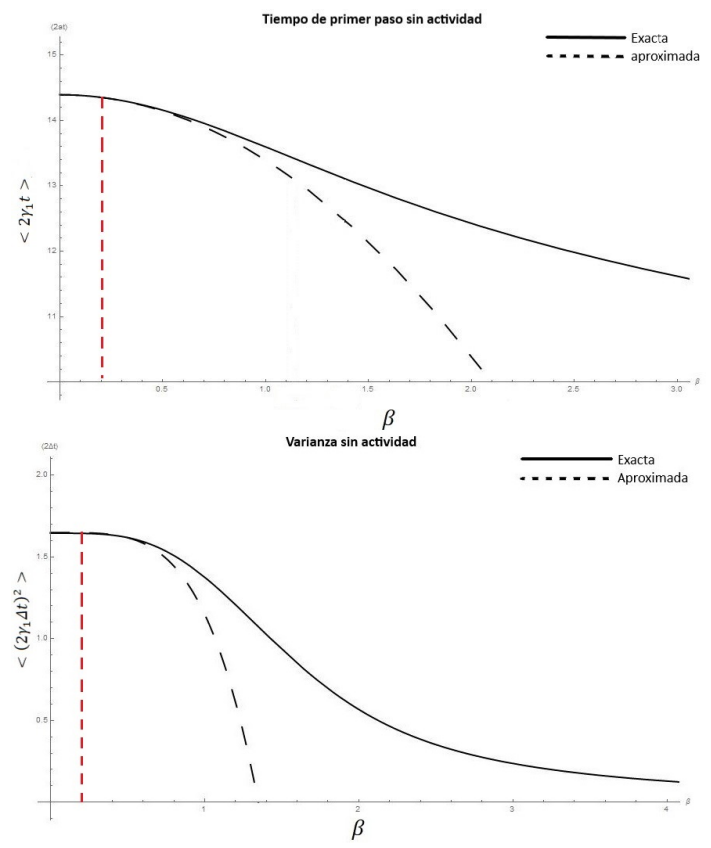

Figura 3.5: Se ilustra la gráfica de las expresiones exactas y aproximadas, tanto para el tiempo de paso, como para la varianza. La línea roja marca la región donde los valores de $\beta$ son pequeños.

$$
\left\langle\left(2 \gamma_{1} \Delta t\right)^{2}\right\rangle=\psi^{\prime}(1)-\frac{\beta^{4}}{8}
$$

Sustituyendo estas expresiones en el criterio 3.36 y quedándonos solo al orden más bajo de $\beta$, así que el valor crítico de $\beta_{c}$ tiene el valor aproximado de

$$
\beta_{c} \geq\left[4 \psi^{\prime}(1)\right]^{1 / 4}=1.6
$$

el cual corresponde al mismo valor reportado en [3]. El criterio (3.36) establece que por abajo de dicho valor crítico no hay detección y para valores mayores que dicho valor, la detección se realiza de manera eficiente. De manera que, la amplitud débil de la señal se debe detectar para cuando $\beta_{c}=1.6$. Es importante comentar que si la amplitud del campo externo es mayor que la intensidad del ruido, $\beta^{2}>1$, entonces la dinámica debe estar 
dominada por la evolución determinista. En este caso la expresión asintótica de la FGM estará dada por $G\left(2 \gamma_{1} \nu\right)=\left(\alpha / \beta^{2}\right)^{-\nu}$ con $\alpha=2 \gamma_{1} I_{r}^{\prime} / \epsilon$, luego entonces el tiempo de paso y su varianza estarán dados por

$$
\begin{aligned}
& \left\langle 2 \gamma_{1} t\right\rangle=\ln \left(\frac{\alpha}{\beta^{2}}\right), \\
& \left\langle\left(2 \gamma_{1} \Delta t\right)^{2}\right\rangle=\frac{4}{\beta^{2}} .
\end{aligned}
$$

En la Fig 3.6, mostramos el valor crítico de $\beta$ correspondiente a la unión entre las dos escalas de tiempo (3.37) y (3.40). Los valores de los parametros son obtenidos de la Ref. [3], donde $F=1.4 \times 10^{7} \mathrm{sec}^{-1}, k=1.2 \times 10^{6} \mathrm{sec}^{-1}$ tal que $\gamma_{1}=1.28 \times 10^{7} \mathrm{sec}^{-1}, I_{r}^{\prime}=0,02 \gamma_{1} / A \operatorname{con} A=2.6 \times 10^{6} \mathrm{sec}^{-1} \mathrm{y}$ $\epsilon=0.004 \mathrm{sec}^{-1}$. Dando como resultado $\alpha=1.794 \times 10^{7}$. 


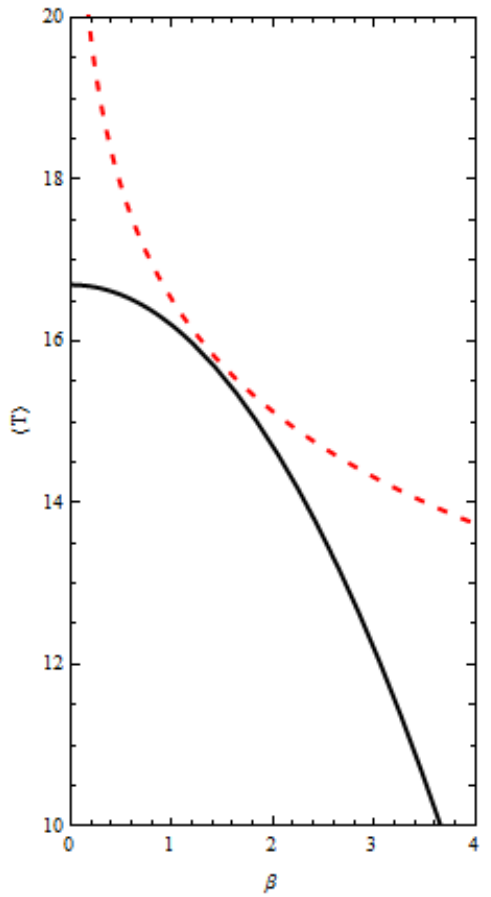

Figura 3.6: Comparación entre el MFPT como función de $\beta$ obtenida de la Ec. (3.37) (línea solida) y el tiempo determinista Ec.(3.40) (línea punteada), para el valor de $\alpha=1.794 \times 10^{7}$. El punto de unión entre ambas es aproximadamente el valor de $\beta_{c}=1.6$. El valor de $\alpha$ es obtenido de $\alpha=2 \gamma_{1} I_{r}^{\prime} / \epsilon$ con los valores $F=1.4 \times 10^{7} \mathrm{sec}^{-1}, k=1.2 \times 10^{6} \mathrm{sec}^{-1}$ tal que $\gamma_{1}=1.28 \times 10^{7} \mathrm{sec}^{-1}, I_{r}^{\prime}=0,02 \gamma_{1} / A \operatorname{con} A=2.6 \times 10^{6} \sec ^{-1} \mathrm{y}$ $\epsilon=0.004 \mathrm{sec}^{-1}$, obtenidos de la Ref. [3]. 


\section{Capítulo 4}

\section{Partículas activas en la detección de señales débiles}

\subsection{Introducción}

En el capítulo anterior hemos estudiado el problema de la detección de señales ópticas débiles en el proceso de encendido de un sistema láser. Para tal propósito, hemos utilizado un criterio relacionado con la estadística de los tiempos de primer paso que caracteriza dicho proceso de encendido. Debemos tomar en cuenta que el proceso de encendido es equivalente al proceso de decaimiento de un estado inestable que experimenta la intensidad de la luz Láser por efecto de las fluctuaciones estocásticas representadas por la emisión espontánea. Tomando en cuenta las ideas físicas expuestas en el proceso de decaimiento del sistema Láser como un mecanismo para detectar señales ópticas débiles, nos preguntamos ahora, si el proceso de decaimiento o relajación de un estado inestable de partículas activas con carga eléctrica, por efecto de pequeñas fluctuaciones y por la misma actividad de dichas partículas, podría ser un mecanismo alternativo capaz de detectar amplitudes débiles de un campo eléctrico externo. Es evidente que esta idea intuitiva no es fortuita, sino surge porque se trata del proceso de decaimiento de un estado inestable; y de ahí la pregunta, si dicho proceso podría ser capaz de detectar amplitudes débiles de un campo eléctrico externo, similar al caso del sistema Láser. En esta tesis partimos de la 
hipótesis que dicho proceso de detección sea posible. Evidentemente la respuesta contundente lo tiene el experimento.

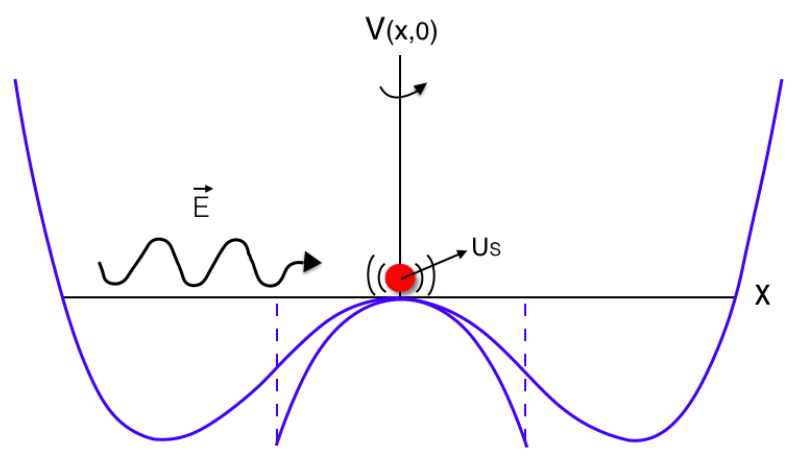

Figura 4.1: Decaimiento del estado inestable de una partícula activa en presencia de un campo eléctrico constante, en un potencial biestable $V(x, 0)$. El potencial $V(x, y)$ se genera mediante la rotación alrededor del eje $z$, siendo el eje $y$ perpendicular al plano.

De manera muy similar al sistema Láser estudiado en el capítulo anterior, usamos el mismo criterio propuesto por Balle et al. 3] en términos de la estadística de los tiempos de paso para caracterizar la dinámica transitoria del decaimiento del estado inestable de las partículas activas con carga eléctrica inmersas en un baño térmico. Siguiendo las ideas físicas antes mencionadas del sistema láser, el mecanismo de detección consiste esencialmente en lo siguiente: una vez desencadenado el proceso de decaimiento por efecto del ruido interno, éste pueda ser capaz de detectar la señal débil (campo eléctrico) incidente. Otra pregunta que respondemos en este trabajo es la siguiente: ¿qué resultados nos arroja el criterio de detección acerca de la actividad de las partículas, comparadas con la pasividad de las mismas?

En Nuestro problema consideremos un enjambre de partículas brownianas activas e independientes con carga eléctrica localizadas al tiempo $t=0$ en el estado instable de un potencial biestable de dos dimensiones (2D) dada por $V(x, y)=-\frac{a_{0}}{2}\left(x^{2}+y^{2}\right)+\frac{b_{0}}{4}\left(x^{2}+y^{2}\right)^{2}$, donde $a_{0}, b_{0}>0$, 
y $r^{2}=|r|^{2}=x^{2}+y^{2}$ es el módulo al cuadrado del vector de posición $\mathbf{r}=(x, y)$. En la Fig. 4.1 se muestra la gráfica de dicho potencial en una dimensión. Debemos enfatizar que para el criterio teórico de detección antes mencionado, es suficiente con caracterizar el decaimiento del estado inestable en la aproximación lineal del potencial, de tal manera que la barrera absorbente del potencial será un círculo de radio $R^{2}=k_{0} r_{s t}^{2}$, donde $r_{s t}^{2}$ es el valor del estado estacionario y $k_{0}$ una constante. En nuestra propuesta supondremos que el movimiento activo de la partícula browniana se lleva acabo únicamente mediante un grado de libertad adicional a través del ángulo azimutal $\varphi(t)$, que a su vez es también una variable estocástica. Luego entonces el movimiento activo de la partícula introduce un grado de libertad adicional $\varphi(t)$ produciendo un movimiento estocástico de rotación alrededor del eje $z$, como consecuencia de las torcas térmicas internas. Por esta razón se introducen dos ecuaciones de Langevin con ruido aditivo que describirán la dinámica estocástica de la partícula activa, una para el movimiento browniano de traslación y la otra para el movimiento browniano activo de rotación.

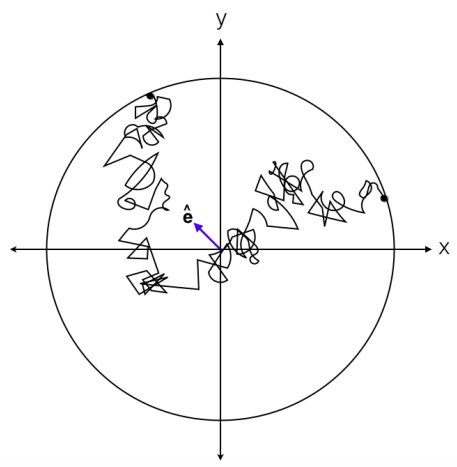

Figura 4.2: Representación esquemática en el plano $(x, y)$ del decaimiento del estado inestable de una partícula activa. La circunferencia representa la barrera del potencial lineal en dos dimensiones. El vector unitario ê $(t)$ es un vector sobre el plano que rota de manera aleatoria.

Consideremos entonces una partícula browniana esférica activa de masa $m$, radio $\rho$ y carga eléctrica $q_{0}$, inmersa en un baño térmico de temperatura $T$ y localizada inicialmente en el estado inestable del potencial biestable de 
$2 \mathrm{D}$ definido anteriormente. Al tiempo inicial $t=0$, comienza el proceso de decaimiento del estado inestable por efecto de las fluctuaciones estocásticas de traslación y rotación. Inmediatamente después de abandonar el estado inestable la partícula es sometida a la acción de una señal eléctrica externa $\mathbf{E}_{0}$ (vector en el plano $x y$ ), cuya amplitud debe ser menor o del mismo orden que el ruido interno total. Por simplicidad supondremos que $\mathbf{E}_{0}=$ $\frac{1}{\sqrt{2}}\left(E_{0}, E_{0}\right)$, de modo que $\left|\mathbf{E}_{0}\right|=E_{0}$, y la amplitud de la fuerza eléctrica $\mathbf{F}_{0}=q_{0} \mathbf{E}_{0}$ será $\left|\mathbf{F}_{0}\right|=F_{0}=q_{0} E_{0}$.

Vale la pena comentar que el sistema físico que se propone como un mecanismo alternativo para detectar señales eléctricas débiles es diferente al sistema láser estudiado en el capítulo anterior. Sin embargo, la idea del proceso de detección es muy similar en ambos sistemas, puesto que dicho proceso de detección tiene que ver con la relajación dinámica de un estado inestable, por efecto de fluctuaciones estocásticas. Luego entonces, usaremos el mismo criterio de detección propuesto para el sistema láser y la QDT para la caracterización dinámica del decaimiento del estado inestable de la partícula activa. La dinámica de Langevin en 2D asociada a la partícula activa en este caso se puede escribir como

$$
m \dot{\mathbf{v}}=-\gamma_{v} \mathbf{v}+a_{0} \mathbf{r}-b_{0} r^{2} \mathbf{r}+\mathbf{F}_{0}+\gamma_{v} U_{s} \hat{\mathbf{e}}(t)+\tilde{\mathbf{f}}(t)
$$

donde $\gamma_{v}=6 \pi \eta \rho$ es el coeficiente de fricción ( $\eta$ la viscosidad del fluido), $\hat{\mathbf{e}}(t)=\left(e_{1}(t), e_{2}(t)\right)$ el vector unitario instantáneo en la dirección de nado, con origen en el centro de la partícula activa, $U_{s}$ la magnitud de la velocidad de nado que suponemos constante. El ruido térmico traslacional $\tilde{\mathbf{f}}(t)$ suponemos que satisface las propiedades de ruido blanco gaussiano con valor medio cero y función de correlación

$$
\begin{aligned}
\langle\tilde{\mathbf{f}}(t)\rangle & =0 \\
\left\langle\tilde{f}_{i}(t) \tilde{f}_{j}(t)\right\rangle & =2 \gamma_{v} k_{B} T \delta_{i j} \delta\left(t-t^{\prime}\right),
\end{aligned}
$$

siendo $k_{B}$ la constante de Boltzmann.

Por otro lado, de la mecánica clásica se sabe que la dinámica del vector unitario $\hat{\mathbf{e}}(t)$ satisface [42, 43, 44]

$$
\dot{\hat{\mathbf{e}}}(\mathbf{t})=\mathbf{\Omega} \times \hat{\mathbf{e}}(\mathbf{t}),
$$


donde $\boldsymbol{\Omega}$ es la velocidad angular de la partícula browniana activa. La ecuación de Langevin para la velocidad angular se propone como

$$
I \dot{\boldsymbol{\Omega}}=-\gamma_{\Omega} \boldsymbol{\Omega}+\gamma_{\Omega} \boldsymbol{\Omega}_{e}+\tilde{\mathbf{g}}(t),
$$

siendo ahora $I$ el momento de inercia, $\boldsymbol{\Omega}_{e}$ la velocidad angular debido a la fuerza externa, y $\tilde{\mathbf{g}}(t)$ representa las torcas térmicas (ruido interno rotacional) que también satisfacen las propiedades de ruido blanco gaussiano, tal que

$$
\begin{aligned}
\langle\tilde{\mathbf{g}}(t)\rangle & =0 \\
\left\langle\tilde{g}_{i}(t) \tilde{g}_{j}(t)\right\rangle & =2 \gamma_{\Omega} k_{B} T \delta_{i j} \delta\left(t-t^{\prime}\right),
\end{aligned}
$$

donde $\gamma_{\Omega}=8 \pi \eta \rho^{3}$ es el coeficiente de fricción rotacional. De la hidrodinámica clásica $\boldsymbol{\Omega}_{e}=\frac{1}{2} \nabla \times \mathbf{F}_{0}=0$ ya que $\mathbf{F}_{0}=$ cte [45]. Usualmente el movimiento browniano de las partículas activas se describe en un ambiente con un valor pequeño del número de Reynolds (o coeficiente de fricción grande), y por tanto en el régimen sobreamortiguado las ecuaciones de Langevin anteriores se reducen a

$$
\begin{aligned}
\dot{\mathbf{r}} & =a \mathbf{r}-b r^{2} \mathbf{r}+\mathbf{F}+U_{s} \hat{\mathbf{e}}(t)+\mathbf{f}(t), \\
\boldsymbol{\Omega} & =\mathbf{g}(t),
\end{aligned}
$$

en este caso $a=\frac{a_{0}}{\gamma_{v}}, b=\frac{b_{0}}{\gamma_{v}}, \mathbf{F}=\frac{\mathbf{F}_{0}}{\gamma_{v}}$ y $\mathbf{f}(t)=\frac{\tilde{\mathbf{f}}}{\gamma_{v}}, \mathbf{g}(t)=\frac{\tilde{\mathbf{g}}(t)}{\gamma_{\Omega}}$, de modo que

$$
\left\langle f_{i}(t) f_{j}(t)\right\rangle=2 D_{B} \delta_{i j} \delta\left(t-t^{\prime}\right),
$$

similarmente

$$
\left\langle g_{i}(t) g_{j}(t)\right\rangle=2 D_{\Omega} \delta_{i j} \delta\left(t-t^{\prime}\right),
$$

donde ahora $D_{B}=\frac{k_{B} T}{\gamma_{v}}$ el coeficiente de difusión de Einstein y $D_{\Omega}=\frac{k_{B} T}{\gamma_{\Omega}}$ el coeficiente de difusión rotacional. Es importante mencionar que, de acuerdo con las ecs. 4.2 y 4.5), la intensidad del ruido interno traslacional está dado por $\lambda_{t}=\gamma_{v} k_{B} T$ y la intensidad del ruido rotacional por $\lambda_{r}=\gamma_{\Omega} k_{B} T$, y por tanto $D_{B}=\frac{\lambda_{t}}{\gamma_{v}^{2}}$ y $D_{\Omega}=\frac{\lambda_{r}}{\gamma_{\Omega}^{2}}$.

Debemos notar que las partículas se mueven en el plano $x y$ y por tanto la torca aleatoria apunta a lo largo del eje $z$, luego entonces $\mathbf{g}=g(t) \mathbf{k}$, con 
lo que la ec. 4.7) será $\boldsymbol{\Omega}=g(t) \mathbf{k}$. Sustituyendo esta expresión en la ec. 4.3) y usando el hecho de que $\hat{\mathbf{e}}(t)=(\cos \varphi, \sin \varphi)$, podemos mostrar que dicha ecuación se transforma en una simple ecuación diferencial estocástica para el ángulo azimutal dada por $\dot{\varphi}(t)=g(t)$. Esta ecuación representa un proceso de Wiener cuya densidad de probabilidad conjunta está dada por $G\left(\varphi, t ; \varphi^{\prime}, t^{\prime}\right)=P\left(\varphi, t \mid \varphi^{\prime}, t^{\prime}\right) P\left(\varphi^{\prime}, t^{\prime}\right)$, donde $P\left(\varphi, t \mid \varphi^{\prime}, t^{\prime}\right)$ es la densidad de probabilidad condicional (DPC) de encontrar a la variable $\varphi$ en el intervalo $(\varphi, \varphi+d \varphi)$, al tiempo $t$, dado que al tiempo $t^{\prime}$ tiene el valor $\varphi^{\prime}$; siendo $P\left(\varphi^{\prime}, t^{\prime}\right)$ la densidad de probabildad de $\varphi^{\prime}$ al tiempo $t^{\prime}$. Ambas están dadas por

$$
\begin{gathered}
P\left(\varphi, t \mid \varphi^{\prime}, t^{\prime}\right)=\frac{1}{\sqrt{4 \pi D_{\Omega}\left(t-t^{\prime}\right)}} \exp \left(\frac{-\left(\varphi-\varphi^{\prime}\right)^{2}}{4 D_{\Omega}\left(t-t^{\prime}\right)}\right), \quad t^{\prime}<t \\
P\left(\varphi^{\prime}, t^{\prime}\right)=\frac{1}{\sqrt{4 \pi D_{\Omega} t^{\prime}}} \exp \left(\frac{-\left(\varphi^{\prime}-\varphi_{0}\right)^{2}}{4 D_{\Omega} t^{\prime}}\right) .
\end{gathered}
$$

Aquí $\varphi^{\prime}(0) \equiv \varphi_{0}$ es el valor inicial al tiempo $t=0$ de $\varphi^{\prime}$, lo que significa $P\left(\varphi^{\prime}, 0\right)=\delta\left(\varphi^{\prime}-\varphi_{0}\right)$

Más adelante requeriremos de las funciones de correlación orientacional $\left\langle e_{k}(t) e_{i}\left(t^{\prime}\right)\right\rangle$ del nadador, las cuales se pueden evaluar mediante la doble integral

$$
\left\langle e_{k}(t) e_{l}\left(t^{\prime}\right)\right\rangle=\int d \varphi \int d \varphi^{\prime} e_{k}(t) e_{l}\left(t^{\prime}\right) G\left(\varphi, t ; \varphi^{\prime}, t^{\prime}\right) .
$$

Usando las densidades de probabilidad (4.10) y (4.11) y el ordenamiento en el tiempo ya sea que $t>t^{\prime}$ o $t<t^{\prime}$, se puede demostrar que para $t>t^{\prime}$ se tiene que

$$
\begin{aligned}
\left\langle e_{1}(t) e_{1}\left(t^{\prime}\right)\right\rangle & =\frac{1}{2} e^{-D_{\Omega}\left(t-t^{\prime}\right)}\left[1+\cos \left(2 \varphi_{0}\right) e^{-4 D_{\Omega} t^{\prime}}\right] \\
\left\langle e_{2}(t) e_{2}\left(t^{\prime}\right)\right\rangle & =\frac{1}{2} e^{-D_{\Omega}\left(t-t^{\prime}\right)}\left[1-\cos \left(2 \varphi_{0}\right) e^{-4 D_{\Omega} t^{\prime}}\right] \\
\left\langle e_{1}(t) e_{2}\left(t^{\prime}\right)\right\rangle & =\frac{1}{2} e^{-D_{\Omega}\left(t-t^{\prime}\right)}\left[\sin \left(2 \varphi_{0}\right) e^{-4 D_{\Omega} t^{\prime}}\right] \\
\left\langle e_{2}(t) e_{1}\left(t^{\prime}\right)\right\rangle & =\frac{1}{2} e^{-D_{\Omega}\left(t-t^{\prime}\right)}\left[\sin \left(2 \varphi_{0}\right) e^{-4 D_{\Omega} t^{\prime}}\right] .
\end{aligned}
$$

Para $t<t^{\prime}$ las funciones de correlación son las mismas pero intercambiando $t$ por $t^{\prime}$ y $t^{\prime}$ por $t$, (ver Apéndice C). 


\subsection{La QDT y el tiempo promedio de primer pa- so}

Tal como hemos mostrado en el capítulo 3, la QDT nos permite caracterizar el proceso de decaimiento del estado inestable en la aproximación lineal del potencial biestable. En este régimen de aproximación, la dinámica de la partícula activa está restringida en los intervalos $-R \leq x \leq R$, $-R \leq y \leq R$, siendo $\mathrm{R}$ la barrera absorbente del potencial, tal que $0 \leq x^{2}+y^{2} \leq R^{2}$, y $R^{2}=k_{0} r_{s t}^{2}$, donde $r_{s t}^{2}$ es el valor del estado estacionario del sistema y la constante $0<k_{0} \ll 1$. Esto nos garantiza que podemos estar tan lejos de la región no lineal o de saturación del potencial biestable. En la aproximación lineal (véase la Fig. 3.2 del capítulo anterior en el caso de 1D) las ecuaciones de Langevin 4.6 y 4.7 se reducen entonces a

$$
\begin{aligned}
\dot{\mathbf{r}} & =a \mathbf{r}+\mathbf{F}+U_{s} \hat{\mathbf{e}}(t)+\mathbf{f}(t), \\
\dot{\varphi} & =g(t) .
\end{aligned}
$$

La solución de la ec. (4.14) se puede escribir como

$$
\mathbf{r}(t)=\mathbf{h}(t) e^{a t},
$$

donde

$$
\mathbf{h}(t)=\mathbf{r}_{0}+\int_{0}^{t} e^{-a t^{\prime}}\left[\mathbf{F}+U_{s} \hat{\mathbf{e}}\left(t^{\prime}\right)+\mathbf{f}\left(t^{\prime}\right)\right] d t^{\prime},
$$

con $\mathbf{r}(0) \equiv \mathbf{r}_{0}$ la posición inicial de la partícula al tiempo $t=0$. De la ec. 4.14 se puede verificar que en ausencia de la fuerza eléctrica externa y del ruido interno traslacional, el término activo introduce un "potencial efectivo" cuyo centro está fuera del origen de coordenadas, siendo las coordenadas de dicho centro el vector $\left(U_{s} / a\right)(\cos \varphi, \sin \varphi)$. Podemos entonces suponer que al tiempo $t=0$ la partícula activa adquiere por sí misma un impulso para estar en la posición $\mathbf{r}_{0}=\left(U_{s} / a\right)\left(\cos \varphi_{0}, \sin \varphi_{0}\right)$.

De acuerdo con la QDT, en el límite de tiempos largos podemos ver que para amplitudes pequeñas de la señal externa, la velocidad de nado y la intensidad del ruido, entonces

$$
\lim _{t \rightarrow \infty} \frac{d \mathbf{h}(t)}{d t}=\lim _{t \rightarrow \infty} e^{-a t}\left[\mathbf{F}+U_{s} \hat{\mathbf{e}}(t)+\mathbf{f}(t)\right] \rightarrow 0 .
$$


Esto significa que en el límite de tiempos largos el proceso estocástico $\mathbf{h}(t)$ se convierte en una variable aleatoria gaussiana constante, debido a que la derivada de una constante es cero. En este caso se puede establecer que en dicho límite de tiempos largos $\mathbf{h}(t)$ llega a ser una constante, es decir, $\mathbf{h}(\infty)=\mathbf{h}=$ cte. Luego entonces el proceso estocástico 4.16) se convierte en un proceso cuasi-determinista dado por $\mathbf{r}(t)=\mathbf{h} e^{a t}$, lo que significa que $\mathbf{h}=\left(h_{1}, h_{2}\right)$ juega el papel de una condición inicial efectiva, véase la ec. 4.17). Esta expresión también se puede escribir en términos del módulo al cuadrado del vector $\mathbf{r}$, es decir,

$$
r^{2}(t)=h^{2} e^{2 a t}
$$

donde $h^{2}=|\mathbf{h}|^{2}=h_{1}^{2}+h_{2}^{2}$. A partir de 4.19$)$, podemos calcular el tiempo de paso que requiere la partícula activa para alcanzar el valor de referencia o barrera absorbente $R$ del potencial, esto es $t=(1 / 2 a) \ln \left(R^{2} / h^{2}\right)$. Como podemos observar este tiempo es función de la variable aleatoria $h$ y por tanto es también aleatorio, así que su valor promedio representa el tiempo promedio de primer paso requerido en alcanzar la barrera $R$ del potencial, el cual está dado por

$$
\langle t\rangle=\frac{1}{2 a}\left\langle\ln \left(\frac{R^{2}}{h^{2}}\right)\right\rangle .
$$

Las propiedades estadísticas del tiempo de paso se pueden calcular a través de la función generadora de momentos definida por $G(2 a \nu)=\left\langle e^{-2 a \nu t}\right\rangle=$ $\left\langle\left(R^{2} / h^{2}\right)^{-\nu}\right\rangle$, que a su vez se puede obtener de la densidad de probabilidad marginal $P(h)$. La densidad de probabilidad marginal se obtiene de la densidad de probabilidad conjunta gaussiana dada por

$$
P\left(h_{1}, h_{2}\right)=\frac{1}{2 \pi \sqrt{\operatorname{det} \sigma_{i j}}} \exp \left[-\frac{1}{2} \sum_{i, j=1}^{2}\left(\sigma^{-1}\right)_{i j}\left(h_{i}-\left\langle h_{i}\right\rangle\right)\left(h_{j}-\left\langle h_{j}\right\rangle\right)\right],
$$

donde $\sigma_{i j}=\left\langle h_{i} h_{j}\right\rangle-\left\langle h_{i}\right\rangle\left\langle h_{j}\right\rangle$ es la matriz de covarianza $(i \neq j)$ y varianza $(i=j)$. Los valores promedio se obtienen de la ec. (4.17), esto es

$$
\begin{aligned}
& \left\langle h_{1}\right\rangle=\frac{U_{s}}{a} \cos \varphi_{0}+\int_{0}^{\infty} e^{-a t}\left[F_{1}+U_{s}\left\langle e_{1}(t)\right\rangle\right] d t, \\
& \left\langle h_{2}\right\rangle=\frac{U_{s}}{a} \sin \varphi_{0}+\int_{0}^{\infty} e^{-a t}\left[F_{2}+U_{s}\left\langle e_{2}(t)\right\rangle\right] d t,
\end{aligned}
$$


donde $\mathbf{F}=\left(F_{1}, F_{2}\right)=\mathbf{F}_{0} / \gamma_{v}$, siendo $F_{1}=F_{2}=\frac{q_{0} E_{0}}{\gamma_{v} \sqrt{2}}$. Así que $|\mathbf{F}|=F=$ $q_{0} E_{0} / \gamma_{v}$, entonces $F_{1}=F_{2}=\frac{F}{\sqrt{2}}$.

$$
\begin{aligned}
& \left\langle e_{1}(t)\right\rangle=\int_{-\infty}^{\infty} \cos \varphi P(\varphi, t) d \varphi=e^{-D_{\Omega} t} \cos \varphi_{0} \\
& \left\langle e_{2}(t)\right\rangle=\int_{-\infty}^{\infty} \sin \varphi P(\varphi, t) d \varphi=e^{-D_{\Omega} t} \sin \varphi_{0} .
\end{aligned}
$$

Por lo tanto

$$
\begin{aligned}
& \left\langle h_{1}\right\rangle=\frac{U_{s}}{a} \cos \varphi_{0}+\frac{F}{\sqrt{2} a}+\frac{U_{s} \cos \varphi_{0}}{\left(a+D_{\Omega}\right)}, \\
& \left\langle h_{2}\right\rangle=\frac{U_{s}}{a} \sin \varphi_{0}+\frac{F}{\sqrt{2} a}+\frac{U_{s} \sin \varphi_{0}}{\left(a+D_{\Omega}\right)} .
\end{aligned}
$$

Por otro lado, la matriz $\sigma_{i j}$ será

$$
\begin{aligned}
\sigma_{i j}=\int_{0}^{\infty} \int_{0}^{\infty} e^{-a\left(t+t^{\prime}\right)} & \left\langle f_{i}(t) f_{j}\left(t^{\prime}\right)\right\rangle d t d t^{\prime} \\
& +U_{s}^{2} \int_{0}^{\infty} \int_{0}^{\infty} e^{-a\left(t+t^{\prime}\right)}\left\langle e_{i}(t) e_{j}\left(t^{\prime}\right)\right\rangle d t d t^{\prime} \\
& \quad-U_{s}^{2} \int_{0}^{\infty} e^{-a t}\left\langle e_{i}(t)\right\rangle d t \int_{0}^{\infty} e^{-a t^{\prime}}\left\langle e_{j}\left(t^{\prime}\right)\right\rangle d t^{\prime} .
\end{aligned}
$$

Teniendo en cuenta los resultados explícitos dados anteriormente, se puede mostrar después de la integración correspondiente que

$$
\begin{gathered}
\sigma_{11}=\frac{D_{B}}{a}+\frac{U_{s}^{2} D_{\Omega}}{2 a\left(a+D_{\Omega}\right)^{2}}-\frac{U_{s}^{2} D_{\Omega} \cos \left(2 \varphi_{0}\right)}{2\left(a+D_{\Omega}\right)^{2}\left(a+2 D_{\Omega}\right)} \\
\sigma_{22}=\frac{D_{B}}{a}+\frac{U_{s}^{2} D_{\Omega}}{2 a\left(a+D_{\Omega}\right)^{2}}-\frac{U_{s}^{2} D_{\Omega} \cos \left(2 \varphi_{0}\right)}{2\left(a+D_{\Omega}\right)^{2}\left(a+2 D_{\Omega}\right)} \\
\sigma_{12}=\sigma_{21}=-\frac{U_{s}^{2} D_{\Omega} \sin \left(2 \varphi_{0}\right)}{2\left(a+D_{\Omega}\right)^{2}\left(a+2 D_{\Omega}\right)} .
\end{gathered}
$$

La condición inicial $\varphi_{0}$ puede tomar todos los valores en el intervalo $[0,2 \pi]$ de modo que, al integrarse en este intervalo para cada una de las funciones $\sin \varphi, \cos \varphi, \sin 2 \varphi, \cos 2 \varphi$ concluimos que

$$
\left\langle h_{1}\right\rangle=\left\langle h_{2}\right\rangle=\frac{F}{\sqrt{2} a},
$$




$$
\begin{gathered}
\sigma_{11}=\sigma_{22}=\frac{D_{B}}{a}+\frac{U_{s}^{2} D_{\Omega}}{2 a\left(a+D_{\Omega}\right)^{2}}, \\
\sigma_{12}=\sigma_{21}=0 .
\end{gathered}
$$

Luego entonces la matriz $\sigma_{i j}$ es diagonal dada por $\sigma_{i j}=\sigma^{2} \delta_{i j}$, donde $\sigma^{2}=\frac{D_{e}}{a} \mathrm{y}$

$$
D_{e}=D_{B}+\frac{U_{s}^{2} D_{\Omega}}{2\left(a+D_{\Omega}\right)^{2}}=D_{B}\left[1+\frac{U_{s}^{2}}{2\left(a+D_{\Omega}\right)^{2}} \frac{D_{\Omega}}{D_{B}}\right],
$$

de tal manera que $D_{e}$ se interpreta como un coeficiente de difusión efectivo o coeficiente de difusión de Einstein rescalado por el factor $K_{a}=[1+$ $\left.U_{s}^{2} D_{\Omega} / 2\left(a+D_{\Omega}\right)^{2} D_{B}\right]$, es decir, $D_{e}=K_{a} D_{B}$. Claramente $D_{e}>D_{B}$ puesto que $K_{a}>1$, lo cual significa que la difusión es mayor en presencia de la actividad de las partículas brownianas. En ausencia de actividad $\left(U_{s}=0\right.$, $\left.D_{\Omega}=0\right)$, el parámetro $K_{a}=1$ y $D_{e}=D_{B}$. Bajo estas condiciones la densidad de probabilidad conjunta se reduce a

$$
P\left(h_{1}, h_{2}\right)=\frac{1}{2 \pi \sigma^{2}} e^{-\left(1 / 2 \sigma^{2}\right)\left[\left(h_{1}+\left\langle h_{1}\right\rangle\right)^{2}+\left(h_{2}+\left\langle h_{2}\right\rangle\right)^{2}\right]} .
$$

La densidad de probabilidad marginal $P(h)$ se puede calcular usando la integración apropiada, dando como resultado

$$
P(h)=\frac{h}{\sigma^{2}} I_{0}\left(\frac{p h}{\sigma^{2}}\right) e^{-\left(h^{2}+p^{2}\right) / 2 \sigma^{2}},
$$

donde $p^{2}=\left\langle h_{1}\right\rangle^{2}+\left\langle h_{2}\right\rangle^{2}=\frac{F^{2}}{a^{2}}$ y $I_{0}(x)$ es la función modificada de Bessel de orden cero [41]. Dada la ec. 4.34) podemos ahora calcular la función generadora de momentos, la cual después de la integración correspondiente obtenemos que

$$
\begin{aligned}
G(2 a \nu) & =\left(\frac{R^{2}}{2 \sigma^{2}}\right)^{-\nu} e^{-\beta_{a}^{2}} \sum_{m=0}^{\infty} \frac{\Gamma(m+\nu+1)}{(m !)^{2}} \\
& =G_{0}(2 a \nu) e^{-\beta_{a}^{2}} M\left(\nu+1,1, \beta_{a}^{2}\right),
\end{aligned}
$$

donde $G_{0}(2 a \nu)=\left(\frac{R^{2}}{2 \sigma^{2}}\right)^{-\nu} \Gamma(\nu+1)$ es la función generadora de momentos en ausencia de la señal externa, $M\left(\nu+1,1, \beta_{a}^{2}\right)$ la función hipergeométrica 
confluente de Kummer [41], y el parámetro

$$
\beta_{a}^{2}=\frac{p^{2}}{2 \sigma^{2}}=\frac{F^{2}}{2 a D_{e}}=\frac{\beta_{0}^{2}}{K_{a}}
$$

donde $\beta_{0}^{2}=\frac{F^{2}}{2 a D_{B}}$ es el parámetro en ausencia de la actividad. La estadística de los tiempos de primer paso, descritos por el valor medio $\langle 2 a t\rangle$ y la varianza definida como $(2 a \Delta t)^{2}=\left\langle(2 a t)^{2}\right\rangle-\langle 2 a t\rangle^{2}$, se pueden obtener de la función generadora de momentos 3.31), es decir

$$
\langle 2 a t\rangle=-\left.\frac{d G(2 a \nu)}{d \nu}\right|_{\nu=0}, \quad\left\langle(2 a t)^{2}\right\rangle=-\left.\frac{d^{2} G(2 a \nu)}{d \nu^{2}}\right|_{\nu=0} .
$$

Después de cierta álgebra, es posible mostrar que el tiempo promedio de primer paso está dado por

$$
\langle 2 a t\rangle=\langle 2 a t\rangle_{0}-e^{-\beta^{2}} \sum_{n=1}^{\infty}[\psi(n+1)-\psi(1)] \frac{\left(\beta_{a}^{2}\right)^{n}}{n !},
$$

donde

$$
\langle 2 a t\rangle_{0}=\ln \left(\frac{a R^{2}}{2 D_{e}}\right)+\gamma
$$

es el tiempo promedio de primer paso en ausencia de la señal externa, $\gamma=-\psi(1)=0.577$ la constante de Euler y $\psi(x)$ es la función psi de Euler [41]. También es posible mostrar que la varianza se puede escribir como

$$
\begin{aligned}
\left\langle(2 a \Delta t)^{2}\right\rangle & =\psi^{\prime}(1)+e^{-\beta_{a}^{2}} \sum_{n=1}^{\infty}[\psi(n+1)-\psi(1)]^{2} \frac{\left(\beta_{a}^{2}\right)^{n}}{n !} \\
& -e^{-\beta_{a}^{2}} \sum_{n=1}^{\infty}\left[\psi^{\prime}(n+1)-\psi^{\prime}(1)\right] \frac{\left(\beta_{a}^{2}\right)^{n}}{n !} \\
& -\left[e^{-\beta_{a}^{2}} \sum_{n=1}^{\infty}[\psi(n+1)-\psi(1)] \frac{\left(\beta_{a}^{2}\right)^{n}}{n !}\right]^{2},
\end{aligned}
$$

con $\psi^{\prime}(1)=\pi^{2} / 6=1.644$. Para propósitos prácticos, también es posible mostrar la siguiente identidad 


$$
e^{-\beta^{2}} \sum_{n=1}^{\infty}[\psi(n+1)-\psi(1)] \frac{\left(\beta^{2}\right)^{n}}{n !}=-\sum_{n=1}^{\infty} \frac{(-1)^{n}}{n n !} z^{n},
$$

pero por otro lado se tiene también que $\sum_{n=1}^{\infty} \frac{(-1)^{n}}{n n !} z^{n}=-\left[E_{1}(z)-\right.$ $\psi(1)+\ln z]$ [41].

La estadística de los tiempos de paso 4.38 y 4.40 será utilizada para estudiar el criterio de detección de señales débiles.

\subsection{Detección de señales débiles}

Apliquemos ahora el criterio teórico para detectar señales débiles en el proceso de decaimiento del estado inestable de partículas activas. El criterio propuesto en términos de la estadística de los tiempos de paso es el mismo aplicado para el sistema láser, es decir

$$
\left[\langle t\rangle_{\beta}-\langle t\rangle_{\beta=0}\right]^{2} \geq(\Delta t)_{\beta=0}^{2} .
$$

De las ecs. 4.38 y 4.40 es claro que

$$
\left[\sum_{n=1}^{\infty} \frac{(-1)^{n}}{n n !} \beta_{a}^{2 n}\right]^{2} \geq \psi^{\prime}(1)
$$

La igualdad en esta ecuación nos permite determinar el valor crítico $\beta_{a c}$ del parámetro $\beta_{a}$ para el cual, en principio, es posible detectar la amplitud de la señal externa. Primeramente, calculamos el valor crítico en ausencia de actividad, con la ec. 4.43 y apoyandonos con Mathematica podemos resolver la ecuación donde si $K_{a}=1.0$ entonces $\beta_{a}^{2}=\beta_{0}^{2}$. En este caso se obtiene el valor crítico de $\beta_{0 c}=1.38429$. De acuerdo con el criterio, abajo de este valor no hay detección y por encima de dicho valor la detección se debe realizar de manera eficiente. En presencia de la actividad consideramos los siguientes valores de $K_{a}=1.5,2.0,2.5$, para los cuales de la ec. 4.43 se obtienen los valores críticos respectivos que son $\beta_{a c}=1.69541$, $\beta_{a c}=1.95769, \beta_{a c}=2.18876$. Lo que podemos corroborar de estos valores críticos es lo siguiente: para cada valor de $K_{a}$, se tiene que $\beta_{a c}=\sqrt{K_{a}} \beta_{0 c}$. 
Por otro lado, también podemos notar lo siguiente: si de la ec. 4.43, queremos calcular el valor crítico de $\beta_{a}$ sin introducir el parámetro $K_{a}$, obtenemos simplemente el valor $\beta_{a c}=1.38429$. Y si ahora tomamos en cuenta que $\beta_{a}^{2}=\frac{\beta_{0}^{2}}{k_{a}}$, de modo que $\beta_{a c}=\frac{\beta_{0 c}}{\sqrt{k_{a}}}=1.38429$. Pero recordemos que $\beta_{0}^{2}=\frac{F^{2}}{2 a D_{B}}$ y el valor crítico en ausencia de actividad es también $\beta_{0 c}=1.38429$ y entonces $\frac{F}{\sqrt{2 a D_{B}}}=1.38429$. De esta identidad podemos obtener el valor crítico de la amplitud de la fuerza eléctrica o del campo eléctrico por detectar en ausencia de actividad, esto es

$$
F_{\text {aus }}=\sqrt{2 a D_{B}} \beta_{0 c}=1.38429 \sqrt{2 a D_{B}} .
$$

Sin embargo, con actividad hemos obtenido el valor $\beta_{a c}=1.384$, y como $\beta_{a c}=\frac{\beta_{0 c}}{\sqrt{K_{a}}}=\frac{F}{\sqrt{K_{a}} \sqrt{2 a D_{B}}}$, entonces la fuerza eléctrica o campo eléctrico por detectar en este caso será

$$
F_{a c t}=\sqrt{K_{a}}\left[1.384 \sqrt{2 a D_{B}}\right]=\sqrt{K_{a}} F_{\text {aus }} .
$$

Si recordamos que la amplitud $F=\frac{q_{0} E_{0}}{\gamma_{v}}$, entonces la amplitud del campo eléctrico por detectar será

$$
E_{0}^{a c t}=\sqrt{K_{a}} E_{0}^{a u s}
$$

Estas mismas conclusiones se pueden obtener de la identidad $\beta_{a c}=\sqrt{K_{a}} \beta_{0 c}$ obtenida anteriormente, puesto que

$$
\beta_{a c}=\frac{\sqrt{K_{a}} F_{a u s}}{\sqrt{2 a D_{B}}}=\frac{F_{a c t}}{\sqrt{2 a D_{B}}},
$$

donde $F_{\text {act }}=\sqrt{K_{a}} F_{\text {aus }}$ y por consiguiente $E_{0}^{a c t}=\sqrt{K_{a}} E_{0}^{a u s}$.

Debido a que $K_{a}>1$ significa que en presencia de la actividad de las partículas la amplitud de la señal eléctrica sin actividad $E_{0}^{\text {aus }}$ se ve incrementada por el factor $\sqrt{K_{a}}$. En otras palabras, el valor de la señal se amplifica con la actividad de las partículas en su proceso de decaimiento.

Por otro lado, si aplicamos el criterio anterior en la región de $\beta_{a}$ muy pequeñas, en este caso el tiempo promedio de paso y la varianza se pueden aproximar por

$$
\langle 2 a t\rangle=\langle 2 a t\rangle_{0}-\beta_{a}^{2}
$$




$$
\left\langle(2 a \Delta t)^{2}\right\rangle=\psi^{\prime}(1) .
$$

Sustituyendo ambas ecuaciones en el criterio 4.42 obtenemos ahora el valor crítico

$$
\beta_{a c}=\left[\psi^{\prime}(1)\right]^{1 / 4}=1.132 .
$$

En este caso y similar a lo analizado anteriormente podemos concluir que

$$
F_{a c t}=\sqrt{K_{a}}\left[1.132 \sqrt{2 a D_{e}}\right]=\sqrt{K_{a}} F_{a u s},
$$

y para el campo eléctrico se tendrá $E_{0}^{a c t}=\sqrt{K_{a}} E_{0}^{a u s}$. Los valores críticos del parámetro $\beta_{a}$ en los casos exacto y aproximado, son representados en la siguiente tabla

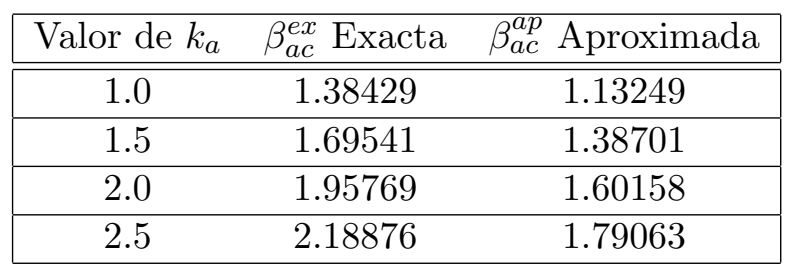

Tabla 4.1: Valores críticos del parámetro $\beta_{a}$ en los casos exacto y aproximado. Los valores $\beta_{0 c}^{e x}=1.38429$ y $\beta_{0 c}^{a p}=1.13249$, corresponden a los valores críticos exacto y aproximado en ausencia de actividad. 


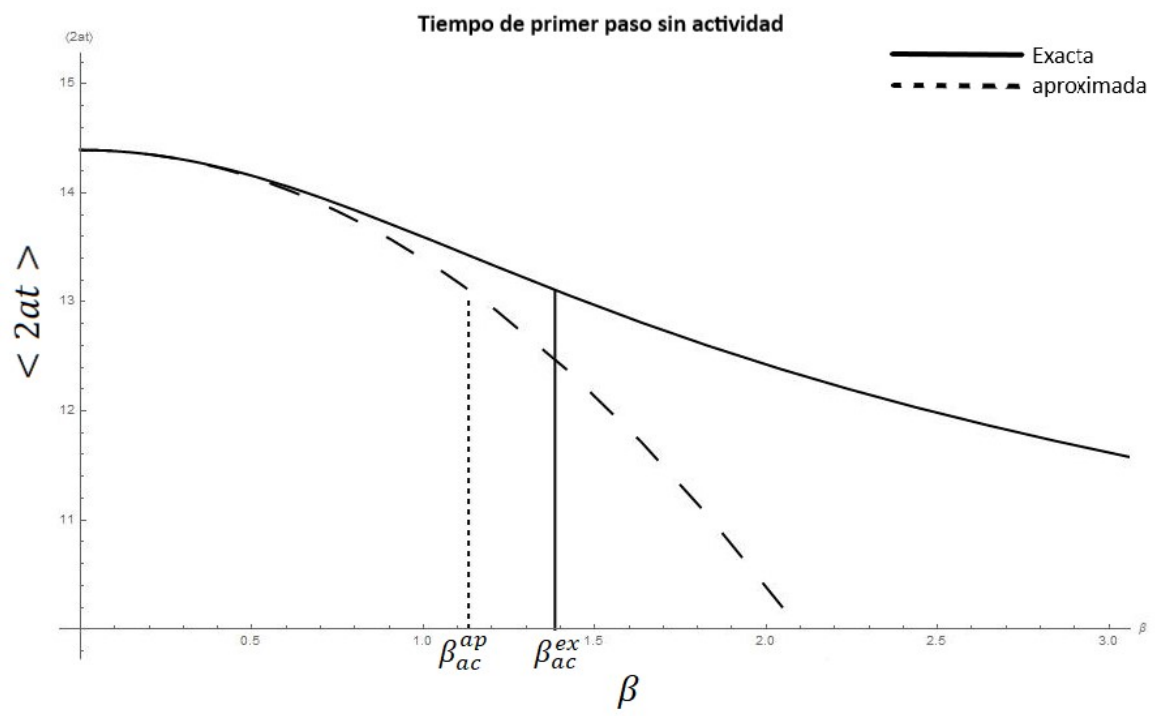

Figura 4.3: Comparación de los tiempos de primer paso sin actividad $K_{a}=1$. Caso exacto 4.38 (línea continua) y caso aproximado 4.48) (línea punteada). 


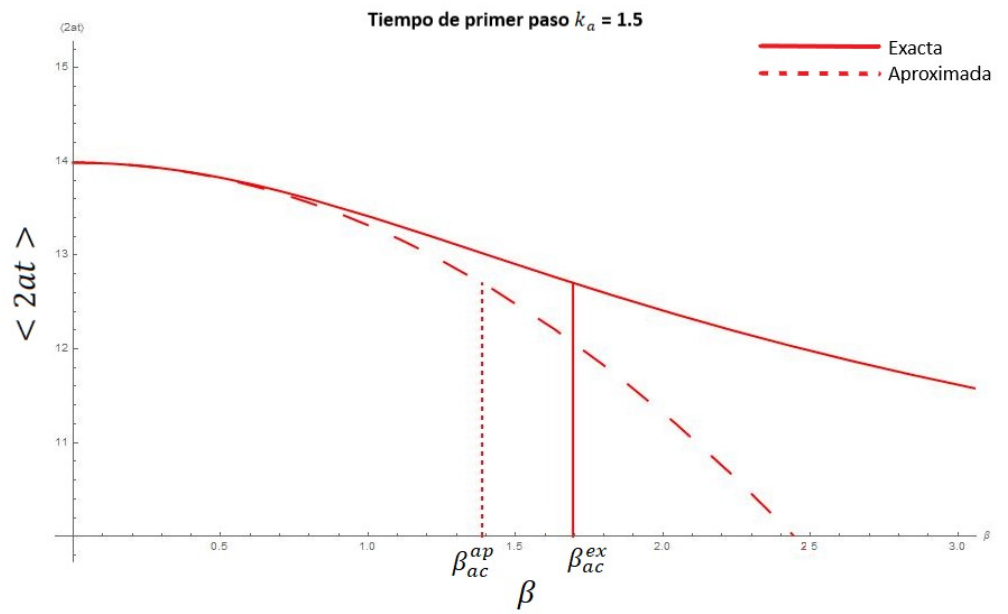

Figura 4.4: Comparación de los tiempos de primer paso con actividad $k_{a}=1.5$. Caso exacto 4.38 (línea continua) y caso aproximado 4.48) (línea punteada).

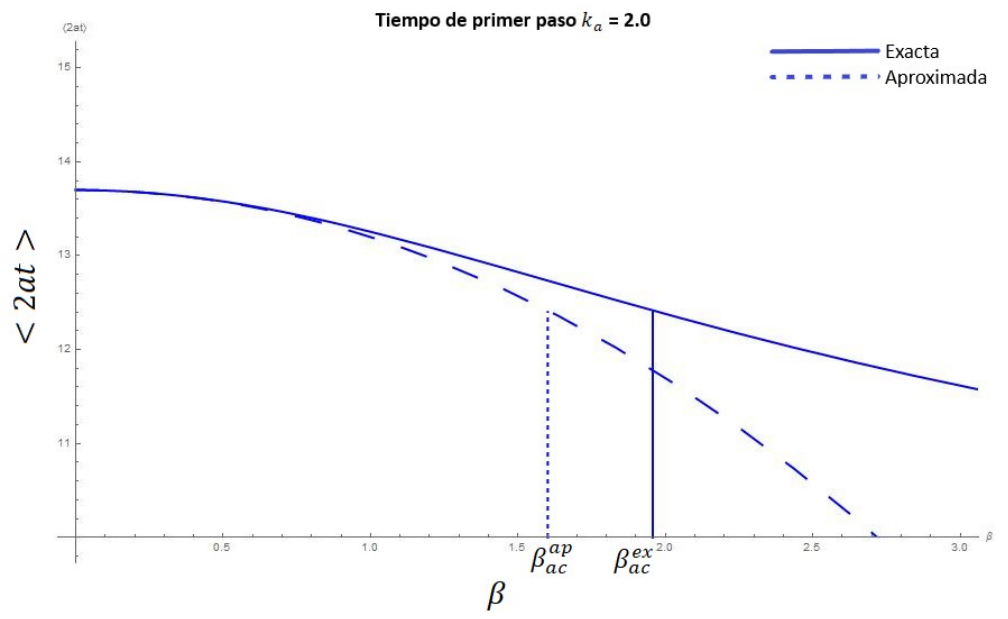

Figura 4.5: Comparación de los tiempos de primer paso con actividad $K_{a}=2.0$. Caso exacto 4.38 (línea continua) y caso aproximado 4.48 (línea punteada). 


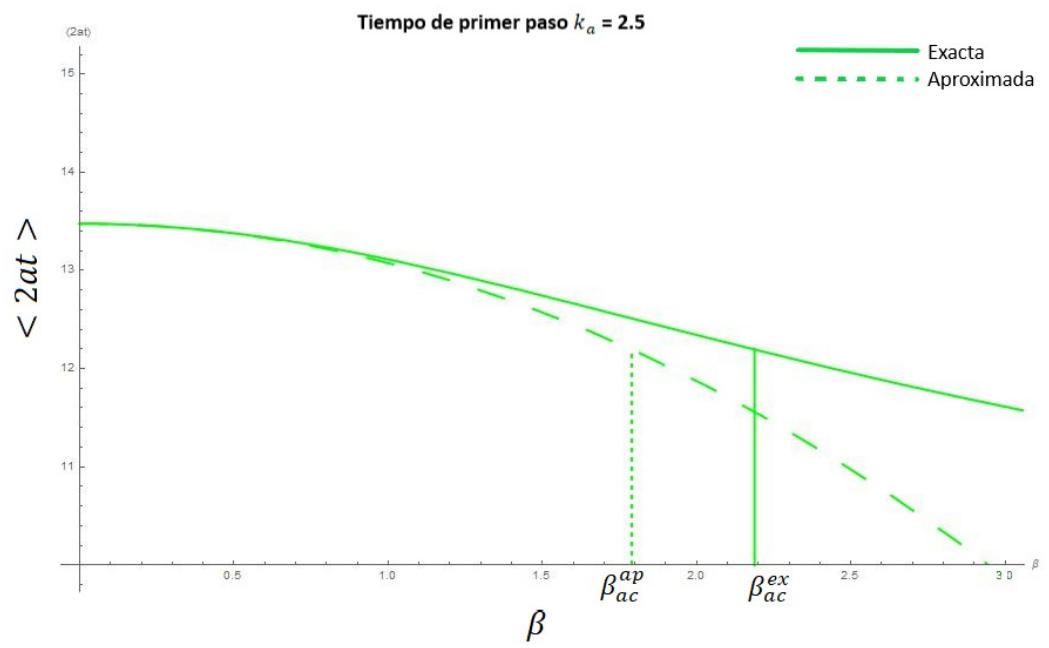

Figura 4.6: Comparación de los tiempos de primer paso con actividad $K_{a}=2.5$. Caso exacto 4.38 (línea continua) y caso aproximado 4.48 (línea punteada).

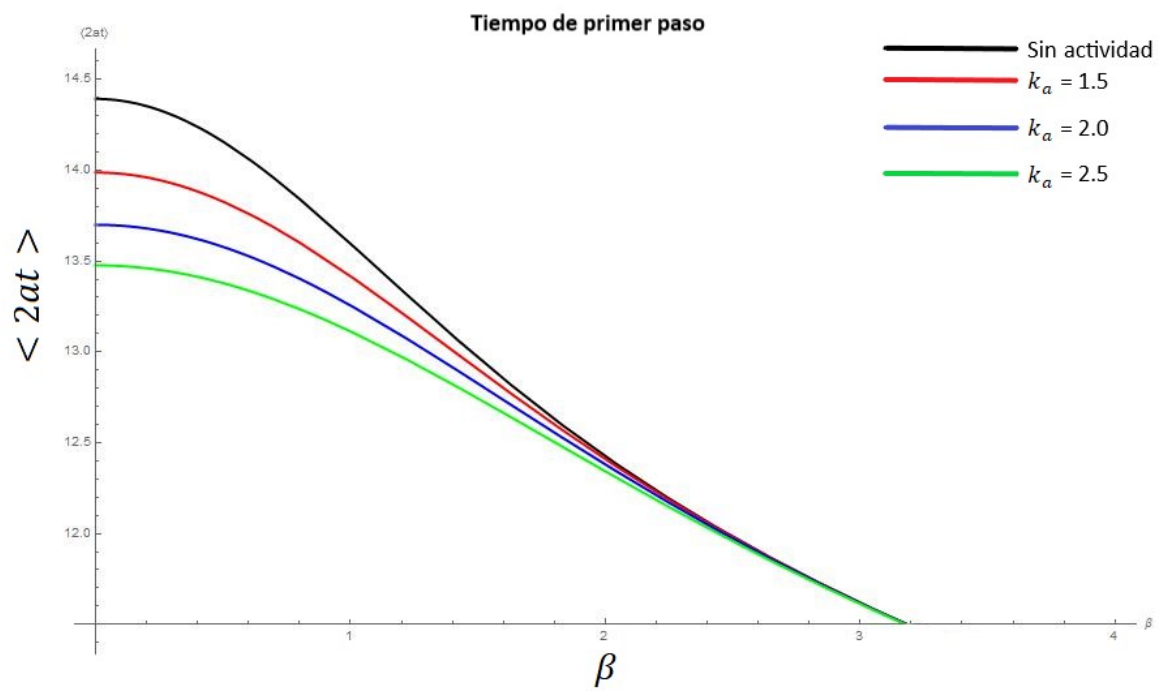

Figura 4.7: Comparación de los tiempos de primer paso exactos para distintos valores de $K_{a}$. 


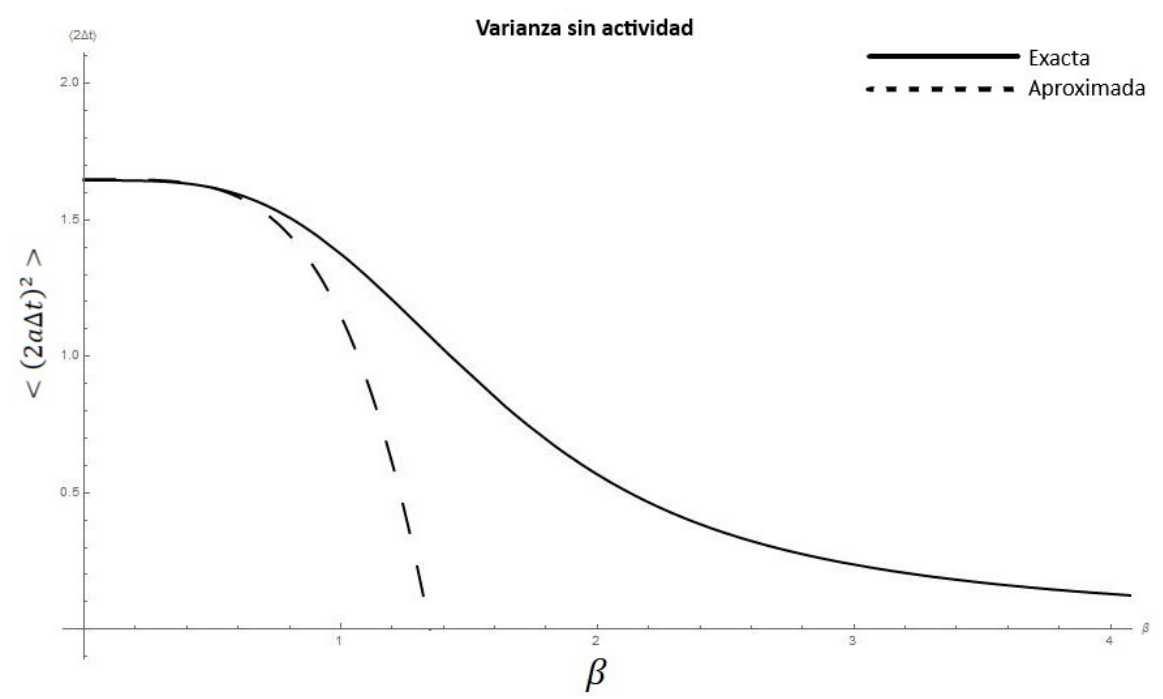

Figura 4.8: Comparación de la varianza sin actividad $k_{a}=1.0$. Caso exacto 4.40) (línea continua) y caso aproximado 4.49 (línea punteada).

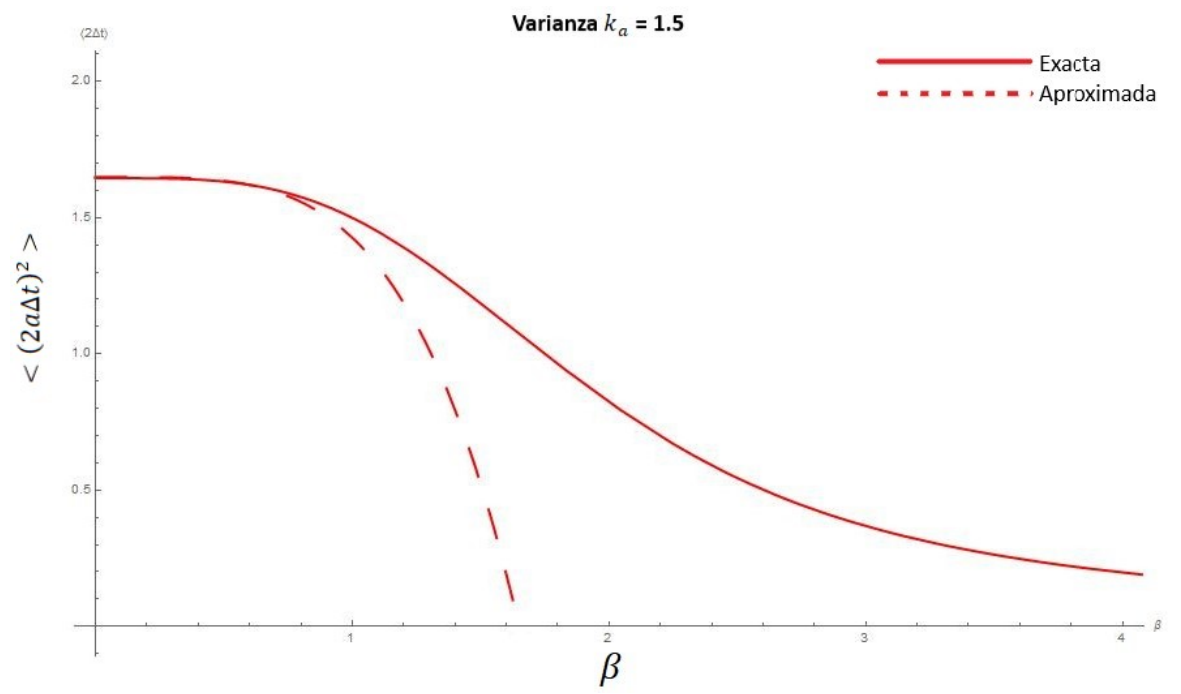

Figura 4.9: Comparación de la varianza con actividad $k_{a}=1.5$. Caso exacto (4.40) (línea continua) y caso aproximado 4.49 (línea punteada). 


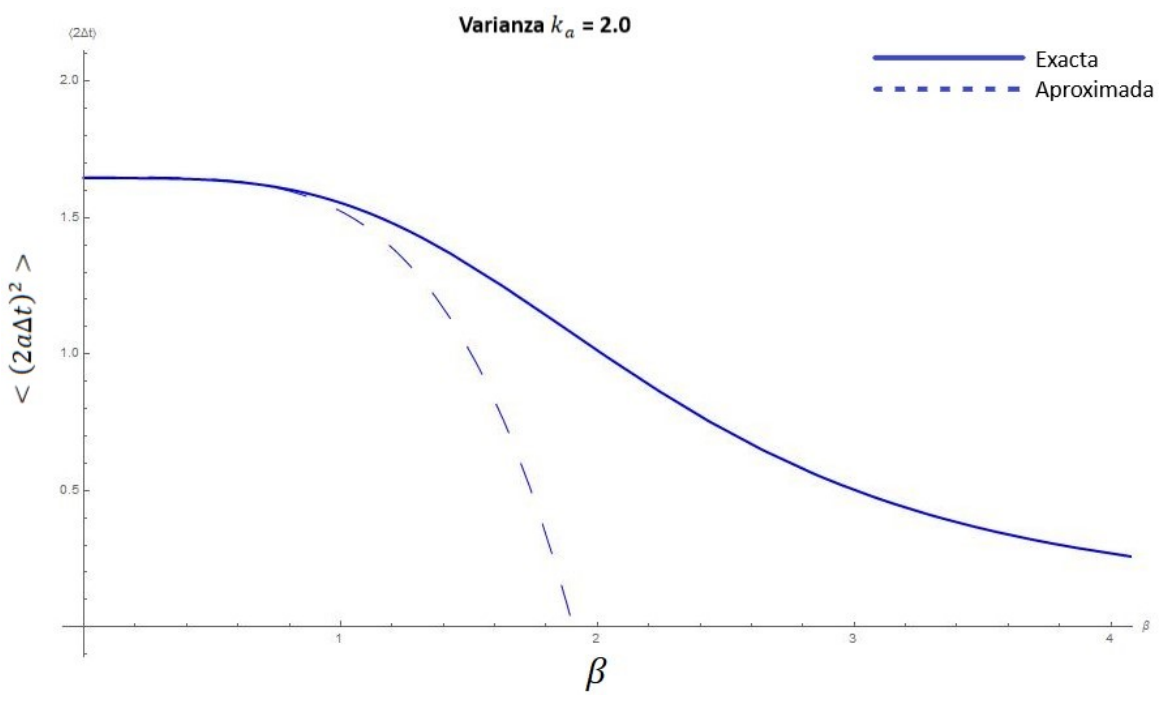

Figura 4.10: Comparación de la varianza con actividad $k_{a}=2.0$. Caso exacto 4.40 (línea continua) y caso aproximado 4.49 (línea punteada).

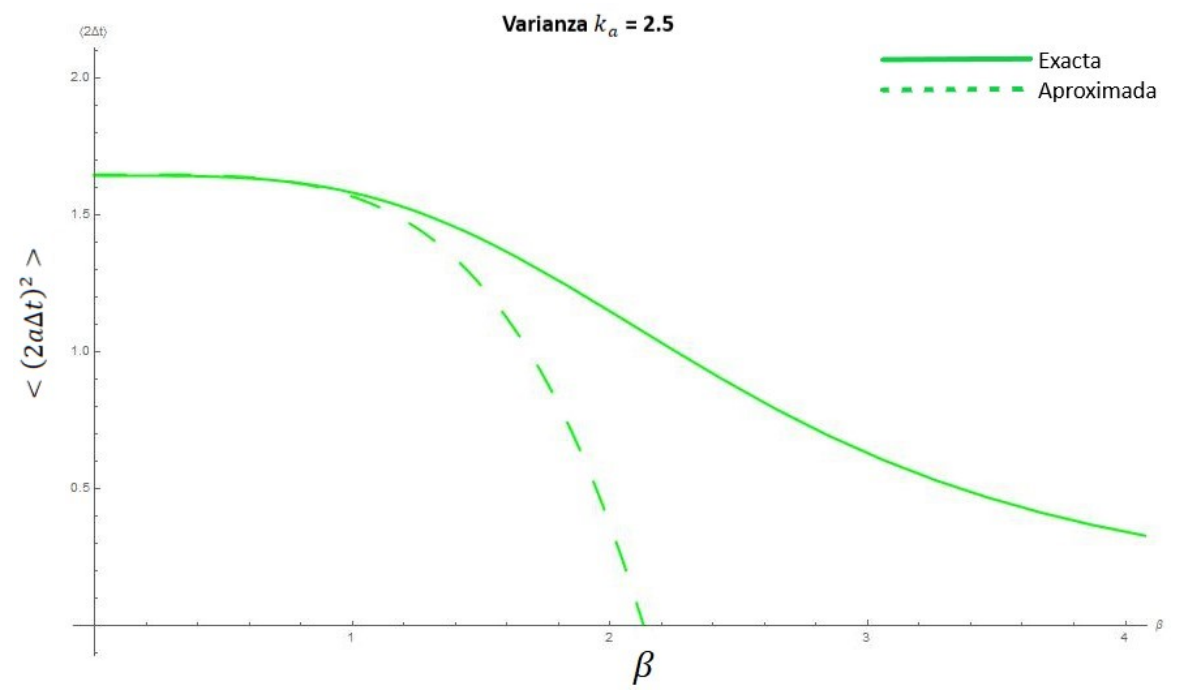

Figura 4.11: Comparación de la varianza con actividad $k_{a}=2.5$. Caso exacto 4.40) (línea continua) y caso aproximado 4.49) (línea punteada). 


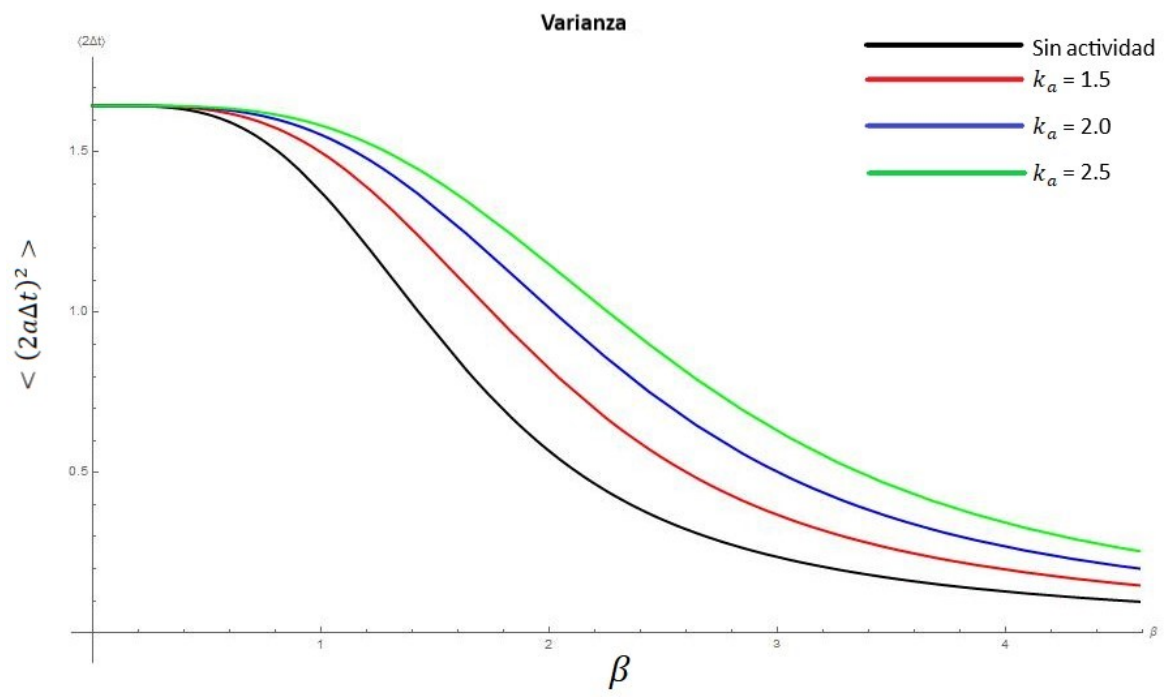

Figura 4.12: Comparación de las varianzas exactas para distintos valores de $K_{a}$. 


\section{Capítulo 5}

\section{Conclusiones}

En esta tesis hemos estudiado el proceso de detección de señales eléctricas débiles mediante un mecanismo alternativo al sistema láser, el cual consiste en la relajación dinámica del estado inestable de partículas activas (Janus) con carga eléctrica. Se ha utilizado el mismo criterio utilizado en el sistema láser relacionado con la estadística de los tiempos de primer paso. Debido al movimiento activo de las partículas brownianas se introduce otro grado de libertad a la ecuación de Langevin de 2D a través del ángulo azimutal $\varphi(t)$, permitiendo establecer un sistema acoplado de ecuaciones de Langevin; una ecuación está asociada al movimiento browniano convencional (movimiento pasivo) y la otra al movimiento browniano activo de rotación caracterizado por el ángulo azimutal mencionado.

El proceso de detección de señales débiles se lleva a cabo alrededor del estado inestable inicial, justo en un instante después de haberse desencadenado el decaimiento del estado inestable de la partícula activa por efecto del ruido interno (traslacional y rotacional). Por esta razón es suficiente considerar la aproximación lineal del potencial bidimensional imponiendo una barrera absorbente en la posición $R^{2}=k_{0} r_{s t}^{2}$, donde $r_{s t}^{2}$ es el valor del estado estacionario y $k_{0}$ es una constante lo suficientemente pequeña para garantizar que $R^{2} \ll r_{s t}^{2}$. Una vez establecidas estas condiciones, mostramos que la QDT permite caracterizar la relajación dinámica del estado inestable de manera exacta a través de la estadística de los tiempos de primer paso. 
Hemos mostrado que el criterio de detección es sensible a la señal externa a través del parámetro $\beta_{a}^{2}$, que su a vez contiene el efecto cooperativo entre la señal externa y el ruido interno traslacional y rotacional. Mediante dicho criterio hemos calculado los valores críticos del parámetro $\beta_{a}$ en ausencia y en presencia de la actividad de las partículas brownianas. En la tabla 4.1 se muestran los valores críticos de dicho parámetro de acuerdo con las expresiones exactas y aproximadas de la estadística de los tiempos de primer paso. En ambos casos se concluye que la amplitud de la fuerza eléctrica $F_{\text {aus }}$, que se detecta en ausencia de actividad, se ve incrementada en presencia de la actividad por el factor $\sqrt{K_{a}}>1$, es de$\operatorname{cir} F_{a c t}=\sqrt{K_{a}} F_{a u s}$, donde $F_{a u s}=\beta_{0 c} \sqrt{2 a D_{B}}, \beta_{0 c}$ puede ser $1.38429 \mathrm{o}$ 1.13249 , en los casos exacto y aproximado respectivamente.

En conclusión, cuando la amplitud de una señal externa débil ha sido detectada en el proceso de decaimiento del estado inestable de partículas brownianas pasivas, la amplitud de dicha señal será amplificada si dicho proceso de decaimiento se lleva a cabo con partículas brownianas activas. 


\section{Apéndice A}

\section{Función generadora y la estadística de los tiempos de paso.}

Para calcular la función generadora de momentos partimos de la expresión $G\left(2 \gamma_{1} \nu\right)=\left\langle\left(I_{r}^{\prime} / h^{\prime 2}\right)^{-\nu}\right\rangle$, tal que

$$
G\left(2 \gamma_{1} \nu\right)=\int_{0}^{\infty}\left(\frac{I_{r}^{\prime}}{h^{\prime 2}}\right)^{-\nu} P\left(h^{\prime}\right) d h^{\prime},
$$

donde $P\left(h^{\prime}\right)$ es la densidad de probabilidad marginal dada en la ec. (3.29), es decir

$$
P\left(h^{\prime}\right)=\frac{h^{\prime}}{\sigma^{2}} I_{0}\left(p^{\prime} h^{\prime} / \sigma^{2}\right) e^{-\left(1 / 2 \sigma^{2}\right)\left(h^{\prime 2}+p^{\prime 2}\right)^{2}} .
$$

Sustituyéndolo en la integral vemos que

$$
G\left(2 \gamma_{1} \nu\right)=2 \alpha^{2} I_{r}^{\prime^{-\nu}} e^{-\alpha^{2} p^{\prime 2}} \int_{0}^{\infty} h^{\prime^{2 \nu+1}} I_{0}\left(\alpha^{2} p^{\prime} h^{\prime}\right) e^{-\alpha^{2} h^{\prime^{2}}} d h^{\prime},
$$

siendo ahora $\alpha^{2}=\frac{1}{2 \sigma^{2}}$ y $\beta=\alpha p^{\prime}$. Con el cambio de variable $z=\alpha h^{\prime}$ tenemos que

$$
G\left(2 \gamma_{1} \nu\right)=2 \alpha^{-2 \nu} I_{r}^{\prime^{-\nu}} e^{-\frac{\beta^{2}}{2}} \underbrace{\int_{0}^{\infty} z^{2 \nu+1} I_{0}(\beta z) e^{-z^{2}} d z}_{A} .
$$


58péndice A. Función generadora y la estadística de los tiempos de paso.

Usamos ahora el desarrollo en serie de potencias de la función modificada de Bessel [41], de tal manera que la integral $A$ es igual a

$$
A=\sum_{n=0}^{\infty} \frac{\left(\beta^{2} / 2\right)^{n}}{\Gamma(n+1) n !} \int_{0}^{\infty} z^{2 \nu+2 n+1} e^{-z^{2}} d z .
$$

Después de evaluar la integral obtenemos entonces

$$
G\left(2 \gamma_{1} \nu\right)=\left(\frac{I_{r}^{\prime}}{2 \sigma^{2}}\right)^{-\nu} e^{-\frac{\beta^{2}}{2}} \sum_{n=0}^{\infty} \frac{\Gamma(\nu+n+1)}{(n !)^{2}}\left(\frac{\beta^{2}}{2}\right)^{n}
$$

Si tomamos en cuenta la función hipergeométrica confluente de Kummer [41] dada por

$$
M\left(\nu+1,1, \beta^{2} / 2\right)=\sum_{n=0}^{\infty} \frac{\Gamma(n+\nu+1)}{\Gamma(\nu+1)} \frac{1}{n !} \frac{\left(\beta^{2} / 2\right)^{n}}{n !},
$$

entonces la FGM también se puede escribir como

$$
G\left(2 \gamma_{1} \nu\right)=G_{0}\left(2 \gamma_{1} \nu\right) e^{-\beta^{2} / 2} M\left(\nu+1,1, \beta^{2} / 2\right),
$$

donde $G_{0}\left(2 \gamma_{1} \nu\right)=\left(I_{r}^{\prime} / 2 \sigma^{2}\right)^{-\nu} \Gamma(\nu+1)$.

\section{A.1. Estadística de los tiempos de paso}

La estadística de los tiempos de paso se obtiene mediante la FGM obtenida anteriormente, esto es

$$
\left\langle 2 \gamma_{1} t\right\rangle=-\left.\frac{d G}{d \nu}\right|_{\nu=0}, \quad\left\langle\left(2 \gamma_{1} t\right)^{2}\right\rangle=\left.\frac{d^{2} G}{d \nu^{2}}\right|_{\nu=0},
$$

Por simplicidad hacemos $G\left(2 \gamma_{1} \nu\right)=G_{0}\left(2 \gamma_{1} \nu\right) L(\nu)$ donde hemos definido a

$$
L(\nu)=e^{-\frac{\beta^{2}}{2}} \sum_{n=0}^{\infty} \frac{\Gamma(\nu+n+1)}{(n !)^{2}}\left(\frac{\beta^{2}}{2}\right)^{n},
$$

Tomando la primera derivada evaluada en cero, vamos a obtener 


$$
\left.\frac{d G}{d \nu}\right|_{\nu=0}=G_{0}^{\prime}(0) L(0)+G_{0}(0) L^{\prime}(0) .
$$

Calculando las derivadas correspondiente, obtenemos los siguientes resultados:

$$
\begin{aligned}
\left.G_{0}^{\prime}\left(a \gamma_{1} \nu\right)\right|_{\nu=0} & =-\ln \left(\frac{\gamma_{1} I_{r}^{\prime}}{\epsilon}\right)+\psi(1) \\
\left.L^{\prime}(\nu)\right|_{\nu=0} & =e^{-\frac{\beta^{2}}{2}} \sum_{n=0}^{\infty} \Gamma(n+1)[\psi(n+1)-\psi(1)] \frac{\left(\beta^{2} / 2\right)^{n}}{(n !)^{2}} \\
G_{0}(0) & =\Gamma(1)=1 \\
L(0) & =e^{-\frac{\beta^{2}}{2}} \sum_{n=0}^{\infty} \frac{\left(\beta^{2} / 2\right)^{n}}{n !}=1
\end{aligned}
$$

por lo que el primer momento será

$$
\left\langle 2 \gamma_{1} t\right\rangle=-G_{0}^{\prime}(0) L(0)-G_{0}(0) L^{\prime}(0) .
$$

Sustituyendo los resultados de las ec. A.12 vemos que el tiempo promedio de primer paso es de la forma

$$
\left\langle 2 \gamma_{1} t\right\rangle=\ln \left(\frac{\gamma_{1} I_{r}^{\prime}}{\epsilon}\right)-\psi(1)-e^{-\frac{\beta^{2}}{2}} \sum_{n=0}^{\infty}[\psi(n+1)-\psi(1)] \frac{\left(\beta^{2} / 2\right)^{n}}{n !},
$$

También es posible mostrar que

$$
e^{-\beta^{2}} \sum_{n=0}^{\infty}[\psi(n+1)-\psi(1)] \frac{\beta^{2 n}}{n !}=-\sum_{m=1}^{\infty} \frac{(-1)^{m}}{m m !}\left(\frac{\beta^{2}}{2}\right)^{m}
$$

y por lo tanto

$$
\left\langle 2 \gamma_{1} t\right\rangle=\left\langle 2 \gamma_{1} t\right\rangle_{0}+\sum_{m=1}^{\infty} \frac{(-1)^{m}}{m m !}\left(\frac{\beta^{2}}{2}\right)^{m}
$$

donde

$$
\left\langle 2 \gamma_{1} t\right\rangle_{0}=\ln \left(\frac{\gamma_{1} I_{r}^{\prime}}{\epsilon}\right)-\psi(1)
$$


6Apéndice A. Función generadora y la estadística de los tiempos de paso.

Para obtener el segundo momento calculamos la segunda derivada de la FGM, es decir

$$
\left.\frac{d^{2} G}{d \nu^{2}}\right|_{\nu=0}=G_{0}^{\prime \prime}(0) L(0)+2 G_{0}^{\prime}(0) L^{\prime}(0)+L^{\prime \prime}(0) G_{0}(0) .
$$

de tal manera que

$$
\begin{aligned}
\left.G_{0}^{\prime \prime}\left(a \gamma_{1} \nu\right)\right|_{\nu=0}= & \ln ^{2}\left(\frac{\gamma_{1} I_{r}^{\prime}}{\epsilon}\right)-2 \ln \left(\frac{\gamma I_{r}^{\prime}}{\epsilon}\right) \psi(1)+\psi^{2}(1)+\psi^{\prime}(1) \\
\left.L^{\prime \prime}(\nu)\right|_{\nu=0}= & e^{-\frac{\beta^{2}}{2}} \sum_{n=0}^{\infty} \Gamma(n+1) \frac{\left(\beta^{2} / 2\right)^{n}}{(n !)^{2}} \\
& {\left[\psi^{\prime}(n+1)-\psi^{\prime}(1)+(\psi(n+1)-\psi(1))^{2}\right], }
\end{aligned}
$$

y con los resultados obtenidos de las ec. A.12 y las derivadas anteriores tenemos ahora

$$
\left\langle\left(2 \gamma_{1} t\right)^{2}\right\rangle=G_{0}^{\prime \prime}(0)+2 G_{0}^{\prime}(0) L^{\prime}(0)+L^{\prime \prime}(0) .
$$

Para obtener la varianza definida, como $\left\langle\left(2 \gamma_{1} \Delta t\right)^{2}\right\rangle \equiv\left\langle\left(2 \gamma_{1} t\right)^{2}\right\rangle-\left\langle 2 \gamma_{1} t\right\rangle^{2}$, basta con sustituir lo que ya hemos calculado, es decir

$$
\left\langle\left(2 \gamma_{1} \Delta t\right)^{2}\right\rangle=G_{0}^{\prime \prime}(0)-G_{0}^{\prime 2}(0)+L^{\prime \prime}(0)-L^{\prime 2}(0) .
$$

Sustituyendo los valores correspondientes y haciendo un poco de álgebra podemos llegar a la ec. (3.35). 


\section{Apéndice B}

\section{Matriz de rotación}

Primero verificaremos que la matriz antisimétrica $W$ dada por

$$
W=\left(\begin{array}{ccc}
0 & \omega & 0 \\
-\omega & 0 & 0 \\
0 & 0 & 0
\end{array}\right)
$$

satisface la relación $e^{W t}=R e(t)$, donde

$$
R e(t)=\left(\begin{array}{ccc}
\cos \omega t & \sin \omega t & 0 \\
-\sin \omega t & \cos \omega t & 0 \\
0 & 0 & 1
\end{array}\right)
$$

Para tal propósito definimos el ángulo de rotación $\phi=\omega t$ y las matrices

$$
M_{z}=\left(\begin{array}{ccc}
0 & -i & 0 \\
i & 0 & 0 \\
0 & 0 & 0
\end{array}\right), \quad A=i M_{z}=\left(\begin{array}{ccc}
0 & 1 & 0 \\
-1 & 0 & 0 \\
0 & 0 & 0
\end{array}\right)
$$

de tal manera que $A$ es real y antisimétrica y por lo tanto $W t=i \phi M_{z}$. Usando la propiedad de la exponencial tenemos

$$
e^{W t}=e^{i \phi M_{Z}}=I+i \phi M_{z}+\frac{\left(i \phi M_{z}\right)^{2}}{2 !}+\frac{\left(i \phi M_{z}\right)^{3}}{3 !}+\cdots,
$$

donde I es la matriz unitaria. juntando los términos pares e impares y teniendo en cuenta que 


$$
M_{z}^{2 n}=S=\left(\begin{array}{ccc}
1 & 0 & 0 \\
0 & 1 & 0 \\
0 & 0 & 0
\end{array}\right), \quad M_{z}^{2 n+1}=M_{z},
$$

donde $\mathrm{S}$ es una matriz simétrica y $i M_{z}^{2 n+1}=A$, se puede mostrar que la Ec. (B.4) se reduce a

$$
e^{W t}=I+(\cos \phi-1) S+\sin \phi A,
$$

por lo tanto

$$
e^{W t}=\left(\begin{array}{ccc}
\cos \omega t & \sin \omega t & 0 \\
-\sin \omega t & \cos \omega t & 0 \\
0 & 0 & 1
\end{array}\right)
$$

La matriz anterior, es una matriz de rotación ortogonal porque $\operatorname{Re}(t) \operatorname{Re}^{T}(r)=$ $I$.

Por otro lado, de acuerdo con [46] Se muestra en general que cualquier matriz antisimétrica $W$ de $3 \times 3$ determina un vector de velocidad angular $\vec{\omega}$ de tal manera que $W=\vec{\omega} \times=\omega \mathbf{n} \times$, Donde $\mathbf{n}$ es un vector unitario a lo largo del eje de rotación. en este caso

$$
e^{W t}=e^{(\phi \mathbf{n} \times)}=I+\sum_{j=1}^{\infty} \frac{(\phi \mathbf{n} \times)^{j}}{j !} .
$$

La serie puede ser separada en potencias pares e impares de modo que

$$
e^{W t}=I+\sum_{j=1}^{\infty} \frac{(\phi \mathbf{n} \times)^{2 j}}{(2 j) !}+\sum_{j=0}^{\infty} \frac{(\phi \mathbf{n} \times)^{2 j+1}}{(2 j+1) !} .
$$

Siguiendo [46] la ecuación anterior puede ser escrita como

$$
e^{W t}=I+\sum_{j=1}^{\infty} \frac{(-1)^{j} \phi^{2 j}}{(2 j) !} S+\sum_{j=0}^{\infty} \frac{(-1)^{j} \phi^{2 j+1}}{(2 j+1) !} A
$$

donde $S=I-\mathbf{n n}^{T}$ y $A=\mathbf{n} \times$ son matrices simétricas y antisimétricas respectivamente. Ahora es claro que la última ecuación es de la forma 


$$
e^{W t}=I+(\cos \phi-1) S+\sin \phi A .
$$

En [47], se ha mostrado que cualquier matriz antisimétrica $W$ de $N \times N$ también satisface esa propiedad

$$
e^{W t}=I+\sum_{j=1}^{(N-C) / 2}\left\{\left(\cos \phi_{j}-1\right) S_{j}+\sin \phi_{j} A_{j}\right\},
$$

donde $\mathrm{C}$ es el número de eigenvectores reales linealmente independientes con eigenvalores cero; $S_{j}$ es simétrica y $A_{j}$ es antisimétrica. 


\section{Apéndice $\mathrm{C}$}

\section{Cálculo de $\left\langle e_{i}(t)\right\rangle$ y $\left\langle e_{i}(t) e_{j}\left(t^{\prime}\right)\right\rangle$}

\section{C.1. Valores promedio $\left\langle e_{i}(t)\right\rangle$}

Para calcular los valores promedio, hay que recordar que $\hat{\mathbf{e}}(t)=\left(e_{1}, e_{2}\right)=$ $(\cos \varphi, \sin \varphi)$, y por tanto

$$
\left\langle e_{1}(t)\right\rangle=\int_{-\infty}^{\infty} \cos \varphi P(\varphi, t) d \varphi
$$

donde $P(\varphi, t)$ está dado por

$$
P(\varphi, t)=\frac{1}{\sqrt{4 \pi D_{\Omega} t}} \exp \left[\frac{-\left(\varphi-\varphi_{0}\right)^{2}}{4 D_{\Omega} t}\right]
$$

así que

$$
\left\langle e_{1}(t)\right\rangle=\frac{1}{\sqrt{4 \pi D_{\Omega} t}} \int_{-\infty}^{\infty} \cos \varphi \exp \left[\frac{-\left(\varphi-\varphi_{0}\right)^{2}}{4 D_{\Omega} t}\right] d \varphi .
$$

Si definimos como $A=\frac{1}{4 D_{\Omega} t}$ y utilizamos el cambio de variable $u=\varphi-\varphi_{0}$, entonces

$$
\left\langle e_{1}(t)\right\rangle=\sqrt{\frac{A}{\pi}} \int_{-\infty}^{\infty} \cos \left(u+\varphi_{0}\right) e^{-A u^{2}} d u
$$

Escribiendo la función coseno en término de exponenciales, tenemos ahora

$$
\left\langle e_{1}(t)\right\rangle=\frac{1}{2} \sqrt{\frac{A}{\pi}}\left\{\int_{-\infty}^{\infty} e^{i\left(u+\varphi_{0}\right)-A u^{2}} d u+\int_{-\infty}^{\infty} e^{-i\left(u+\varphi_{0}\right)-A u^{2}} d u\right\},
$$


completando el cuadrado en los exponentes es fácil verificar que

$$
\begin{aligned}
i\left(u+\varphi_{0}\right)-A u^{2} & =-A\left(u-\frac{i}{2 A}\right)^{2}+i \varphi_{0}-\frac{1}{4 A}, \\
-i\left(u+\varphi_{0}\right)-A u^{2} & =-A\left(u+\frac{i}{2 A}\right)^{2}-i \varphi_{0}-\frac{1}{4 A},
\end{aligned}
$$

de manera que entonces

$$
\left\langle e_{1}(t)\right\rangle=\frac{1}{2} \sqrt{\frac{A}{\pi}} e^{-\frac{1}{4 A}}\left[e^{i \varphi_{0}} \int_{-\infty}^{\infty} e^{-A\left(u-\frac{i}{2 A}\right)^{2}} d u+e^{-i \varphi_{0}} \int_{-\infty}^{\infty} e^{-A\left(u+\frac{i}{2 A}\right)^{2}} d u\right],
$$

de donde concluimos que

$$
\left\langle e_{1}(t)\right\rangle=e^{-D_{\Omega} t} \cos \varphi_{0},
$$

el cual coincide con el resultado mostrado en la ec.4.23. El cálculo del promedio $\left\langle e_{2}(t)\right\rangle$ es enteramente análogo.

\section{C.2. Funciones de correlación $\left\langle e_{i}(t) e_{j}\left(t^{\prime}\right)\right\rangle$}

Cálculo de las funciones de correlación a partir de la ec. 4.12).

Cálculo de la función de correlación $\left\langle e_{1}(t) e_{1}\left(t^{\prime}\right)\right\rangle$. En este caso $i=j=1$ y para tiempos tales que $t>t^{\prime}$, se tiene que

$$
\begin{aligned}
\left\langle e_{1}(t) e_{1}\left(t^{\prime}\right)\right\rangle= & \int d \varphi \int d \varphi^{\prime} \cos \varphi \cos \varphi^{\prime} \frac{1}{\sqrt{4 \pi D_{\Omega}\left(t-t^{\prime}\right)}} \exp \left[-\frac{\left(\varphi-\varphi^{\prime}\right)^{2}}{4 D_{\Omega}\left(t-t^{\prime}\right)}\right] \\
& \frac{1}{\sqrt{4 \pi D_{\Omega} t^{\prime}}} \exp \left[-\frac{\left(\varphi^{\prime}-\varphi_{0}\right)^{2}}{4 D_{\Omega} t^{\prime}}\right] .
\end{aligned}
$$

Si definimos los siguientes parámetros

$$
A=\frac{1}{4 D_{\Omega}\left(t-t^{\prime}\right)}, \quad B=\frac{1}{4 D_{\Omega} t^{\prime}},
$$


entonces la ecuación anterior la podemos escribir como sigue

$$
\left\langle e_{1}(t) e_{1}\left(t^{\prime}\right)\right\rangle=\sqrt{\frac{A}{\pi}} \sqrt{\frac{B}{\pi}} \int d \varphi \int d \varphi^{\prime} \cos \varphi \cos \varphi^{\prime} e^{-A\left(\varphi-\varphi^{\prime}\right)^{2}} e^{-B\left(\varphi^{\prime}-\varphi_{0}\right)^{2}} .
$$

Integramos primero en la variable $\varphi$ introduciendo el cambio de variable $u=\varphi-\varphi^{\prime}$ tal que $\varphi=u+\varphi^{\prime}$ y $d \varphi=d u$. En este caso la correlación es ahora

$$
\left\langle e_{1}(t) e_{1}\left(t^{\prime}\right)\right\rangle=\frac{\sqrt{A B}}{\pi} \int d \varphi^{\prime} \cos \varphi^{\prime} e^{-B\left(\varphi^{\prime}-\varphi_{0}\right)^{2}} \underbrace{\int d u \cos \left(u+\varphi^{\prime}\right) e^{-A u^{2}}}_{I_{1}},
$$

sin embargo, $I_{1}$ se puede escribir como

$$
I_{1}=\int d u\left[\frac{1}{2}\left(e^{i\left(u+\varphi^{\prime}\right)}+e^{-i\left(u+\varphi^{\prime}\right)}\right)\right] e^{-A u^{2}} .
$$

Por otro lado, es fácil mostrar que

$$
\begin{aligned}
i\left(u+\varphi^{\prime}\right)-A u^{2} & =-A\left(u-\frac{i}{2 A}\right)^{2}+i \varphi^{\prime}-\frac{1}{4 A}, \\
-i\left(u+\varphi^{\prime}\right)-A u^{2} & =-A\left(u+\frac{i}{2 A}\right)^{2}-i \varphi^{\prime}-\frac{1}{4 A},
\end{aligned}
$$

luego entonces

$$
I_{1}=\frac{1}{2}\left\{\int_{-\infty}^{\infty} d u e^{-A\left(u-\frac{i}{2 A}\right)^{2}+i \varphi^{\prime}-\frac{1}{4 A}}+\int_{-\infty}^{\infty} d u e^{-A\left(u+\frac{i}{2 A}\right)^{2}-i \varphi^{\prime}-\frac{1}{4 A}}\right\},
$$

o bien

$$
\begin{aligned}
I_{1} & =\frac{1}{2} e^{-\frac{1}{4 A}}\left[e^{i \varphi^{\prime}}\left(\sqrt{\frac{\pi}{A}}\right)+e^{-i \varphi^{\prime}}\left(\sqrt{\frac{\pi}{A}}\right)\right] \\
& =\sqrt{\frac{\pi}{A}} e^{-\frac{1}{4 A}} \cos \varphi^{\prime} .
\end{aligned}
$$

La función de correlación se reduce ahora a la siguiente integral

$$
\begin{aligned}
\left\langle e_{1}(t) e_{1}\left(t^{\prime}\right)\right\rangle & =\frac{\sqrt{A B}}{\pi} \int d \varphi^{\prime} \cos \varphi^{\prime} e^{-B\left(\varphi^{\prime}-\varphi_{0}\right)^{2}}\left(\sqrt{\frac{\pi}{A}} e^{-\frac{1}{4 A}} \cos \varphi^{\prime}\right) \\
& =\sqrt{\frac{B}{\pi}} e^{-\frac{1}{4 A}} \int d \varphi^{\prime} \cos ^{2} \varphi^{\prime} e^{-B\left(\varphi^{\prime}-\varphi_{0}\right)^{2}} .
\end{aligned}
$$


Para evaluar esta integral, introducimos de nueva cuenta el cambio de variable $z=\varphi^{\prime}-\varphi_{0}$, y algunas de la identidades trigonométricas conocidas para mostrar que

$$
\left\langle e_{1}(t) e_{1}\left(t^{\prime}\right)\right\rangle=\frac{1}{2} \sqrt{\frac{B}{\pi}} e^{-\frac{1}{4 A}} \underbrace{\int d z\left(1+\cos 2\left(z+\varphi_{0}\right)\right) e^{-B z^{2}}}_{I_{2}} .
$$

Pero $I_{2}$ se puede escribir como

$$
I_{2}=\int d z e^{-B z^{2}}+\int d z\left(\frac{e^{2 i\left(z+\varphi_{0}\right)}+e^{-2 i\left(z+\varphi_{0}\right)}}{2}\right) e^{-B z^{2}},
$$

y completando los cuadrados se tiene que

$$
\begin{aligned}
2 i\left(z+\varphi_{0}\right)-B z^{2} & =-B\left(z-\frac{i}{B}\right)^{2}+2 i \varphi_{0}-\frac{1}{B} \\
-2 i\left(z+\varphi_{0}\right)-B z^{2} & =-B\left(z+\frac{i}{B}\right)^{2}-2 i \varphi_{0}-\frac{1}{B}
\end{aligned}
$$

Al sustituir estas expresiones y evaluando la integración correspondiente obtenemos

$$
\begin{aligned}
I_{2} & =\sqrt{\frac{\pi}{B}}+\frac{1}{2} e^{-1 / B}\left(\sqrt{\frac{\pi}{B}} e^{2 i \varphi_{0}}+\sqrt{\frac{\pi}{B}} e^{-2 i \varphi_{0}}\right) \\
& =\sqrt{\frac{\pi}{B}}\left(1+e^{-1 / B} \cos \left(2 \varphi_{0}\right)\right) .
\end{aligned}
$$

Finalmente sustituyendo los valores de $A, B$ y $I_{2}$ en la función de correlación obtenemos que

$$
\left\langle e_{1}(t) e_{1}\left(t^{\prime}\right)\right\rangle=\frac{1}{2} e^{-D_{\Omega}\left(t-t^{\prime}\right)}\left[1+\cos \left(2 \varphi_{0}\right) e^{-4 D_{\Omega} t^{\prime}}\right] \quad t>t^{\prime}
$$

que es precisamente el resultado expresado en la ec. (4.13). Es fácil mostrar que $\left\langle e_{1}(t) e_{1}\left(t^{\prime}\right)\right\rangle=\left\langle e_{2}(t) e_{2}\left(t^{\prime}\right)\right\rangle$. 
Cálculo de la función de correlación $\left\langle e_{1}(t) e_{2}\left(t^{\prime}\right)\right\rangle$. En este caso $i=1$ y $j=2$, y por tanto

$$
\left\langle e_{1}(t) e_{2}\left(t^{\prime}\right)\right\rangle=\sqrt{\frac{A}{\pi}} \sqrt{\frac{B}{\pi}} \int d \varphi \int d \varphi^{\prime} \cos \varphi \sin \varphi^{\prime} e^{-A\left(\varphi-\varphi^{\prime}\right)^{2}} e^{-B\left(\varphi^{\prime}-\varphi_{0}\right)^{2}} .
$$

Siguiendo los pasos algebraicos muy similares al caso anterior, se puede mostrar que

$$
\left\langle e_{1}(t) e_{2}\left(t^{\prime}\right)\right\rangle=\frac{\sqrt{A B}}{\pi} \int d \varphi^{\prime} \sin \varphi^{\prime} e^{-B\left(\varphi^{\prime}-\varphi_{0}\right)^{2}} \underbrace{\int d u \cos \left(u+\varphi^{\prime}\right) e^{-A u^{2}}}_{L_{1}},
$$

donde en este caso

$$
L_{1}=\frac{1}{2}\left\{\int d u e^{-A\left(u-\frac{i}{2 A}\right)^{2}+i \varphi^{\prime}-\frac{1}{4 A}}+\int d u e^{-A\left(u+\frac{i}{2 A}\right)^{2}-i \varphi^{\prime}-\frac{1}{4 A}}\right\},
$$

de modo que

$$
L_{1}=\sqrt{\frac{\pi}{A}} e^{-\frac{1}{4 A}} \cos \varphi^{\prime}
$$

y la función de correlación es ahora

$$
\left\langle e_{1}(t) e_{2}\left(t^{\prime}\right)\right\rangle=\sqrt{\frac{B}{\pi}} e^{-\frac{1}{4 A}} \underbrace{\int d \varphi^{\prime} \sin \varphi^{\prime} \cos \varphi^{\prime} e^{-B\left(\varphi^{\prime}-\varphi_{0}\right)^{2}}}_{L_{2}} .
$$

De forma similar, introducimos el cambio de variable $z=\varphi^{\prime}-\varphi_{0}$. En este caso

$$
L_{2}=\frac{1}{2} \int d z\left(\frac{e^{2 i\left(z+\varphi_{0}\right)}-e^{-2 i\left(z+\varphi_{0}\right)}}{2 i}\right) e^{-B z^{2}},
$$

y completando el cuadrado en los exponentes tenemos

$$
\begin{aligned}
2 i\left(z+\varphi_{0}\right)-B z^{2} & =-B\left(z-\frac{i}{B}\right)^{2}+2 i \varphi_{0}-\frac{i}{B} \\
-2 i\left(z+\varphi_{0}\right)-B z^{2} & =-B\left(z+\frac{i}{B}\right)^{2}-2 i \varphi_{0}-\frac{i}{B},
\end{aligned}
$$


sustituyendo y realizando la integración se muestra que

$$
L_{2}=\frac{1}{2} \sqrt{\frac{\pi}{B}} e^{1 / B} \sin \left(2 \varphi_{0}\right)
$$

Obteniendo finalmente el resultado

$$
\left\langle e_{1}(t) e_{2}\left(t^{\prime}\right)\right\rangle=\frac{1}{2} e^{-D_{\Omega}\left(t-t^{\prime}\right)}\left[\sin \left(2 \varphi_{0}\right) e^{-4 D_{\Omega} t^{\prime}}\right] \quad t>t^{\prime} .
$$

También $\left\langle e_{1}(t) e_{2}\left(t^{\prime}\right)\right\rangle=\left\langle e_{1}\left(t^{\prime}\right) e_{2}(t)\right\rangle$. 


\section{Apéndice D}

\section{Cálculo de la matriz $\sigma_{i j}$}

De acuerdo con la ec. 4.25 la matriz $\sigma_{i j}$ está dada por

$$
\begin{aligned}
\sigma_{i j}=\int_{0}^{\infty} \int_{0}^{\infty} e^{-a\left(t+t^{\prime}\right)} & \left\langle f_{i}(t) f_{j}\left(t^{\prime}\right)\right\rangle d t d t^{\prime} \\
& +U_{s}^{2} \int_{0}^{\infty} \int_{0}^{\infty} e^{-a\left(t+t^{\prime}\right)}\left\langle e_{i}(t) e_{j}\left(t^{\prime}\right)\right\rangle d t d t^{\prime} \\
& \quad-U_{s}^{2} \int_{0}^{\infty} e^{-a t}\left\langle e_{i}(t)\right\rangle d t \int_{0}^{\infty} e^{-a t^{\prime}}\left\langle e_{j}\left(t^{\prime}\right)\right\rangle d t^{\prime} .
\end{aligned}
$$

Cálculo de $\sigma_{11}$ con $i=j=1$. Definimos el primer término de (D.1) como

$$
\begin{aligned}
I_{a}=\int_{0}^{\infty} \int_{0}^{\infty} e^{-a\left(t+t^{\prime}\right)}\left\langle f_{1}(t) f_{1}\left(t^{\prime}\right)\right\rangle d t d t^{\prime} & \\
=2 D_{B} \int_{0}^{\infty} \int_{0}^{\infty} e^{-a\left(t+t^{\prime}\right)} \delta\left(t-t^{\prime}\right) d t d t^{\prime} & =2 D_{B} \int_{0}^{\infty} e^{-2 a t} d t=\frac{D_{B}}{a} .
\end{aligned}
$$

Para el segundo término de (D.1) definimos ahora la doble integral como

$$
I_{b}=U_{s}^{2} \int_{0}^{\infty} \int_{0}^{\infty} e^{-a\left(t+t^{\prime}\right)}\left\langle e_{1}(t) e_{1}\left(t^{\prime}\right)\right\rangle d t d t^{\prime} .
$$


Sustituyendo la expresión de la función de correlación obtenida en el apéndice anterior para $t>t^{\prime}$, tenemos entonces

$$
I_{b}=\frac{U_{s}^{2}}{2} \int_{0}^{\infty} \int_{0}^{\infty} e^{-a\left(t+t^{\prime}\right)}\left[e^{-D_{\Omega}\left(t-t^{\prime}\right)}\left(1+\cos \left(2 \varphi_{0}\right) e^{-4 D_{\Omega} t^{\prime}}\right)\right] d t d t^{\prime} .
$$

Reacomodando los términos las integrales resultantes son

$$
\begin{aligned}
& I_{b}=\frac{U_{s}^{2}}{2}(\underbrace{\int_{0}^{\infty} e^{-\left(a+D_{\Omega}\right) t} d t \int_{0}^{t} e^{-\left(a-D_{\Omega}\right) t^{\prime}} d t^{\prime}}_{J_{1}} \\
& +\cos \left(2 \varphi_{0}\right) \underbrace{\int_{0}^{\infty} e^{-\left(a+D_{\Omega}\right) t} d t \int_{0}^{t} e^{-\left(a+3 D_{\Omega}\right) t^{\prime}} d t^{\prime}}_{J_{2}}) .
\end{aligned}
$$

Evaluando las integrales obtenemos primero para $J_{1}$

$$
\begin{aligned}
J_{1} & =\int_{0}^{\infty} e^{-\left(a+D_{\Omega}\right) t} d t\left[\frac{1}{\left(a-D_{\Omega}\right)}\left(1-e^{-\left(a-D_{\Omega}\right) t}\right)\right] \\
& =\frac{1}{\left(a-D_{\Omega}\right)}\left(\int_{0}^{\infty} e^{-\left(a+D_{\Omega}\right) t} d t-\int_{0}^{\infty} e^{-2 a t} d t\right),
\end{aligned}
$$

luego entonces

$$
J_{1}=\frac{1}{2 a\left(a+D_{\Omega}\right)}
$$

Para $J_{2}$ tenemos que

$$
J_{2}=\cos \left(2 \varphi_{0}\right) \int_{0}^{\infty} e^{-\left(a+D_{\Omega}\right) t} d t\left[\frac{1}{\left(a+3 D_{\Omega}\right)}\left(1-e^{-\left(a+3 D_{\Omega}\right) t}\right)\right],
$$

por lo que

$$
J_{2}=\frac{\cos \left(2 \varphi_{0}\right)}{\left(a+3 D_{\Omega}\right)}\left(\int_{0}^{\infty} e^{-\left(a+D_{\Omega}\right) t} d t-\int_{0}^{\infty} e^{-\left(2 a+4 D_{\Omega}\right) t} d t\right),
$$

y por tanto

$$
J_{2}=\frac{\cos \left(2 \varphi_{0}\right)}{2\left(a+D_{\Omega}\right)\left(a+2 D_{\Omega}\right)}
$$


Así que sumando $J_{1}$ y $J_{2}$ concluimos que

$$
I_{b}=\frac{U_{s}^{2}}{2}\left[\frac{1}{2 a\left(a+D_{\Omega}\right)}+\frac{\cos \left(2 \varphi_{0}\right)}{2\left(a+D_{\Omega}\right)\left(a+2 D_{\Omega}\right)}\right] .
$$

Es importante resaltar que el resultado (D.7) se ha obtenido para el caso en que $t>t^{\prime}$. Si $t^{\prime}<t$, entonces en la función de correlación $\left\langle e_{1}(t) e_{1}\left(t^{\prime}\right)\right\rangle$ dada en la ec.4.13, los tiempos $t$ y $t^{\prime}$ intercambian sus posiciones. En este caso se puede demostrar que $I_{b}$ conduce al mismo resultado que (D.7) y por lo tanto este resultado debe estar multiplicado por el factor 2 , es decir, $2 I_{b}$.

El tercer término de (D.1) lo definimos como

$$
\begin{aligned}
I_{c} & =U_{s}^{2} \int_{0}^{\infty} e^{-a t}\left\langle e_{1}(t)\right\rangle d t \int_{0}^{\infty} e^{-a t^{\prime}}\left\langle e_{1}\left(t^{\prime}\right)\right\rangle d t^{\prime} \\
& =U_{s}^{2}\left(\int_{0}^{\infty} e^{-a t}\left\langle e_{1}(t)\right\rangle d t\right)^{2},
\end{aligned}
$$

realizando la integración concluimos que este término se puede escribir como

$$
I_{c}=\frac{U_{s}^{2}}{\left(a+D_{\Omega}\right)^{2}} \frac{1}{2}\left[1+\cos \left(2 \varphi_{0}\right)\right] .
$$

Finalmente el elemento de matriz $\sigma_{11}=I_{a}+2 I_{b}-I_{c}$, y por lo tanto

$$
\sigma_{11}=\frac{D_{B}}{a}+\frac{U_{s}^{2} D_{\Omega}}{2 a\left(a+D_{\Omega}\right)^{2}}-\frac{U_{s}^{2} D_{\Omega} \cos \left(2 \varphi_{0}\right)}{2\left(a+D_{\Omega}\right)^{2}\left(a+2 D_{\Omega}\right)} .
$$

Cálculo de la $\sigma_{12}$. En este caso el primer término lo definimos como

$$
L_{a}=\int_{0}^{\infty} \int_{0}^{\infty} e^{-a\left(t+t^{\prime}\right)}\left\langle f_{1}(t) f_{2}\left(t^{\prime}\right)\right\rangle d t d t^{\prime}=0,
$$

el segundo término lo definimos como

$$
L_{b}=U_{s}^{2} \int_{0}^{\infty} \int_{0}^{\infty} e^{-a\left(t+t^{\prime}\right)}\left\langle e_{1}(t) e_{2}\left(t^{\prime}\right)\right\rangle d t d t^{\prime},
$$


sustituimos ahora la función de correlación correspondiente, tomando en cuenta que $t>t^{\prime}$, es decir

$$
L_{b}=\frac{U_{s}^{2}}{2} \sin \left(2 \varphi_{0}\right) \int_{0}^{\infty} e^{-\left(a+D_{\Omega}\right) t} d t \int_{0}^{t} e^{-\left(a+3 D_{\Omega} t^{\prime}\right)} d t^{\prime},
$$

la cual es una integral que ya habíamos resuelto por lo que el resultado es inmediato

$$
L_{b}=\frac{U_{s}^{2}}{2} \sin \left(2 \varphi_{0}\right)\left(\frac{1}{2\left(a+D_{\Omega}\right)\left(a+2 D_{\Omega}\right)}\right) .
$$

De igual manera si $t^{\prime}>t$, se obtiene el mismo resultado como en (D.12) y por tanto $L_{b}$ debe ir multiplicado por el factor 2 .

Para el tercer término de $\sigma_{12}$ se tiene que

$$
L_{c}=U_{s}^{2} \int_{0}^{\infty} e^{-a t}\left\langle e_{1}(t)\right\rangle d t \int_{0}^{\infty} e^{-a t^{\prime}}\left\langle e_{2}\left(t^{\prime}\right)\right\rangle d t^{\prime},
$$

sustituimos ahora las expresiones de los valores promedio para obtener

$$
L_{c}=U_{s}^{2} \cos \left(\varphi_{0}\right) \sin \left(\varphi_{0}\right)\left(\int_{0}^{\infty} e^{-\left(a+D_{\Omega}\right) t} d t\right)^{2},
$$

de donde fácilmente obtenemos

$$
L_{c}=\frac{U_{s}^{2}}{2} \frac{\sin \left(2 \varphi_{0}\right)}{\left(a+D_{\Omega}\right)^{2}} .
$$

Así que $\sigma_{12}=2 L_{b}-L_{c}$, y por tanto

$$
\sigma_{12}=-\frac{U_{s}^{2} D_{\Omega} \sin \left(2 \varphi_{0}\right)}{2\left(a+D_{\Omega}\right)^{2}\left(a+2 D_{\Omega}\right)} .
$$

También se cumple que $\sigma_{12}=\sigma_{21}$. 


\section{Apéndice $\mathrm{E}$}

\section{Escalas de tiempo}

En el equilibrio térmico, el promedio de la energía cinética traslacional de las partícula brownianas es el mismo que el de las moléculas del fluido (solvente). Sin embargo, el tamaño y la masa de las partículas brownianas son mucho más grande que el tamaño y masa de las moléculas del fluido. Luego entonces las moléculas se mueven con mucha mayor rapidez que las partículas brownianas.

\begin{tabular}{|c|c|}
\hline Radio de la esfera & $r=100 \mathrm{~nm}$ \\
\hline Densidad de masa de la esfera & $\rho=1.0 \mathrm{~g} \mathrm{~cm}^{-3}$ \\
\hline Temperatura & $298 \mathrm{~K}$ \\
\hline Viscosidad del agua & $\eta=1.0$ centipoise $=10^{-3}$ Pa seg \\
\hline
\end{tabular}

Tabla E.1: Valores estándar de una partícula brownina esférica en agua.

Para poder entender el MB es necesario distinguir ciertas escalas de tiempo para las cuales es válida la ecuación propuesta por Langevin. Estas escalas de tiempo se definen de la siguiente manera:

(i). Tiempo de colisión molecular $\tau_{c}$.

De acuerdo con la teoría cinética molecular, esta escala de tiempo se puede estimar a través de la energía cinética promedio de las moléculas dada por $\left\langle E_{c}\right\rangle=(1 / 2) m\left\langle v^{2}\right\rangle=(3 / 2) k_{B} T$. De esta manera tenemos que $\tau_{c} \sim d / \sqrt{k_{B} T / m}$, donde $d$ es la distancia promedio que recorre la molécula justo antes de colisionar con alguna otra. Si se considera que $d=a$, siendo 
$a$ el radio promedio de una molécula entonces, para un radio típico de $10^{-10}$ $\mathrm{m}$, y como fluido el agua a temperatura normal $T=298 \mathrm{~K}$, obtenemos un tiempo estimado de $\tau_{c} \sim 10^{-13} \mathrm{~s}$. Durante este tiempo, la PB permanece prácticamente en reposo, por lo que $\tau_{c}$ representa también el tiempo de colisión entre la PB con las moléculas del fluido. El inverso de esta escala de tiempo se puede interpretar como la frecuencia de colisión de la moléculas con la $\mathrm{PB}$, que en este caso es del orden de $10^{13}$ colisiones por segundo. Este efecto acumulado de tantas colisiones por segundo hacen que la PB pueda desplazarse de un punto a otro de manera azarosa o irregular. Es evidente que para tiempos de observación $t$ de la $\mathrm{PB}$, del orden de segundos, se cumple que $t \gg \tau_{c}$. En dicha escala de tiempo $t$ la $\mathrm{PB}$ se mueve prácticamente un fluido continuo y no discreto. El movimiento de la partícula browniana se amortigua por la fricción de Stokes y es este amortiguamiento viscoso por el cual la partícula "relaja"su momento.

Por otro lado, si consideramos dos tiempos distintos de observación $t$ y $t^{\prime}$ en la dinámica de la $\mathrm{PB}$, es razonable suponer que para la diferencia de tiempos tales $\left|t-t^{\prime}\right| \gg \tau_{c}$, la función de correlación del ruido $\left\langle\Gamma(t) \Gamma\left(t^{\prime}\right)\right\rangle=0$. Esto significa que las colisiones que experimenta la $\mathrm{PB}$ con las moléculas del fluido al tiempo $t$ no tiene nada que ver con las colisiones que experimenta al tiempo $t^{\prime}$, es decir, son independientes, de manera que en el límite de $\tau_{c} \rightarrow 0$ se tiene que $\left\langle\Gamma(t) \Gamma\left(t^{\prime}\right)\right\rangle=\lambda \delta\left(t-t^{\prime}\right)$. Esta expresión cuantifica la correlación instantánea del ruido de intensidad $\lambda$, o lo que es lo mismo que no tiene memoria.

(ii). Tiempo de relajación del momento lineal $\tau_{m r}$.

La dinámica de la PB también experimenta una fuerza de fricción que produce el amortiguamiento de la partícula y como consecuencia la relajación de su momento lineal $\mathbf{p}=m \mathbf{v}$. De acuerdo con la ley de Stokes, la fuerza de fricción está dada por $f_{s}=-\alpha \mathbf{v}$, donde $\alpha>0$ es el coeficiente de fricción, para el caso de una partícula esférica $\alpha=6 \pi \eta r$, siendo $r$ el radio de la $\mathrm{PB}$. De acuerdo con la segunda ley de Newton $m \frac{d \mathbf{v}}{d t}=-\alpha \mathbf{v}$, y para tiempos de observación $t \gg \tau_{c}$, la velocidad de la $\mathrm{PB}$ decae como $\mathbf{v}(t)=\mathbf{v}_{0} e^{-t / \tau_{m r}}$, donde $\mathbf{v}_{0}$ es la velocidad inicial y $\tau_{m r}=m / \alpha$ el tiempo de relajación. Para esferas en agua el valor de este tiempo de relajación es del orden de $\tau_{m r} \sim 10^{-8} \mathrm{~s}$, que es mucho mayor que el tiempo de colisión, es decir que, $\tau_{c} \ll \tau_{m r}$. Esto justifica la utilización de la fuerza de fricción de Stokes en la dinámica de la PB. 
(iii) Tiempo Browniano o tiempo difusivo $\tau_{B}$.

Cuando la PB ha efectuado muchos cambios en su momento, ésta entra en el régimen de tiempo difusivo $t \gg \tau_{m s}$. Esto significa que el desplazamiento neto de la partícula es independiente de su masa. Para que el MB cambie la posición de las partículas significativamente, tenemos que esperar al menos un tiempo $\tau_{B}$ para que una partícula se difunda una distancia igual a su radio, es decir, $\tau_{B} \sim r^{2} / D_{B}=\eta r^{3} / k_{B} T$ que es del orden de varios segundos. En conclusión, la dinámica de la $\mathrm{PB}$ se lleva a cabo tomando en cuenta la separación de las escalas de tiempo $\tau_{c} \ll \tau_{m r} \ll \tau_{B}$, para los cuales es válida la ecuación de Langevin (4.1).

\section{(iv). Tiempo de relajación del momento angular $\tau_{a r}$}

Para una partícula browniana esférica que rota a una velocidad angular $\boldsymbol{\Omega}$ alrededor de un cierto eje que pasa por su centro de masa, nos preguntamos por el tiempo $\tau_{a r}$ que tarda la esfera en disipar todo su momento angular debido a la viscosidad del fluido. La segunda ley de Newton en este caso está dada por $I \frac{d \boldsymbol{\Omega}}{d t}=-f_{\text {rot }} \boldsymbol{\Omega}$, siendo $I$ el momento de inercia y $f_{\text {rot }}$ es el coeficiente de fricción rotacional dada por $f_{\text {rot }}=8 \pi \eta r^{3}$. En el caso de la partícula esférica homogénea y densidad de masa $\rho$, el momento de inercia está dado por $I=(2 / 5) m r^{2}=(8 / 15) \rho \pi r^{5}$. La solución de la ecuación de Newton en este caso será $\boldsymbol{\Omega}(t)=\boldsymbol{\Omega}_{0} e^{-t / \tau_{a r}}$, donde $\boldsymbol{\Omega}_{0}$ es la velocidad angular al tiempo $t=0$ y $\tau_{a r}$ el tiempo de relajación del momento angular $\mathbf{L}=I \boldsymbol{\Omega}$, tal que $\tau_{a r}=f_{\text {rot }} / I=(\rho / 15 \eta) r^{2}$. Comparando $\tau_{a r}$ con el tiempo de relajación del momento lineal $\tau_{m r}$ se puede concluir que la traslaciones y las rotaciones de la esfera decaen en la misma escala de tiempo. Por lo tanto, en la escala de tiempo difusivo, tanto los momentos traslacionales como los angulares se han relajado por completo. En otras palabras, en el régimen difusivo, la suma de todas las fuerzas y la suma de todas las torcas, ambas son cero.

\section{Tiempo de relajación rotacional $\tau_{r r}$}

Cuando la partícula esférica ha realizado muchos pasos angulares en los que intercambia momento angular con el solvente, ingresa al régimen de tiempo difusivo $t \gg \tau_{a r}$. Inicialmente el desplazamiento angular neto $\theta$ de la esfera es insignificante. Sin embargo, para que $\theta$ se desvíe significativamente 
de su valor inicial, tenemos que esperar al menos un tiempo de relajación

$$
\tau_{r r}=\frac{1}{D_{\Omega}} \sim \frac{\eta r^{3}}{k_{B} T}
$$

Aquí $D_{\Omega}=k_{B} T / 8 \pi \eta r^{3}$ es el coeficiente de difusión rotacional que determina la decaimiento en la orientación de la esfera. Por lo tanto, el tiempo $\tau_{B}$ que le toma a una esfera, para cambiar significativamente su posición coincide con el tiempo que $\tau_{r r}$ necesario para cambiar significativamente su orientación. 


\section{Bibliografía}

[1] J. R. Whitehead. Super-Regenerative Receiver. Cambridge University Press, Cambridge, 1950.

[2] G. Vemuri and R. Roy. Super-regenerative laser receiver: Transient dynamics of a laser with an external signal. Phys. Rev. A, 39:2539, 1989.

[3] Salvador Balle, F. De Pasquale, and M. San Miguel. Passage-time calculation for the detection of weak signals via the transient dynamics of a laser. Phys. Rev. A, 41:5012-5015, May 1990.

[4] Ian Littler, Stefan Balle, Klaas Bergmann, Gautam Vemuri, and Rajarshi Roy. Detection of weak signals via the decay of an unstable state: Initiation of an injection-seeded laser. Phys. Rev. A, 41:41314134, Apr 1990.

[5] J. I. Jiménez Aquino and J. M. Sancho. The decay of unstable states and the quasideterminisc theory. Phys. Rev. A, 43:589, 1991.

[6] H.A. Kramers. Brownian motion in a field of force and the diffusion model of chemical reactions. Physica, 7(4):284 - 304, 1940.

[7] A. L. Pankratov. Time evolution of averages in dynamical systems driven by noise. Phys. Lett. A, 255:17, 1999.

[8] Paul C. Bressloff and Jay M. Newby. Stochastic models of intracellular transport. Rev. Mod. Phys., 85:135-196, Jan 2013.

[9] H. W. McKenzie, M. A. Lewis, and E. H. Merrill. Bulletin of Mathematical Biology, 71:107, 2009. 
[10] R. Metzler, G. Oshanin, and S. Redner. First-Passage Phenomena and Their Applications. World Scientific Publishing Company, Singapore, 2014 .

[11] F. de Pasquale and P. Tombesi. The decay of an unstable equilibrium state near a "critical point". Physics Letters A, 72(1):7 - 9, 1979.

[12] J. I. Jiménez Aquino and Romero-Bastida. Detection of weak and large electric fields through the transient dynamics of a brownian particle in a electromagnetic field. Phys. Rev. E, 81:031128, 2010.

[13] J. I. Jiménez Aquino and Romero-Bastida. Detection of weak signals through nonlinear relaxation times for a brownian particle in a electromagnetic field. Phys. Rev. E, 84:011137, 2011.

[14] H. C. Berg. E. Coli in Motion. Springer-Verlag, New York, 2004.

[15] Paula Watnick and Roberto Kolter. Biofilm, city of microbes. Journal of Bacteriology, 182(10):2675-2679, 2000.

[16] T. Surrey, F. Nédélec, S. Leibler, and E. Karsenti. Science, 292:1167, 2001.

[17] Christian A. Yates, Radek Erban, Carlos Escudero, Iain D. Couzin, Jerome Buhl, Ioannis G. Kevrekidis, Philip K. Maini, and David J. T. Sumpter. Inherent noise can facilitate coherence in collective swarm motion. Proceedings of the National Academy of Sciences, 106(14):5464-5469, 2009.

[18] T. Sanchez, D. T. N. Chen, S. J. DeCamp, M. Heymann, and Z. Dogic. Nature, 491:431, 2012.

[19] Yu. M. Romanovsky, A. V. Kargovsky, and W. Ebeling. Eur. Phys. J. Special Topics, 222:2465, 2013.

[20] Ramin Golestanian, Tanniemola B. Liverpool, and Armand Ajdari. Propulsion of a molecular machine by asymmetric distribution of reaction products. Phys. Rev. Lett., 94:220801, Jun 2005. 
[21] Hong-Ren Jiang, Natsuhiko Yoshinaga, and Masaki Sano. Active motion of a janus particle by self-thermophoresis in a defocused laser beam. Phys. Rev. Lett., 105:268302, Dec 2010.

[22] D. G. Crowdy. J. Fluid Mech., 735:473, 2013.

[23] Xu Zheng, Borge ten Hagen, Andreas Kaiser, Meiling Wu, Haihang Cui, Zhanhua Silber-Li, and Hartmut Löwen. Non-gaussian statistics for the motion of self-propelled janus particles: Experiment versus theory. Phys. Rev. E, 88:032304, Sep 2013.

[24] D. Bray. Cell Movements. Garland, New York, 2000.

[25] Luca Angelani. 48:495003, 2015.

[26] Enrique Puga Cital. Difusión Efectiva en Sistemas de Materia Activa Diluida. Tesis de Maestría, UNAM 2017.

[27] G. Volpe F. Kümmel G. Volpe I. Buttinoni and Clemens Bechinger. Active brownian motion tunable by light. Journal Phys. Condens Matter, 24:284129, 2012.

[28] E. Lauga and T. R. Powers. The hydrodynamics of swimming microorganisms. Rep. Prog. Phys, 72:096601, 2009.

[29] E. M. Purcell. Life at low reynolds number. American Journal of Physics, 45(1):3-11, 1977.

[30] Robert Brown. A brief account of microscopical observations made in the months of june, july, and august, 1827, on the particles contained in the pollen of plants; and on the general existence of active molecules in organic and inorganic bodies. Philos. Mag., 4:161-173, 1828.

[31] Albert Einstein. On the motion of small particles suspended in liquids at rest required by the molecular-kinetic theory of heat. Annalen der Physik, 17(8):549-560, 1905.

[32] Marian Smoluchowski. Outline of the kinetic theory of brownian motion of suspensions. Annalen der Physik, 21:756, 1906. 
[33] P. Langevin. Paul langevin's 1908 paper "on the theory of brownian motion" ["sur la théorie du mouvement brownien," c. r. acad. sci. (paris) 146, 530-533 (1908)]. Am. J. of Phys., 65:1079-1081 (1997), June 1997.

[34] J. B. Perrin. "mouvement brownien et réalité moléculaire", ann. chim. phys. 18, 5-114 (1909), j. perrin, atoms, translated by d. ll. hammick, (constable, london, 1920) and reprinted by (ox bow, woodbridge, 1990), chaps. ii-iv.

[35] H. Risken. The Fokker-Planck Equation. Springer, Berlin Heidelberg, 1996.

[36] Hannes Risken and Till Frank. The Fokker-Planck Equation : Methods of Solution and Applications, volume 18. Springer-Verlag Berlin Heidelberg, 2 edition, 1984.

[37] G. E. Uhlenbeck and L. S. Ornstein. On the theory of the brownian motion. Phys. Rev., 36:823-841, Sep 1930.

[38] Lefever R. Horsthemke, W. Noise-Induced Transitions Theory and Applications in Physics, Chemistry, and Biology. Springer-Verlag Berlin.

[39] J. Dellunde, M. C. Torrent, and J. M. Sancho. Opt. Commun., 102:277, 1993.

[40] J. I. Jiménez-Aquino and M. Romero-Bastida. Rotating unstable langevin-type dynamics: Linear and nonlinear mean passage time distributions. Phys. Rev. E, 66:061101, Dec 2002.

[41] M. Abramowitz and I. A. Stegun. Handbook of Mathematical Functions. Dover, New York, 1972.

[42] Francisco J. Sevilla and Mario Sandoval. Smoluchowski diffusion equation for active brownian swimmers. Phys. Rev. E, 91:052150, May 2015 .

[43] Mario Sandoval, Navaneeth K. Marath, Ganesh Subramanian, and Eric Lauga. Stochastic dynamics of active swimmers in linear flows. Journal of Fluid Mechanics, 742:50-70, 2014. 
[44] Pawel Romanczuk, Markus Bär, Werner Ebeling, Benjamin Lindner, and Lutz Schimansky-Geier. Active brownian particles. from individual to collective stochastic dynamics. The European Physical Journal Special Topics, 202:1-162, 022012.

[45] J T Waldron William Coffey, Yu P Kalmykov. The Langevin equation: with applications in physics, chemistry, and electrical engineering. Singapore : World Scientific, 1996.

[46] E. piña. Dinámica de rotaciones. Universidad Autónoma Metropolitana, 1996.

[47] E. piña. Acta mexicana de ciencia y tecnología. VII:55, 1973. 
PARTICULAS ACTIVAS EN LA DETECCIÓN DE SEÑALES DÉBILES.

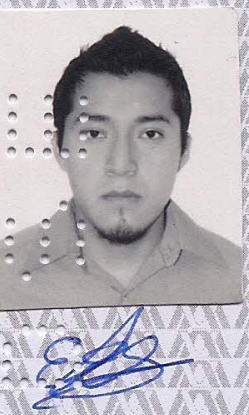

ERNESTO JOEL ESPINOSA SANTAMARIA ALUMNO

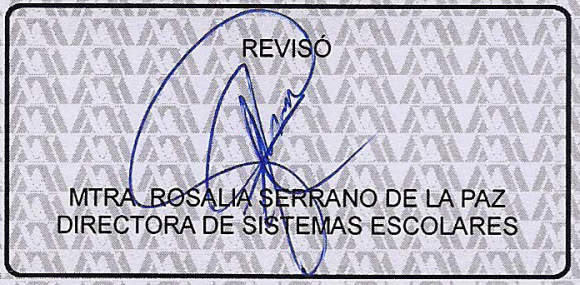

DIRECTOR DE LA DIVISIÓN DE CB

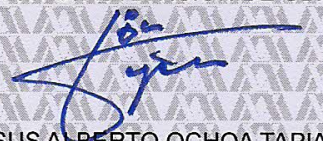

DR JESUSA BERTO OCHOATAPIA

Acto continuo, el presidente del jurado comunicó al interesado el resultado de la evaluación y, en caso aprobatorio, le fue tomada la protesta.

En la Ciudad de México, se presentaron a las 11:00 horas del día 6 del mes de diciembre del año 2019 en la Unidad Iztapalapa de la Universidad Autónoma Metropolitana, los suscritos miembros del jurado:

DR. ROSALIO FERNANDO RODRIGUEZ ZEPEDA

DR. FRANCISCO JAVIER SEVILLA

DR. JOSE INES JIMENEZ AQUINO

Bajo la Presidencia del primero y con carácter de Secretario el último, se reunieron para proceder al Examen de Grado cuya denominación aparece al margen, paralla obtención del grado de:

MAESTRO EN CIENCIAS (FISICA)

DE: ERNESTO JOEL ESPINOSA SANTAMARIA

y de acuerdo con el articulo 78 fracción III del Reglamento de Estudios Superiores de la Universidad Autónoma Metropolitana, los miembros del jurado resolvieron:

\section{ARIRIOBAR}

VOCAL

tramess. Lum sortla

DR. FRANCISCO JAVIER SEVILLA
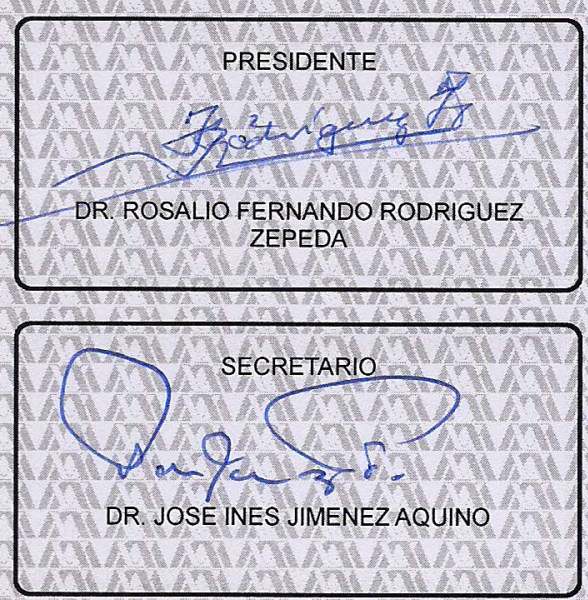